\title{
APPROXIMATION FORTE POUR LES VARIÉTÉS AVEC UNE ACTION D'UN GROUPE LINÉAIRE
}

\author{
YANG CAO
}

\begin{abstract}
RÉSUMÉ. Soit $G$ un groupe linéaire connexe sur un corps de nombres. Soit $U \hookrightarrow X$ une inclusion $G$-équivariante d'un $G$-espace homogène à stabilisateurs connexes dans une $G$-variété lisse. On montre que $X$ satisfait l'approximation forte avec condition de Brauer-Manin hors d'un ensemble $S$ de places de $k$ dans chacun des cas suivants :

(i) $S$ est l'ensemble des places archimédiennes;

(ii) $S$ est un ensemble fini non vide quelconque, et $\bar{k}^{\times}=\bar{k}[X]^{\times}$.

La démonstration utilise le cas $X=U$, qui a fait l'objet de divers travaux.

Summary. Let $G$ be a connected linear algebraic group over a number field. Let $U \hookrightarrow X$ be a $G$-equivariant open embedding of a $G$-homogeneous space with connected stabilizers into a smooth $G$-variety. We prove that $X$ satisfies strong approximation with Brauer-Manin condition off a set $S$ of places of $k$ under either of the following hypotheses :

(i) $S$ is the set of archimedean places;

(ii) $S$ is a nonempty finite set and $\bar{k}^{\times}=\bar{k}[X]^{\times}$.

The proof builds upon the case $X=U$, which has been the object of several works.
\end{abstract}

\section{TABLE DES MATIÈRES}

1. Introduction

2. Préliminaires sur les $G$-variétés et les torseurs 5

2.1. Préliminaires sur les $G$-variétés 6

2.2. Trivialisation d'un torseur 8

2.3. L'action d'un groupe sur un torseur 10

3. Groupe de Brauer invariant 12

3.1. Définitions et propriétés 13

3.2. L'homomorphisme de Sansuc 16

3.3. Pseudo espace homogène 19

4. L'approximation forte hors des places archimédiennes et la question $1.2 \quad 21$

5. La descente par rapport au groupe de Brauer invariant 23

5.1. Torseur sous un groupe linéaire dont le groupe de Shafarevich est trivial 23

5.2. L'application de la résolution coflasque 24

5.3. La descente générale 26

6. La méthode de fibration et la question $1.3 \quad 28$

6.1. L'approximation forte raffinée pour l'espace affine 29

6.2. Fibration sur une variété torique standard 31

6.3. Fibration sur un tore 34

Date: 8 novembre 2018. 
6.4. Fibration sur un pseudo espace homogène

\section{INTRODUCTION}

Soit $k$ un corps de nombres. On note $\Omega_{k}$ l'ensemble des places de $k$ et $\infty_{k}$ l'ensemble des places archimédiennes de $k$. On note $v<\infty_{k}$ pour $v \in \Omega_{k} \backslash \infty_{k}$. Notons $\mathcal{O}_{k}$ l'anneau des entiers de $k$ et $\mathcal{O}_{S}$ l'anneau des $S$-entiers de $k$ pour un sous-ensemble fini $S$ de $\Omega_{k}$ contenant $\infty_{k}$. Pour chaque $v \in \Omega_{k}$, on note $k_{v}$ le complété de $k$ en $v$ et $\mathcal{O}_{v} \subset k_{v}$ l'anneau des entiers $\left(\mathcal{O}_{v}=k_{v}\right.$ pour $v \in \infty_{k}$ ). Soit $\mathbf{A}_{k}$ l'anneau des adèles de $k$. Pour un sous-ensemble fini $S \subset \Omega_{k}$, soit $\mathbf{A}_{k}^{S}$ l'anneau des adèles hors de $S$ et $k_{S}:=\prod_{v \in S} k_{v}$. Par exemple, soit $\mathbf{A}_{k}^{\infty}$ l'anneau des adèles finis et $k_{\infty}:=\prod_{v \in \infty_{k}} k_{v}$.

On rappelle les définitions de [CTX09], [CTX13, §2], [BD] et [CX1].

Pour $B$ sous-ensemble de $\operatorname{Br}(X)$, on définit

$$
X\left(\mathbf{A}_{k}\right)^{B}=\left\{\left(x_{v}\right)_{v \in \Omega_{k}} \in X\left(\mathbf{A}_{k}\right): \sum_{v \in \Omega_{k}} i n v_{v}\left(\xi\left(x_{v}\right)\right)=0 \in \mathbb{Q} / \mathbb{Z}, \quad \forall \xi \in B\right\} .
$$

Comme l'a remarqué Manin, la théorie du corps de classes donne $X(k) \subseteq X\left(\mathbf{A}_{k}\right)^{B}$.

Définissons

$$
X\left(\mathbf{A}_{k}\right) \bullet=\pi_{0}\left(X\left(k_{\infty}\right)\right) \times X\left(\mathbf{A}_{k}^{\infty}\right)
$$

la projection, où $\pi_{0}\left(X\left(k_{\infty}\right)\right)$ est l'ensemble des composantes connexes de $X\left(k_{\infty}\right)$.

Puisque, pour tout $v \in \infty_{k}$, chaque élément de $\operatorname{Br}(X)$ prend une valeur constante sur chaque composante connexe de $\pi_{0}\left(X\left(k_{v}\right)\right)$, pour tout sous-ensemble $B \subset \operatorname{Br}(X)$ on peut définir :

$$
X\left(\mathbf{A}_{k}\right)_{\bullet}^{B}=\left\{\left(x_{v}\right)_{v \in \Omega_{k}} \in X\left(\mathbf{A}_{k}\right)_{\bullet}: \sum_{v \in \Omega_{k}} i n v_{v}\left(\xi\left(x_{v}\right)\right)=0 \in \mathbb{Q} / \mathbb{Z}, \quad \forall \xi \in B\right\} .
$$

Définition 1.1. ([CTX09, CTX13]) Soient $k$ un corps de nombres, $X$ une $k$-variété et $S \subset \Omega_{k}$ un sous-ensemble fini. Notons $\operatorname{Pr}^{S}: X\left(\mathbf{A}_{k}\right) \rightarrow X\left(\mathbf{A}_{k}^{S}\right)$ la projection. $S$.

(1) Si $X(k)$ est dense dans $\operatorname{Pr}^{S}\left(X\left(\mathbf{A}_{k}\right)\right)$, on dit que $X$ satisfait l'approximation forte hors de

(2) Si $X(k)$ est dense dans $\operatorname{Pr}^{S}\left(X\left(\mathbf{A}_{k}\right)^{B}\right)$ pour un sous-ensemble $B$ de $\operatorname{Br}(X)$, on dit que $X$ satisfait l'approximation forte par rapport à $B$ hors de $S$. On dit aussi alors que $X$ satisfait l'approximation forte de Brauer-Manin par rapport à $B$ hors de $S$.

Si $S=\infty_{k}$, on peut s'intéresser à une question un peu plus précise tenant compte des composantes connexes réelles : $X(k)$ est-il dense dans $X\left(\mathbf{A}_{k}\right)_{\bullet}^{B}$ pour un sous-groupe $B \subset \operatorname{Br}(X)$ ?

Pour les espaces homogènes de groupes algébriques connexes, ces questions ont fait ces dernières années l'objet d'une série de travaux [CTX09, Ha08, BD] prolongeant des travaux classiques.

Lorsqu'on cherche à étendre la classe des variétés satisfaisant les propriétés ci-dessus, il est naturel de poser les questions 1.2 et 1.3 suivantes. 
Question 1.2. Soient $X$ une $k$-variété lisse géométriquement intègre et $U$ un ouvert de $X$. Si $U(k)$ est dense dans $U\left(\mathbf{A}_{k}\right)_{\bullet}^{\operatorname{Br}(U)}$, sous quelles conditions peut-on établir que $X(k)$ est dense dans $X\left(\mathbf{A}_{k}\right)_{\bullet}^{\operatorname{Br}(X)}$ ?

Le cas qui nous intéresse est celui où $U$ est un $G$-espace homogène. En général, l'inclusion $\bar{k}^{\times} \subset \bar{k}[U]^{\times}$n'est pas un isomorphisme et, dans ce cas, il existe des exemples pour lesquels $U$ ne satisfait pas l'approximation forte par rapport à $\operatorname{Br}(U)$ hors d'un sous-ensemble fini $S \subset \Omega_{k}$ non vide quelconque (par exemple $U \cong \mathbb{G}_{m}, k=\mathbb{Q}$ et $S=\left\{v_{0}\right\}$ avec $v_{0}$ une place non archimédienne).

Question 1.3. Soit $S \subset \Omega_{k}$ un sous-ensemble fini non vide. Soient $X$ une $k$-variété lisse géométriquement intègre et $U$ un ouvert de $X$ fibré sur un certain $G$-espace homogène $Z$. Si toute fibre de $U$ au-dessus d'un $k$-point de $Z$ satisfait l'approximation forte hors de $S$, sous quelles conditions peut-on établir l'approximation forte pour $X$ par rapport à $\operatorname{Br}(X)$ hors de $S$ ?

Le principal résultat de cet article est le :

Théorème 1.4. (cf. Théorème \%.6) Soit $G$ un groupe linéaire connexe sur un corps de nombres $k$, et soit $X$ une $G$-variété lisse géométriquement intègre sur $k$. Supposons qu'il existe un $G$ ouvert $U \subset X$ tel que $U \cong G / G_{0}$, où $G_{0} \subset G$ est un sous-groupe fermé connexe. Soit $S \subset \Omega_{k}$ un sous-ensemble fini non vide et supposons que $G^{\prime}\left(k_{S}\right)$ est non compact pour chaque facteur simple $G^{\prime}$ du groupe $G^{s c}$.

(1) Si $S \subset \infty_{k}$, alors $X(k)$ est dense dans $X\left(\mathbf{A}_{k}\right)_{\bullet}^{\operatorname{Br}(X)}$.

(2) Si $\bar{k}^{\times}=\bar{k}[X]^{\times}$, alors $X$ satisfait l'approximation forte de Brauer-Manin par rapport $\grave{a}$ $\operatorname{Br}(X)$ hors de $S$.

Ce théorème est une conséquence directe d'un résultat plus général mais d'énoncé technique (Théorème 7.5).

Le théorème 1.4 (1) avait déjà été établi dans les cas suivants :

(i) $X$ est une variété torique, c'est-à-dire que $G$ est un tore (F. Xu et l'auteur $[\mathrm{CX}]$ ).

(ii) $X$ est une variété groupique, c'est-à-dire que $G$ est un groupe linéaire connexe et que $G_{0}=1$ (F. Xu et l'auteur [CX1]).

(iii) $X$ est comme dans le théorème, avec $G_{0}$ résoluble connexe (résultat tout récent de $\mathrm{D}$. Wei [Wei16]).

$\mathrm{Au} \S 4$, on donne une démonstration totalement nouvelle de ce résultat, laquelle est plus simple que celles des trois travaux ci-dessus.

Le théorème 1.4 (2) avait déjà été établi dans les cas suivants :

(iv) $X$ est une variété torique (D. Wei [Wei14]).

(v) $X$ est une variété groupique (F. Xu et l'auteur [CX1]).

Les démonstrations de ce résultat, comme celle de [CX1], reposent d'une part sur des constructions géométriques sur un corps quelconque, d'autre part sur les théorèmes d'approximation forte avec condition de Brauer-Manin pour les espaces homogènes établis dans [CTX09, Ha08, $\mathrm{BD}$. Les constructions géométriques du présent article s'inspirent de celles de [CX1] et la partie arithmétique du présent article utilise une généralisation de [CDX] (cf. §5).

Dans les quatre articles cités, on a la conclusion plus précise : dans les énoncés, on peut remplacer $\operatorname{Br}(X)$ par le sous-groupe "algébrique" $\operatorname{Br}_{1}(X) \subset \operatorname{Br}(X)$, dont la définition est rappelée ci-dessous. Dans le cadre général où nous nous plaçons, il faut utiliser tout le groupe de Brauer 
$\operatorname{Br}(X)$. L'idée clé est la notion de sous-groupe de Brauer invariant (cf. §3), qui généralise [BD, $\S 1.2]$.

Soit $G$ un groupe linéaire connexe. Le plan de l'article est le suivant :

La section 2 est consacrée à divers préliminaires géométriques, notamment sur les $G$-variétés et leurs torseurs sous un tore ou un groupe de type multiplicatif. Au §2.3, on étudie les torseurs sous un groupe de type multiplicatif sur une $G$-variété. On montre que tout tel torseur peut être muni canoniquement d'une action d'un groupe linéaire $H$ qui s'envoie sur $G$ (Théorème 2.7). Pour cela, on utilise un théorème de Colliot-Thélène [CT08, Thm. 5.6] sur les torseurs au-dessus d'un groupe linéaire connexe.

$\mathrm{Au} \S 3$, on définit la notion du sous-groupe de Brauer $G$-invariant $\operatorname{Br}_{G}(X)$ d'une $G$-variété lisse (cf. Définition 3.1). On donne des caractérisations équivalentes sur les sous-groupes de $\operatorname{Br}_{G}(X)$ (Proposition 3.3). Ensuite, on définit la notion d'homomorphisme de Sansuc (cf. Définition 3.8) et on généralise la suite exacte de Sansuc (cf. (3.6)). On étudie la propriété de $\operatorname{Br}_{G}(X)$ par rapport au passage à la fibre d'un $G$-morphisme vers un tore (Proposition 3.13). On généralise la notion de $G$-espace homogène à stabilisateur géométrique connexe et on définit la notion de pseudo $G$-espace homogène (Définition 3.15) qui sera utilisée aux $\S 4$ et $\S 6$.

La section 4 est consacrée à l'approximation forte hors des places archimédiennes. On établit le théorème 4.2 sur l'approximation d'un point adélique de $X$ satisfaisant une condition de Brauer-Manin par un tel point situé sur $U$. Comme conséquence, on répond à la question 1.2 (Corollaire 4.5) dans ce cas. Ceci donne le théorème 1.4 (1).

$\mathrm{Au} \S 5$, en utilisant la notion de sous-groupe $G$-invariant du groupe de Brauer, on combine la suite exacte (3.6) et la méthode de [CDX], et on généralise [CDX, Thm. 1.2]. On établit un théorème de descente pour un torseur sous un groupe linéaire connexe quelconque (Théorème 5.9). Comme conséquence, on établit une formule sur les points adéliques d'un certain $G$-espace homogène satisfaisant une condition de Brauer-Manin (Corollaire 5.13).

$\mathrm{Au} \S 6$, pour une $G$-variété $X$ munie d'un $G$-ouvert $U$ fibré sur l'autre $G$-variété $Z$, on considère l'approximation d'un point adélique de $X$ satisfaisant une condition de Brauer-Manin par un tel point situé dans la fibre au-dessus d'un point rationnel de $Z$ (Question 6.1). Pour répondre à la question 6.1, on généralise [CX1, Prop. 3.6] et on établit au $\S 6.1$ un théorème plus fin sur l'approximation forte d'un espace affine (théorème 6.2). Ensuite, on combine ce théorème et l'étude du sous-groupe de Brauer $G$-invariant (§3) avec la méthode de fibration de ColliotThélène et Harari [CTH], et on répond à la question 6.1 dans le cas où $Z$ est un certain tore (Théorème 6.7 et Théorème 6.9). Au cas où $Z$ est un pseudo $G$-espace homogène, on résoud la question 6.1 (Théorème 6.11) à l'aide de tous les résultats ci-dessus (sauf ceux du §4).

La section 7 est consacrée à la preuve des résultats principaux à l'aide de tous les résultats ci-dessus. En utilisant la descente (§5), on établit d'abord un résultat (Proposition 7.4) sur l'approximation forte pour les $G$-espaces homogènes à stabilisateur géométrique connexe, ce qui généralise un résultat de Borovoi et Demarche ([BD, Thm. 1.4]). Ensuite, on combine ce résultat avec les résultats des $\S 4$ et $\S 6$, et on établit le théorème principal (Théorème 7.6 ) sur l'approximation forte d'une $G$-variété munie d'un $G$-ouvert fibré sur un certain $G$-espace homogène. Ceci donne immédiatement le théorème 1.4.

\section{Conventions et notations.}


Soit $k$ un corps quelconque de caractéristique 0 . On note $\bar{k}$ une clôture algébrique et $\Gamma_{k}:=$ $\operatorname{Gal}(\bar{k} / k)$.

Tous les groupes de cohomologie sont des groupes de cohomologie étale. Soit $X$ un schéma intègre. On note $\eta_{X}$ le point générique de $X$.

Une $k$-variété $X$ est un $k$-schéma séparé de type fini. Pour $X$ une telle variété, on note $k[X]$ son anneau des fonctions globales, $k[X]^{\times}$son groupe des fonctions inversibles, $\operatorname{Pic}(X):=$ $H_{\text {êt }}^{1}\left(X, \mathbb{G}_{m}\right)$ son groupe de Picard et $\operatorname{Br}(X):=H_{\text {èt }}^{2}\left(X, \mathbb{G}_{m}\right)$ son groupe de Brauer. Notons

$$
\operatorname{Br}_{1}(X):=\operatorname{Ker}\left[\operatorname{Br}(X) \rightarrow \operatorname{Br}\left(X_{\bar{k}}\right)\right] \text { et } \operatorname{Br}_{a}(X):=\operatorname{Br}_{1}(X) / \operatorname{ImBr}(k) \text {. }
$$

Le groupe $\mathrm{Br}_{1}(X)$ est le sous-groupe "algébrique" du groupe de Brauer de $X$. Si $X$ est intègre, on note $k(X)$ son corps des fonctions rationnelles.

On note $X_{\text {sing }}$ le lieu singulier de $X$, et pour un sous-ensemble fermé $D \subset X$, on note $D_{\text {sing }}$ le lieu singulier de $D$ comme sous-variété fermée réduite.

Un $k$-groupe algébrique $G$ est une $k$-variété qui est un $k$-schéma en groupes. On note $e_{G}$ l'unité de $G$ et $G^{*}$ le groupe des caractères de $G_{\bar{k}}$. C'est un module galoisien de type fini. Si $G$ est connexe, on note

$$
\operatorname{Br}_{e}(G):=\operatorname{Ker}\left(\operatorname{Br}_{1}(G) \stackrel{e_{G}^{*}}{\longrightarrow} \operatorname{Br}(k)\right) \cong \operatorname{Br}_{a}(G) .
$$

Soit $G$ un groupe linéaire connexe. On note $G^{\text {tor }}$ son quotient torique maximal, $G^{u}$ son radical unipotent, $G^{\text {red }}:=G / G^{u}$ son quotient réductif maximal, $G^{\text {ssu }}:=\operatorname{Ker}\left(G \rightarrow G^{\text {tor }}\right)$, $G^{s s}:=G^{s s u} / G^{u}$ et $G^{s c}$ le revêtement simplement connexe du groupe semi-simple $G^{s s}$. Alors on a $G^{*}=\left(G^{t o r}\right)^{*}$.

Soit $G$ un $k$-groupe algébrique. Une $G$-variété $(X, \rho)$ (ou $X$ ) est une $k$-variété $X$ munie d'une action à gauche $G \times_{k} X \stackrel{\rho}{\rightarrow} X$. Un ouvert $U \subset X$ est un $G$-ouvert si $U$ est invariant sous l'action de $G$. Un $k$-morphisme de $G$-variétés est appelé $G$-morphisme s'il est compatible avec l'action de $G$.

Soit $G$ un $k$-groupe algébrique connexe et $X$ une $G$-variété lisse géométriquement intègre. Notons $\operatorname{Br}_{G}(X)$ le sous-groupe de Brauer G-invariant (cf : Définition 3.1). Dans le cas où $X \cong G / G_{0}$ avec $G$ linéaire et $G_{0} \subset G$ un sous-groupe fermé connexe, Borovoi et Demarche ont défini $\operatorname{Br}_{1}(X, G):=\operatorname{Ker}\left(\operatorname{Br}(X) \rightarrow \operatorname{Br}\left(G_{\bar{k}}\right)\right)([\mathrm{BD}, \S 1.2])$ En fait, on a (Proposition 3.9) : $\operatorname{Br}_{G}(X) \cong \operatorname{Br}_{1}(X, G)$.

Soit $T$ un tore. Une variété torique $(T \hookrightarrow X)$ est une $T$-variété lisse intègre $X$ munie d'une immersion ouverte fixée $T \hookrightarrow X$ de $T$-variétés. Pour une $k$-algèbre finie séparable $K$, on a une variété torique canonique : $\left(\operatorname{Res}_{K / k} \mathbb{G}_{m} \hookrightarrow \operatorname{Res}_{K / k} \mathbb{A}^{1}\right)$.

Soit $k$ un corps de nombres. Soit $X$ une $k$-variété. On note $X\left(\mathbf{A}_{k}\right)$ l'ensemble des points adéliques de $X$ et on note $X\left(\mathbf{A}_{k}\right)$. comme en (1.1). Soit $G$ un $k$-groupe algébrique. On note $G\left(k_{\infty}\right)^{+}$la composante connexe de l'identité de $G\left(k_{\infty}\right):=\prod_{v \in \infty_{k}} G\left(k_{v}\right)$.

\section{Préliminaires Sur les $G$-VARiétés et les torseurs}

Dans toute cette section, $k$ est un corps quelconque de caractéristique 0. Sauf mention explicite du contraire, une variété est une $k$-variété. Dans [CX1], le résultat de structure géométrique est [CX1, Prop. 3.12]. On en donne une généralisation (Proposition 2.2 et Proposition 2.3). Dans [CTSb, Lem. 1.6.2], Colliot-Thélène et Sansuc ont étudié la structure des torseurs $Y \rightarrow X$ sous un tore et obtenu la suite exacte (2.2). On précise ici les morphismes de la suite exacte (2.2) (Proposition 2.5). Colliot-Thélène ([CT08, Thm. 5.6]) a étudié la structure des torseurs $Y \rightarrow X$ 
sous un groupe de type mutiplicatif lorque la base $X$ est un groupe linéraire connexe. On considère ici le cas plus général des torseurs sur une variété $X$ munie d'une action d'un groupe $G$. On établit un théorème général (Théorème 2.7) sur la structure de ces torseurs.

2.1. Préliminaires sur les $G$-variétés. On rappelle un résultat pour les variétés toriques lisses ([CX, Prop. 2.10]).

Lemme 2.1. Soient $T$ un tore et $(T \hookrightarrow Z)$ une variété torique lisse. Soit $Z_{1}:=Z \backslash\left[(Z \backslash T)_{\text {sing }}\right]$. Alors $\left(T \hookrightarrow Z_{1}\right)$ est une variété torique, $\operatorname{codim}\left(Z \backslash Z_{1}, Z\right) \geq 2$ et chaque $T_{\bar{k}}$-orbite de $\left(Z_{1} \backslash T\right)_{\bar{k}}$ est de codimension 1 et son stabilisateur géométrique est isomorphe $\grave{a} \mathbb{G}_{m, \bar{k}}$.

Démonstration. Puisque $(Z \backslash T)_{\text {sing }}$ est $T$-invariant et $\operatorname{dim}\left((Z \backslash T)_{\text {sing }}\right)<\operatorname{dim}(Z \backslash T)$, la variété $\left(T \hookrightarrow Z_{1}\right)$ est une variété torique et $\operatorname{codim}\left(Z \backslash Z_{1}, Z\right) \geq 2$.

Supposons que $k=\bar{k}$. Dans ce cas, toutes les variétés toriques lisses de dimension $d$ ont un recouvrement par des variétés toriques $\left(\mathbb{G}_{m}^{n} \hookrightarrow \mathbb{G}_{m}^{i} \times \mathbb{A}^{n-i}\right)_{i}$ (cf. [CX, Lem. 2.1]), et donc $Z_{1}$ admet un recouvrement par des variétés toriques $\mathbb{G}_{m}^{\operatorname{dim}(Z)-1} \times \mathbb{A}^{1}$ et $\mathbb{G}_{m}^{\operatorname{dim}(Z)}$. Le résultat en découle.

Proposition 2.2. Soient $T \subset Z_{1} \subset Z$ comme dans le lemme 2.1. Soit $G$ un groupe linéaire connexe muni d'un homomorphisme surjectif $G \stackrel{\varphi}{\rightarrow} T$ de noyau connexe. Soit $X$ une $G$-variété lisse géométriquement intègre munie d'un $G$-morphisme dominant $X \stackrel{f}{\rightarrow} Z$. Soit $U$ un $G$-ouvert de $X$ tel que $f(U) \subset T$. On a :

(1) le morphisme $f^{-1}\left(Z_{1}\right) \stackrel{\left.f\right|_{1}}{\longrightarrow} Z_{1}$ est plat;

(2) si f induit un isomorphisme $\operatorname{Div}_{Z_{\bar{k}} \backslash T_{\bar{k}}}\left(Z_{\bar{k}}\right) \stackrel{f_{\mathrm{Div}}^{*}}{\longrightarrow} \operatorname{Div}_{X_{\bar{k}} \backslash U_{\bar{k}}}\left(X_{\bar{k}}\right)$, alors il existe un $G$-ouvert $X_{1}$ de $X$ tel que $f\left(X_{1}\right) \subset Z_{1}, X_{1} \cap f^{-1}(T)=U, \operatorname{codim}\left(X \backslash X_{1}, X\right) \geq 2$ et $X_{1} \stackrel{\left.f\right|_{1}}{\longrightarrow} Z_{1}$ soit lisse, surjectif à fibres géométriquement intègres.

Démonstration. Par le lemme 2.1, $Z_{1}$ est $T$-invariant, $\operatorname{codim}\left(Z \backslash Z_{1}, Z\right) \geq 2$ et $\left(Z_{1} \backslash T\right)_{\bar{k}}=\coprod_{i} F_{i}$, où $F_{i}$ est $T_{\bar{k}}$-orbite de codimension 1 . Donc $f^{-1}\left(Z_{1}\right)$ et $\overline{f^{-1}(T) \backslash U} \subset X$ sont $G$-invariants.

Pour (1), on peut supposer que $X=f^{-1}\left(Z_{1}\right)$ et $k=\bar{k}$. Puisque $Z_{1}$ est lisse, $X$ est intègre et $f$ est dominant, il existe un ouvert $V \subset Z_{1}$ tel que $\operatorname{codim}\left(Z_{1} \backslash V, Z_{1}\right) \geq 2$ et $f^{-1}(V) \rightarrow V$ soit plat. Donc pour chaque $i, V \cap F_{i} \neq \emptyset$. En utilisant l'action de $G$, on a que $\left.f\right|_{Z_{1}}$ est plat.

Pour (2), puisque $f_{\text {Div }}^{*}$ est un isomorphisme, on a

$$
\operatorname{codim}\left(\overline{f^{-1}(T) \backslash U}, X\right) \geq 2 \text { et } \operatorname{codim}\left(f^{-1}\left(Z \backslash Z_{1}\right), X\right) \geq 2 .
$$

On note $X_{2}:=f^{-1}\left(Z_{1}\right) \backslash \overline{f^{-1}(T) \backslash U}$ et $X_{3}:=X_{2} \backslash\left(X_{2} \backslash U\right)_{\text {sing. }}$ Alors $\left(X_{3} \backslash U\right)=D \coprod E$ avec $\operatorname{codim}\left(E, X_{3}\right) \geq 2$ et toutes les composantes connexes de $D$ sont de dimension $\operatorname{dim}(X)-1$. On note $X_{1}:=X_{3} \backslash E$. Alors $X_{1}$ est $G$-invariant, $f\left(X_{1}\right) \subset Z_{1}, X_{1} \cap f^{-1}(T)=U, \operatorname{codim}\left(X \backslash X_{1}, X\right) \geq$ 2 et chaque composante connexe de $\left(X_{1} \backslash U\right)_{\bar{k}}$ est lisse, intègre de dimension $\operatorname{dim}(X)-1$.

Puisque $f_{\text {Div }}^{*}$ est un isomorphisme, $\left(X_{1} \backslash U\right)_{\bar{k}} \cong \coprod_{i} D_{i}$ avec $D_{i}=f^{-1}\left(F_{i}\right) \cap X_{1}$. Alors chaque $D_{i}$ est une $G_{\bar{k}^{-}}$-variété lisse intègre de dimension $\operatorname{dim}(X)-1$. Puisque $\operatorname{Ker}(\varphi)$ est connexe, le stabilisateur de $F_{i}$ comme $G_{\bar{k}}$-variété est connexe. Par [CX1, Prop. 2.2], les morphismes $U \rightarrow T$ et $D_{i} \rightarrow F_{i}$ sont lisses surjectifs à fibres géométriquement intègres. Le résultat en découle.

Proposition 2.3. Soient $T$ et $T_{0}$ deux tores avec $T_{0}$ quasi-trivial. Soient $X$ une variété lisse géométriquement intègre et $U \subset X$ un ouvert muni d'un morphisme $U \stackrel{f}{\rightarrow} T_{0} \times T$. Supposons 
que la composition

$$
\Lambda: T_{0}^{*} \stackrel{p_{1}^{*}}{\longrightarrow} T_{0}^{*} \times T^{*} \stackrel{f^{*}}{\longrightarrow} \bar{k}[U]^{\times} / \bar{k}^{\times} \stackrel{\operatorname{div}_{X}}{\longrightarrow} \operatorname{Div}_{X_{\bar{k}} \backslash U_{\bar{k}}}\left(X_{\bar{k}}\right)
$$

est un isomorphisme.

(1) Alors il existe un homomorphisme $T_{0} \stackrel{\phi}{\rightarrow} T$ et une variété torique $\left(T_{0} \hookrightarrow \mathbb{A}^{l}\right)$ tels que :

(a) il existe une $k$-algèbre finie séparable $K$ et un isomorphisme de variétés toriques :

$$
\left(T_{0} \hookrightarrow \mathbb{A}^{l}\right) \cong\left(\operatorname{Res}_{K / k} \mathbb{G}_{m} \hookrightarrow \operatorname{Res}_{K / k} \mathbb{A}_{K}^{1}\right) ;
$$

(b) si l'on note

$$
T_{0} \times T \stackrel{\widetilde{\phi}}{\rightarrow} T_{0} \times T:\left(t_{0}, t\right) \mapsto\left(t_{0}, t-\phi\left(t_{0}\right)\right),
$$

alors le morphisme $\widetilde{\phi} \circ f$ peut être étendu à un unique morphisme $X \stackrel{f^{\prime}}{\rightarrow} \mathbb{A}^{l} \times T$;

(c) on a un isomorphisme $\operatorname{Div}_{\left(\mathbb{A}^{l} \times T\right)_{\bar{k}} \backslash\left(T_{0} \times T\right)_{\bar{k}}}\left(\left(\mathbb{A}^{l} \times T\right)_{\bar{k}}\right) \stackrel{f^{\prime *}}{\longrightarrow} \operatorname{Div}_{X_{\bar{k}} \backslash U_{\bar{k}}}\left(X_{\bar{k}}\right)$.

(2) Soit $G$ un groupe linéaire muni d'un homomorphisme $G \stackrel{\varphi}{\rightarrow} T_{0} \times T$. Supposons que $X$ est une $G$-variété, $U \subset X$ est un $G$-ouvert et $f$ est un $G$-morphisme. Alors le morphisme $f^{\prime}$ est un $G$-morphisme, où l'action de $G$ sur $\mathbb{A}^{l} \times T$ est induite par $\widetilde{\phi} \circ \varphi$.

Démonstration. Soient $\left\{D_{i}\right\}_{i=1}^{l}$ les composantes irréductibles de $(X \backslash U)_{\bar{k}}$ dont la dimension est $\operatorname{dim}(X)-1$. Alors $\operatorname{Div}_{X_{\bar{k}} \backslash U_{\bar{k}}}\left(X_{\bar{k}}\right) \cong \oplus_{i} \mathbb{Z} \cdot\left[D_{i}\right]$. Puisque $\Lambda$ est un isomorphisme, il existe une base $\left\{x_{i}\right\}$ de $T_{0}^{*}$ telle que $\Lambda\left(x_{i}\right)=D_{i}$. Puisque $\bar{k}\left[T_{0}\right]^{\times}=\bar{k}^{\times} \oplus T_{0}^{*}$, on peut supposer que $x_{i} \in \bar{k}\left[T_{0}\right]^{\times}$et $\left\{x_{i}\right\}_{i=1}^{l}$ est globalement $\operatorname{Gal}(\bar{k} / k)$-invariant. Soit $K$ la $k$-algèbre finie séparable qui correspond au $\operatorname{Gal}(\bar{k} / k)$-ensemble $\left\{x_{i}\right\}_{i=1}^{l}$. Alors $T_{0} \cong \operatorname{Res}_{K / k} \mathbb{G}_{m, K}$, et l'immersion ouverte

$$
T_{0, \bar{k}} \cong \operatorname{Spec} \bar{k}\left[x_{1}, x_{1}^{-1}, \cdots, x_{l}, x_{l}^{-1}\right] \hookrightarrow \operatorname{Spec} \bar{k}\left[x_{1}, \cdots, x_{l}\right] \cong \mathbb{A}_{\bar{k}}^{l} \cong\left(\operatorname{Res}_{K / k} \mathbb{A}_{K}^{1}\right)_{\bar{k}}
$$

est exactement l'immersion ouverte $\left(\operatorname{Res}_{K / k} \mathbb{G}_{m, K} \hookrightarrow \operatorname{Res}_{K / k} \mathbb{A}_{K}^{1}\right)_{\bar{k}}$. Puisque $\mathbb{A}^{l} \cong \operatorname{Res}_{K / k} \mathbb{A}_{K}^{1}$, on obtient canoniquement une variété torique $T_{0} \hookrightarrow \mathbb{A}^{l}$.

Notons $T_{\bar{k}} \cong \operatorname{Spec} \bar{k}\left[t_{1}, t_{1}^{-1}, \cdots, t_{n}, t_{n}^{-1}\right]$ et donc

$$
\left(\mathbb{A}^{l} \times T\right)_{\bar{k}} \cong \operatorname{Spec} \bar{k}\left[x_{1}, \cdots, x_{l}, t_{1}, t_{1}^{-1}, \cdots, t_{n}, t_{n}^{-1}\right] .
$$

Soit $T_{0} \stackrel{\phi}{\rightarrow} T$ l'homomorphisme correspondant à la composition

$$
\phi^{*}: \quad T^{*} \stackrel{p_{2}^{*}}{\longrightarrow} T_{0}^{*} \times T^{*} \stackrel{f^{*}}{\longrightarrow} \bar{k}[U]^{\times} / \bar{k}^{\times} \stackrel{\operatorname{div}_{X}}{\longrightarrow} \operatorname{Div}_{X_{\bar{k}} \backslash U_{\bar{k}}}\left(X_{\bar{k}}\right) \stackrel{\Lambda^{-1}}{\longrightarrow} T_{0}^{*} .
$$

Puisque l'homomorphisme $T_{0}^{*} \times T^{*} \stackrel{\widetilde{\phi}^{*}}{\longrightarrow} T_{0}^{*} \times T^{*}$ est défini par $\left(t_{0}, t\right) \mapsto\left(t_{0}-\phi^{*}(t), t\right)$, on a

$$
\left(\operatorname{div}_{X} \circ f^{*} \circ \widetilde{\phi}^{*}\right)\left(x_{i}\right)=\left(\operatorname{div}_{X} \circ f^{*}\right)\left(x_{i}\right)=\Lambda\left(x_{i}\right)=D_{i}
$$

et

$\left(\operatorname{div}_{X} \circ f^{*} \circ \widetilde{\phi}^{*}\right)\left(t_{i}\right)=\left(\left(\operatorname{div}_{X} \circ f^{*}\right)-\left(\operatorname{div}_{X} \circ f^{*} \circ p_{1}^{*} \circ \phi^{*}\right)\right)\left(t_{i}\right)=\left(\left(\operatorname{div}_{X} \circ f^{*}\right)-\left(\Lambda \circ \phi^{*}\right)\right)\left(t_{i}\right)=0$.

Puisque $X$ est lisse, et donc normale, on a

$$
(\widetilde{\phi} \circ f)^{*}\left(\bar{k}\left[\mathbb{A}^{l} \times T\right]\right) \subset \bar{k}[X] \quad \text { et donc }(\tilde{\phi} \circ f)^{*}\left(k\left[\mathbb{A}^{l} \times T\right]\right) \subset k[X] .
$$

Alors $\widetilde{\phi} \circ f$ s'étend en un morphisme $X \stackrel{f^{\prime}}{\rightarrow} \mathbb{A}^{l} \times T$. 
Pour (c), puisque dans le diagramme commutatif

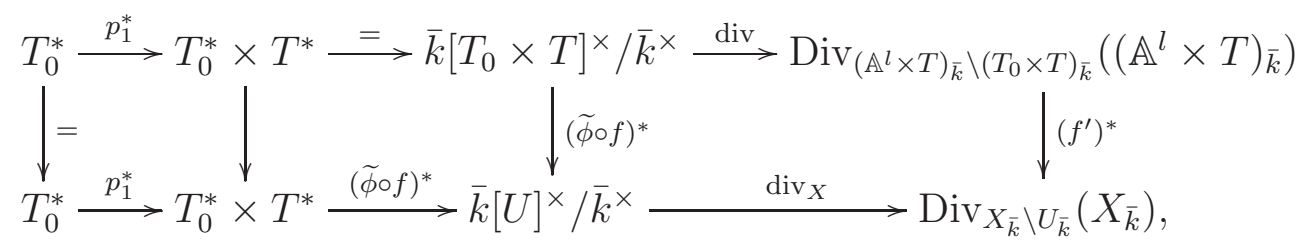

les compositions horizontales sont des isomorphismes, $\left(f^{\prime}\right)^{*}$ est un isomorphisme.

Pour (2), puisque le diagramme

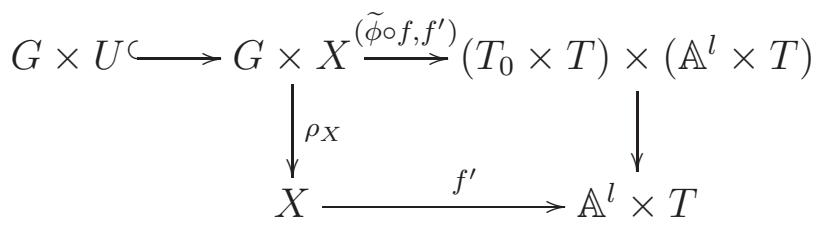

est commutatif en $G \times U$, ce diagramme est commutatif et $f^{\prime}$ est un $G$-morphisme.

2.2. Trivialisation d'un torseur. Les torseurs sous un tore ou sous un groupe de type multiplicatif sont étudiés par Colliot-Thélène et Sansuc [CTSb]. Soit $T$ un tore. Soient $X$ une variété lisse géométriquement intègre et $U \subset X$ un ouvert. Par [CTSb, Lem. 1.6.2], on a une suite exacte canonique :

$$
H^{0}(U, T) \stackrel{\Phi}{\longrightarrow} \operatorname{Hom}_{k}\left(T^{*}, \operatorname{Div}_{X_{\bar{k}} \backslash U_{\bar{k}}}\left(X_{\bar{k}}\right)\right) \stackrel{\Psi}{\longrightarrow} H^{1}(X, T) \longrightarrow H^{1}(U, T),
$$

et pour chaque $\chi \in H^{0}(U, T)=\operatorname{Mor}(U, T)$, on a $\Phi(\chi): T^{*} \stackrel{\chi^{*}}{\longrightarrow} \bar{k}[U]^{\times} / \bar{k}^{\times} \stackrel{\operatorname{div}_{X}}{\longrightarrow} \operatorname{Div}_{X_{\bar{k}} \backslash U_{\bar{k}}}\left(X_{\bar{k}}\right)$.

Pour un $T$-torseur $Y \stackrel{p}{\rightarrow} X$ tel que $\left.Y\right|_{U}$ soit trivial, on note $V:=Y \times_{X} U$ et $\operatorname{Triv}_{U}(V, T)$ l'ensemble des trivialisations $V \stackrel{\tau}{\rightarrow} T \times U$. On a une application canonique

$$
\operatorname{Triv}_{U}(V, T) \stackrel{\Upsilon}{\rightarrow} \operatorname{Hom}_{k}\left(T^{*}, \operatorname{Div}_{X_{\bar{k}} \backslash U_{\bar{k}}}\left(X_{\bar{k}}\right)\right)
$$

définie par : pour chaque $\tau \in \operatorname{Triv}_{U}(V, T)$, le morphisme $\Upsilon(\tau)$ est la composition :

$$
T^{*} \stackrel{p_{1}^{*}}{\longrightarrow} \bar{k}[T \times U]^{\times} / \bar{k}^{\times} \stackrel{\tau^{*}}{\longrightarrow} \bar{k}[V]^{\times} / \bar{k}^{\times} \stackrel{\operatorname{div}_{Y}}{\longrightarrow} \operatorname{Div}_{Y_{\bar{k}} \backslash V_{\bar{k}}}\left(Y_{\bar{k}}\right) \stackrel{\left(p^{*}\right)^{-1}}{\longrightarrow} \operatorname{Div}_{X_{\bar{k}} \backslash U_{\bar{k}}}\left(X_{\bar{k}}\right),
$$

où $\operatorname{Div}_{X_{\bar{k}} \backslash U_{\bar{k}}}\left(X_{\bar{k}}\right) \stackrel{p^{*}}{\rightarrow} \operatorname{Div}_{Y_{\bar{k}} \backslash V_{\bar{k}}}\left(Y_{\bar{k}}\right)$ est un isomorphisme par [CT08, Lem. B.1]. On a aussi une application canonique

$$
H^{0}(U, T) \times \operatorname{Triv}_{U}(V, T) \stackrel{\Theta}{\rightarrow} \operatorname{Triv}_{U}(V, T): \quad(\chi, \tau) \mapsto \widehat{\chi} \circ \tau
$$

où $T \times U \stackrel{\widehat{x}}{\rightarrow} T \times U:(t, u) \mapsto(t+\chi(u), u)$.

Lemme 2.4. L'application $\Theta$ induit une action transitive de $H^{0}(U, T)$ sur $\operatorname{Triv}_{U}(V, T)$, et pour chaque $\tau \in \operatorname{Triv}_{U}(V, T)$, $\chi \in H^{0}(U, T)$, on a $\Upsilon(\Theta(\chi, \tau))=\Phi(\chi)+\Upsilon(\tau)$.

Démonstration. Pour $\chi_{1}, \chi_{2} \in H^{0}(U, T)$, on a $\widehat{\chi_{1}+\chi_{2}}=\widehat{\chi_{2}} \circ \widehat{\chi_{1}}$, et donc $\Theta$ est une action.

Notons $T \times U \stackrel{p_{1}}{\rightarrow} T$. Pour deux trivialisations $\tau_{1}, \tau_{2} \in \operatorname{Triv}_{U}(V, T)$, le morphisme $\tau_{2} \circ \tau_{1}^{-1}$ est un $T$-morphisme sur $U$, i.e. pour chaque $t \in T$ et $u \in U$, on a

$$
\left(\tau_{2} \circ \tau_{1}^{-1}\right)(t, u)=\left(\left(p_{1} \circ \tau_{2} \circ \tau_{1}^{-1}\right)(t, u), u\right)=\left(t+\left(p_{1} \circ \tau_{2} \circ \tau_{1}^{-1}\right)\left(e_{T}, u\right), u\right) .
$$


Notons $\chi:=\left(p_{1} \circ \tau_{2} \circ \tau_{1}^{-1}\right)\left(e_{T},-\right) \in H^{0}(U, T)$, alors $\tau_{2}=\widehat{\chi} \circ \tau_{1}$ et donc $\Theta$ est transitive.

Notons $T \times U \stackrel{p_{2}}{\rightarrow} U$. Pour tout $\tau \in \operatorname{Triv}_{U}(V, T)$ et tout $\chi \in H^{0}(U, T)$, on a

$$
p_{1} \circ \Theta(\chi, \tau)=p_{1} \circ \widehat{\chi} \circ \tau=\left(p_{1}+\chi \circ p_{2}\right) \circ \tau=p_{1} \circ \tau+\chi \circ p_{2} \circ \tau=p_{1} \circ \tau+\left.\chi \circ p\right|_{U}
$$

dans $\operatorname{Mor}(V, T)$. Puisque les deux morphismes composés

$\bar{k}[U]^{\times} / \bar{k}^{\times} \stackrel{\left.p\right|_{U} ^{*}}{\longrightarrow} \bar{k}[V]^{\times} / \bar{k}^{\times} \stackrel{\operatorname{div}_{Y}}{\longrightarrow} \operatorname{Div}_{Y_{\bar{k}} \backslash V_{\bar{k}}}\left(Y_{\bar{k}}\right)$ et $\bar{k}[U]^{\times} / \bar{k}^{\times} \stackrel{\operatorname{div}_{X}}{\longrightarrow} \operatorname{Div}_{X_{\bar{k}} \backslash U_{\bar{k}}}\left(X_{\bar{k}}\right) \stackrel{p^{*}}{\longrightarrow} \operatorname{Div}_{Y_{\bar{k}} \backslash V_{\bar{k}}}\left(Y_{\bar{k}}\right)$

coïncident, on a $\operatorname{div}_{Y} \circ\left(\left.\chi \circ p\right|_{U}\right)^{*}=p^{*} \circ \operatorname{div}_{X} \circ \chi^{*}$ et le résultat en découle.

Proposition 2.5. Avec les notations des (2.2) et (2.3), pour un $\phi \in \operatorname{Hom}_{k}\left(T^{*}, \operatorname{Div}_{X_{\bar{k}} \backslash U_{\bar{k}}}\left(X_{\bar{k}}\right)\right)$, soit $Y \rightarrow X$ un torseur correspondant à $\Psi(\phi)$ et soit $V:=Y \times_{X} U$. Alors après avoir bien choisi le signe de $\Psi$, il existe une trivialisation $\tau \in \operatorname{Triv}_{U}(V, T)$ telle que $\Upsilon(\tau)=\phi$ et que, pour chaque $\tau^{\prime} \in \operatorname{Triv}_{U}(V, T)$, on ait $\Psi\left(\Upsilon\left(\tau^{\prime}\right)\right)=[Y]$.

Démonstration. D'après le lemme 2.4, l'énoncé est équivalent à l'existence d'une trivialisation $\tau \in \operatorname{Triv}_{U}(V, T)$ telle que $\Psi(\Upsilon(\tau))=[Y]$.

Étape (1). Supposons que $k=\bar{k}$ et $T=\mathbb{G}_{m}$. Dans ce cas, $T^{*} \cong \mathbb{Z}$. La suite exacte (2.2) est obtenue en appliquant $H^{i}(X,-)=\operatorname{Ext}_{X_{\text {ét }}}^{i}(\mathbb{Z},-)$ à la suite exacte de faisceaux (cf. [CTSb, Lem. 1.6.2])

$$
0 \rightarrow \mathbb{G}_{m, X} \rightarrow j_{*} \mathbb{G}_{m, U} \rightarrow \operatorname{Div}_{X \backslash U}(X) \rightarrow 0
$$

où $U \stackrel{j}{\rightarrow} X$. Donc, après avoir choisi $1_{T^{*}} \in T^{*}$, le morphisme

$$
\operatorname{Div}_{X \backslash U}(X) \stackrel{\sim}{\leftarrow} \operatorname{Hom}_{k}\left(T^{*}, \operatorname{Div}_{X \backslash U}(X)\right) \stackrel{\Psi}{\rightarrow} H^{1}(X, T) \stackrel{\sim}{\rightarrow} \operatorname{Pic}(X)
$$

est le morphisme canonique $\operatorname{Div}_{X \backslash U}(X) \rightarrow \operatorname{Pic}(X)$. Par [CT08, Lem. B.1], pour chaque trivialisation $\tau$, on a un diagramme commutatif :

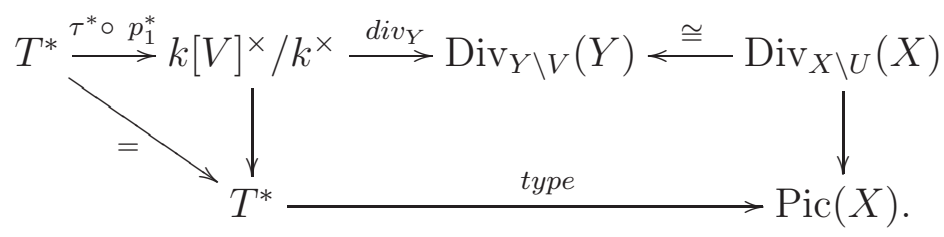

Puisque type $\left(1_{T^{*}}\right)=[Y]$, le résultat en découle.

Étape (2). Supposons $k=\bar{k}$. Notons $n=\operatorname{dim}(T)$. Alors $T \cong \mathbb{G}_{m}^{n}, T^{*} \cong \mathbb{Z}^{n}, H^{1}(X, T) \cong$ $\operatorname{Pic}(X)^{\oplus n}$ et $\operatorname{Hom}\left(T^{*}, \operatorname{Div}_{X \backslash U}(X)\right) \cong \operatorname{Div}_{X \backslash U}(X)^{\oplus n}$. On se réduit ainsi à l'étape (1).

Étape (3). Supposons que $T$ est quasi-trivial et $X$ est projective. Dans ce cas, le morphisme $H^{1}(X, T) \rightarrow H^{1}\left(X_{\bar{k}}, T\right)$ est injectif (une conséquence de [CTSb, (2.0.2)]). Par l'étape (2), pour chaque $\tau \in \operatorname{Triv}_{U}(V, T)$, on a $\left.\Psi(\Upsilon(\tau))\right|_{\bar{k}}=\left[Y_{\bar{k}}\right]$, et donc $\Psi(\Upsilon(\tau))=[Y]$.

Étape (4). En général, soit $X^{c}$ une compactification lisse de $X$ et soit $T_{0}$ un tore avec un isomorphisme $T_{0}^{*} \stackrel{\iota}{\rightarrow} \operatorname{Div}_{X_{\bar{k}} \backslash U_{\bar{k}}}\left(X_{\bar{k}}\right)$. Notons $\sigma^{*}:=\iota^{-1} \circ \phi$ et $T_{0} \stackrel{\sigma}{\rightarrow} T$ le morphisme correspondant de tores. L'égalité $\operatorname{Div}_{X_{\bar{k}}^{c} \backslash U_{\bar{k}}}\left(X_{\bar{k}}^{c}\right) \cong \operatorname{Div}_{X_{\bar{k}}^{c} \backslash X_{\bar{k}}}\left(X_{\bar{k}}^{c}\right) \oplus \operatorname{Div}_{X_{\bar{k}} \backslash U_{\bar{k}}}\left(X_{\bar{k}}\right)$ donne des morphismes

$$
\iota_{c}: T_{0}^{*} \stackrel{\iota}{\rightarrow} \operatorname{Div}_{X_{\bar{k}} \backslash U_{\bar{k}}}\left(X_{\bar{k}}\right) \hookrightarrow \operatorname{Div}_{X_{\bar{k}}^{c} \backslash U_{\bar{k}}}\left(X_{\bar{k}}^{c}\right) \quad \text { et } \pi: \operatorname{Div}_{X_{\bar{k}}^{c} \backslash U_{\bar{k}}}\left(X_{\bar{k}}^{c}\right) \rightarrow \operatorname{Div}_{X_{\bar{k}} \backslash U_{\bar{k}}}\left(X_{\bar{k}}\right)
$$


tels que $\pi \circ \iota_{c}=\iota$. Par la suite exacte (2.2), on a un diagramme commutatif

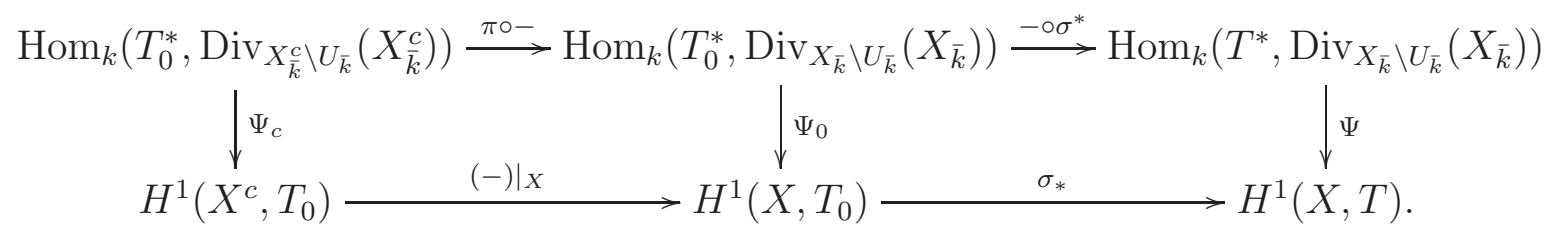

Alors $\Psi_{c}\left(\iota_{c}\right)$ donne un $T_{0}$-torseur $Y_{0}^{c}$ sur $X^{c}$ tel que $\pi \circ \iota_{c} \circ \sigma^{*}=\phi, \sigma_{*}\left[\left.Y_{0}^{c}\right|_{X}\right]=[Y] \in H^{1}(X, T)$ et que $V_{0}:=\left.Y_{0}^{c}\right|_{U} \cong T_{0} \times U$. Ceci donne un diagramme commutatif :

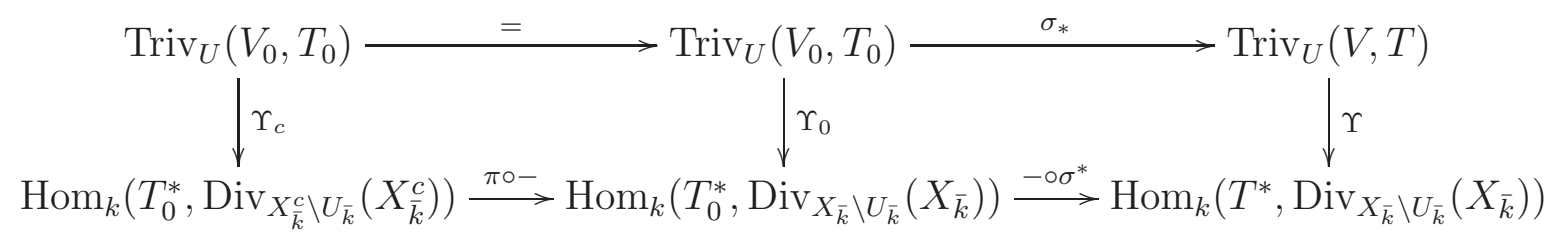

et le résultat découle de l'étape (3).

2.3. L'action d'un groupe sur un torseur. Soit $S$ un groupe de type multiplicatif et soit $G$ un groupe linéaire connexe. Colliot-Thélène a montré que tout $S$-torseur $H$ sur $G$ peut être muni d'une structure de groupe telle que $H \rightarrow G$ soit un homomorphisme de groupes, s'il possède un point rationnel au-dessus de $e_{G}$ (cf. [CT08, Thm. 5.6]). On donne une généralisation de ce résultat (Théorème 2.7).

On commence par une généralisation de [CT08, Lem. 5.5].

Lemme 2.6. Soient $X_{1}, X_{2}$ deux variétés lisses géométriquement intègres. Soit $S$ un groupe de type multiplicatif. Supposons que $X_{1}$ est géométriquement rationnelle et $X_{1}(k) \neq \emptyset$. Alors pour chaque $e \in X_{1}(k)$ et $i=0$ ou 1 , on a un isomorphisme canonique :

$$
H_{e}^{i}\left(X_{1}, S\right) \oplus H^{i}\left(X_{2}, S\right) \stackrel{\sim}{\rightarrow} H^{i}\left(X_{1} \times X_{2}, S\right)
$$

ò̀ $H_{e}^{i}\left(X_{1}, S\right):=\operatorname{Ker}\left(H^{i}\left(X_{1}, S\right) \stackrel{e^{*}}{\rightarrow} H^{i}(k, S)\right)$.

Démonstration. Si $S=\mathbb{G}_{m}$, l'énoncé découle de [S, Lem. 6.5 et Lem. 6.6]. Si $S$ est quasitrivial, i.e. il existe une $k$-algèbre finie étale $K$ tel que $S=\operatorname{Res}_{K / k} \mathbb{G}_{m}$, l'énoncé découle du fait $H^{1}(-, S) \cong H^{1}\left((-)_{K}, \mathbb{G}_{m}\right)$.

En général, on note $H_{1}^{i}(S):=H_{e}^{i}\left(X_{1}, S\right) \oplus H^{i}\left(X_{2}, S\right)$ et $H_{2}^{i}(S):=H^{i}\left(X_{1} \times X_{2}, S\right)$ pour $i=0$ ou 1. Il existe un tore $T$, un tore quasi-trivial $T_{0}$ et une suite exacte :

$$
0 \rightarrow S \rightarrow T_{0} \rightarrow T \rightarrow 0 .
$$

Elle induit un diagramme commutatif de suites exactes :

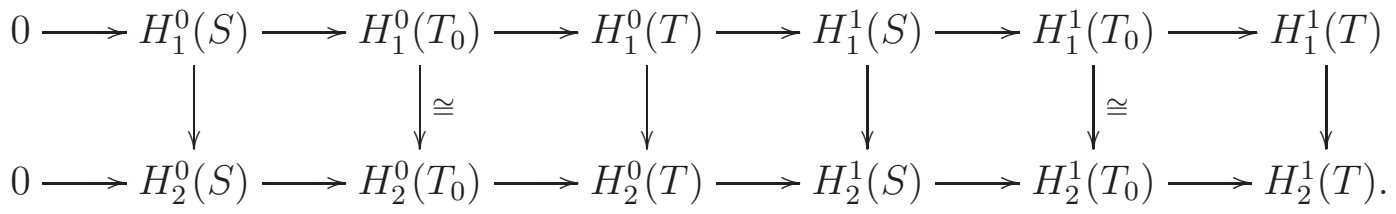

Le résultat découle du lemme des cinq. 
Théorème 2.7. Soient $G$ un k-groupe linéaire connexe et $(X, \rho)$ une G-variété lisse géométriquement intègre. Soient $S$ un groupe de type multiplicatif et $Y \stackrel{p}{\rightarrow} X$ un $S$-torseur. Alors il existe un groupe linéaire $H$ et un homomorphisme $H \stackrel{\psi}{\rightarrow} G$ tels que $\psi$ soit surjectif de noyau $S$ central et que l'action $\rho_{S}$ de $S$ en $Y$ s'étende une action $\rho_{H}$ de $H$ sur $Y$ qui fait $p$ un $H$-morphisme.

De plus :

(1) le groupe $H$ est unique, i.e. si $H_{1} \stackrel{\psi_{1}}{\longrightarrow} G$ satisfait les conditions ci-dessus, alors il existe un isomomorphisme de $k$-groupes $H_{1} \stackrel{\vartheta}{\rightarrow} H$ tel que l'on ait un diagramme commutatif :

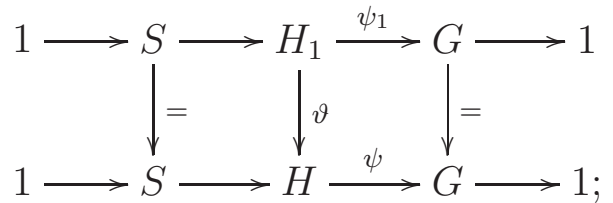

(2) si $\rho^{*}[Y]=p_{2}^{*}[Y]$ dans $H^{1}(G \times X, S)$, alors $H \cong S \times G$;

(3) pour chaque choix d'une action $\rho_{H}$, chaque homomorphisme $G \stackrel{\phi}{\rightarrow} S$ induit une nouvelle action $H \times Y \stackrel{\rho_{H, \phi}}{\longrightarrow} Y$ satisfaisant les conditions ci-dessus, où

$$
\rho_{H, \phi}(h, y)=(\phi \circ \psi)(h) \cdot \rho_{H}(h, y) \quad \text { pour chaque } h \in H, y \in Y,
$$

et toutes les actions satisfaisant les conditions ci-dessus sont obtenues de cette façon.

Démonstration. On suit la démonstration de Colliot-Thélène [CT08, Thm. 5.6]. D'après le lemme 2.6, pour chaque élément $\alpha \in H^{1}(X, S)$, puisque $\left.\rho^{*}(\alpha)\right|_{e_{G} \times X}=\alpha$, il existe un unique $\beta \in H_{e_{G}}^{1}(G, S)$ tel que $\rho^{*}(\alpha)=p_{2}^{*}(\alpha)+p_{1}^{*}(\beta)$, où $p_{1}, p_{2}$ sont les projections. Si $\alpha=[Y]$, on note $\beta=(H \stackrel{\psi}{\rightarrow} G)$. Par [CT08, Thm. 5.6 et Cor. 5.7], il existe une structure unique de $k$-groupe linéaire sur $H$ (à (2.5) près) telle que $\psi$ soit un homomorphisme de noyau $S$ central.

Notons $S \stackrel{i}{\rightarrow} H$ l'immersion. L'égalité $\rho^{*}[Y]=p_{1}^{*}[H]+p_{2}^{*}[Y]$ donne un diagramme commutatif :

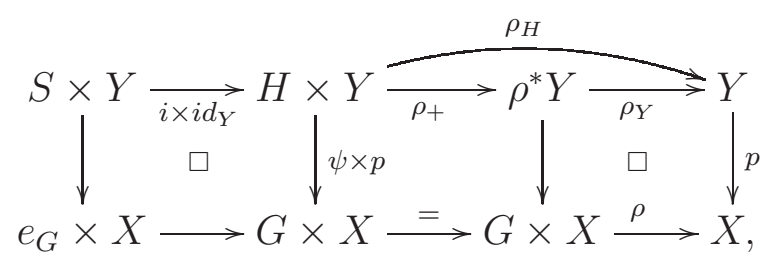

tel que $\rho_{+}$induise un isomorphisme $H \times{ }^{S} Y \stackrel{\sim}{\rightarrow} \rho^{*} Y$ et $\rho_{H}:=\rho_{Y} \circ \rho_{+}$soit un $S \times S$-morphisme, où l'action $S \times S \curvearrowright Y:\left(s_{1}, s_{2}, y\right) \mapsto \rho_{S}\left(s_{1} \cdot s_{2}, y\right)$. Donc $\left.\rho_{H}\right|_{e_{S} \times Y} \in \operatorname{Hom}_{X}(Y, Y)$ est un $S$ morphisme, et donc un isomorphisme. Remplaçant $\rho_{Y}$ par $\left(\left.\rho_{H}\right|_{e_{S} \times Y}\right)^{-1} \circ \rho_{Y}$, on peut supposer que $\left.\rho_{H}\right|_{e_{S} \times Y}$ est l'identité, et donc $\rho_{S}=\rho_{H} \circ\left(i \times i d_{Y}\right)$.

Montrons que $\rho_{H}$ est une action. Notons $m_{H}$ la multiplication sur $H$. Puisque $\rho_{G}$ est une action, les morphismes $\rho_{1}:=\rho_{H} \circ\left(m_{H} \times i d_{Y}\right)$ et $\rho_{2}:=\rho_{H} \circ\left(i d_{H} \times \rho_{H}\right)$ induisent un morphisme $\Psi: H \times H \times Y \stackrel{\left(\rho_{1}, \rho_{2}\right)}{\longrightarrow} Y \times_{X} Y \stackrel{\sim}{\rightarrow} Y \times S \stackrel{p_{2}}{\longrightarrow} S, \quad$ i.e. $\rho_{H}\left(h_{1} \cdot h_{2}, y\right)=\Psi\left(h_{1}, h_{2}, y\right) \cdot \rho_{H}\left(h_{1}, \rho_{H}\left(h_{2}, y\right)\right)$ pour chaque $h_{1}, h_{2} \in H$ et $y \in Y$. Puisque $\rho_{H}$ est un $S \times S$-morphisme, il existe un morphisme $G \times G \times X \stackrel{\Psi_{1}}{\longrightarrow} S$ tel que $\Psi=\Psi_{1} \circ(\psi \times \psi \times p)$. Par le lemme de Rosenlicht (voir [S, Lem. 6.5]), 
il existe des homomorphismes $\chi_{1}, \chi_{2}: G \rightarrow S$ et un morphisme $X \stackrel{\chi_{0}}{\rightarrow} S$ tels que

$$
\Psi_{1}\left(g_{1}, g_{2}, x\right)=\chi_{1}\left(g_{1}\right) \cdot \chi_{2}\left(g_{2}\right) \cdot \chi_{0}(x)
$$

pour chaque $g_{1}, g_{2} \in G$ et $x \in X$. Puisque $\rho_{H}\left(e_{H}, y\right)=y$ pour chaque $y \in Y$, on a $\chi_{1}=0$, $\chi_{2}=0$ et $\chi_{0}(x)=e_{S}$ pour chaque $x \in X$. Donc $\rho_{H}$ est une action.

Pour (1), s'il existe un groupe linéaire $H$ qui satisfait l'énoncé du théorème, alors $H$ et l'action $\rho_{H}$ en $Y$ induisent le diagramme (2.6) tel que $\rho_{H}:=\rho_{Y} \circ \rho_{+}$et $H \times^{S} Y \stackrel{\sim}{\rightarrow} \rho^{*} Y$. Alors dans $H^{1}(G \times X, S)$, on a $p_{1}^{*}[H]+p_{2}^{*}[Y]=\rho^{*}[Y]$, ce qui détermine uniquement $H$ par l'argument ci-dessus.

Pour (2), si $\rho^{*}[Y]=p_{2}^{*}[Y]$, on a $[H]=0$ et, d'après (1), on a $H \cong S \times G$.

Pour (3), il est clair que $\left.\rho_{H, \phi}\right|_{S \times Y}=\rho_{S}$ et $p \circ \rho_{H, \phi}=\rho \circ(\psi \times p)$. Par ailleurs, soit $\rho_{H}^{\prime}$ une action satisfaisant les conditions. Alors on a un morphisme

$$
\Phi: H \times Y \stackrel{\left(\rho_{H}, \rho_{H}^{\prime}\right)}{\longrightarrow} Y \times_{X} Y \stackrel{\sim}{\rightarrow} Y \times S \stackrel{p_{2}}{\longrightarrow} S \quad \text { i.e. } \quad \rho_{H}^{\prime}(h, y)=\Phi(y, h) \cdot \rho_{H}(h, y)
$$

pour chaque $h \in H$ et $y \in Y$. Puisque $\left.\rho_{H}^{\prime}\right|_{S \times Y}=\rho_{S}$, il existe $G \times X \stackrel{\Phi_{1}}{\rightarrow} S$ tel que $\Phi=\Phi_{1} \circ(\psi \times p)$. Par le lemme de Rosenlicht (voir [S, Lem. 6.5]), il existe un homomorphisme $G \stackrel{\phi}{\rightarrow} S$ et un morphisme $X \stackrel{\chi}{\rightarrow} S$ tels que $\Phi_{1}(g, x)=\phi(g) \cdot \chi(x)$ pour chaque $g \in G$ et $x \in X$. Puisque $\rho_{H}^{\prime}\left(e_{H}, y\right)=y$ pour chaque $y \in Y$, on a $\chi(x)=e_{S}$ pour tout $x \in X$. Donc $\rho_{H}^{\prime}=\rho_{H, \phi}$.

Corollaire 2.8. Soient $G$ un k-groupe linéaire connexe et $X_{1}, X_{2}$ deux $G$-variétés lisses géométriquement intègres munies d'un $G$-morphisme $X_{1} \rightarrow X_{2}$. Soit $S$ un groupe de type multiplicatif. Pour $i=1,2$, soient $Y_{i} \rightarrow X_{i}$ un $S$-torseur et $H_{i}$ le groupe linéaire donné par le théorème 2.7. Si $Y_{1} \cong Y_{2} \times_{X_{2}} X_{1}$ comme $S$-torseurs, alors $H_{1} \cong H_{2}$ et, après avoir changé l'action de $H_{1}$ sur $Y_{1}$ ou l'action de $H_{2}$ sur $Y_{2}$, on peut imposer que $Y_{1} \cong Y_{2} \times_{X_{2}} X_{1} \rightarrow Y_{2}$ soit un $H_{1}$-morphisme.

Démonstration. L'action de $H_{2}$ sur $Y_{2}$ induit canoniquement une action de $H_{2}$ sur $Y_{2} \times_{X_{2}} X_{1}$ telle que $Y_{2} \times_{X_{2}} X_{1} \rightarrow X_{1}$ soit un $H_{2}$-morphisme. Par l'unicité dans le théorème $2.7, H_{1} \cong H_{2}$. Le résultat découle du fait que la différence de deux actions est un homomorphisme $G \rightarrow S$, qui ne dépend pas de $X_{i}$.

Corollaire 2.9. Sous les hypothèses du théorème 2.7, si le S-torseur $[Y]$ est trivial sur un $G$-ouvert $U$ de $X$, alors $H \cong S \times G$.

Démonstration. Le résultat découle du théorème 2.7 (1) et (2).

Corollaire 2.10. Sous les hypothèses du théorème 2.7, supposons que $X \cong G / G_{0}$, où $G_{0} \subset G$ est un sous-groupe fermé connexe. Si $Y(k) \neq \emptyset$, alors il existe un sous-groupe connexe fermé $H_{0}$ de $H$ tel que $Y \cong H / H_{0}$ et $H_{0} \cong G_{0}$.

Démonstration. Soient $y \in Y(k), x:=p(y)$ et $G \stackrel{\pi}{\rightarrow} X$ le morphisme induit par $x$. Alors $Y_{G}:=Y \times_{X} G$ a un $k$-point sur $e_{G}$. Par [CT08, Thm. 5.6], $Y_{G}$ est un groupe satisfaisant les conditions du théorème 2.7. Par le corollaire 2.8, $Y_{G} \cong H$ et $H \stackrel{\pi_{Y}}{\longrightarrow} Y$ est un $H$-morphisme. Donc $H_{0}:=\pi_{Y}^{-1}(y) \cong \pi^{-1}(x) \cong G_{0}$.

\section{Groupe De Brauer invariant}

Dans toute cette section, $k$ est un corps quelconque de caractéristique 0. Sauf mention explicite, une variété est une $k$-variété. Soit $G$ un groupe algébrique et soit $X$ une $G$-variété lisse. 
On définit la notion de sous-groupe $G$-invariant de $\operatorname{Br}(X)$ (Définition 3.1). Ensuite on établit "l'algébricité" de $\operatorname{Br}_{G}(X)$ (Proposition 3.7). On définit la notion d'homomorphisme de Sansuc (cf. Définition 3.8) et on obtient un diagramme commutatif canonique de suites exactes de Sansuc (Théorème 3.10).

\subsection{Définitions et propriétés.}

Définition 3.1. Soient $G$ un groupe algébrique connexe et $(X, \rho)$ une $G$-variété lisse géométriquement intègre. Le sous-groupe de Brauer $G$-invariant de $X$ est le sous-groupe

$$
\operatorname{Br}_{G}(X):=\left\{b \in \operatorname{Br}(X):\left(\rho^{*}(b)-p_{2}^{*}(b)\right) \in p_{1}^{*} \operatorname{Br}(G)\right\}
$$

de $\operatorname{Br}(X)$, où $G \times X \stackrel{p_{1}}{\rightarrow} G, G \times X \stackrel{p_{2}}{\rightarrow} X$ sont les projections.

Dans la proposition 3.3, pour un sous-groupe $B \subset \operatorname{Br}(X)$, on montre que $B \subset \operatorname{Br}_{G}(X)$ si et seulement si $B$ est " $G$-invariant".

Le lemme suivant est bien connu.

Lemme 3.2. Soient $X, Y$ deux variétés lisses géométriquement intègres et $Y \stackrel{p}{\rightarrow} X$ un morphisme fidèlement plat à fibres géométriquement intègres. Soient $U$ un ouvert non vide de $X$ et $V:=p^{-1}(U)$. Soit $B \subset \operatorname{Br}(U)$ un sous-groupe. Alors

$$
p^{*}(B \cap \operatorname{Br}(X))=\left(p^{*} B\right) \cap \operatorname{Br}(Y) .
$$

Proposition 3.3. Sous les hypothèses de la Définition 3.1, pour un sous-groupe $B \subset \operatorname{Br}(X)$, les énoncés ci-dessous sont équivalents :

(1) $B \subset \mathrm{Br}_{G}(X)$;

(2) $\rho^{*} B+p_{1}^{*} \operatorname{Br}(G)=p_{2}^{*} B+p_{1}^{*} \operatorname{Br}(G)$;

(3) pour toute extension de corps $K / k$ et tout $g \in G(K)$, l'action $\rho_{g}: X_{K} \stackrel{g \cdot(-)}{\longrightarrow} X_{K}$ induit un morphisme $\operatorname{Br}\left(X_{K}\right) \stackrel{\rho_{g}^{*}}{\rightarrow} \operatorname{Br}\left(X_{K}\right)$ tel que

$$
\rho_{g}^{*} \pi^{*} B+\operatorname{ImBr}(K)=\pi^{*} B+\operatorname{ImBr}(K)
$$

où $X_{K} \stackrel{\pi}{\rightarrow} X$;

(4) pour tout $b \in B$, toute extension de corps $K / k$ et tout $g \in G(K)$, on a

$$
\left(\rho_{g}^{*} \pi^{*}(b)-\pi^{*}(b)\right) \in \operatorname{ImBr}(K),
$$

où $\pi$ et $\rho_{g}^{*}$ sont définis dans (3);

(5) pour toute extension de corps $K / k$ telle que $k$ soit algébriquement clos dans $K$, et tout $g \in G(K)$, on a (3.2), où $\pi$ et $\rho_{g}^{*}$ sont définis dans (3).

Démonstration. Les implications $(4) \Rightarrow(3),(3) \Rightarrow(5)$ sont claires.

Pour $(2) \Rightarrow(1)$, on note $i_{e}: X \stackrel{e_{G} \times i d_{X}}{\longrightarrow} G \times X$ l'immersion fermée. Pour $b \in B$, il existe $b^{\prime} \in B$ et $a \in \operatorname{Br}(G)$ tels que $\rho^{*}(b)=p_{2}^{*}\left(b^{\prime}\right)+p_{1}^{*}(a)$. Puisque $\rho \circ i_{e}=p_{2} \circ i_{e}=i d_{X}$ et que $p_{1} \circ i_{e}$ se factorise par Spec $k$, on a $b-b^{\prime} \in \operatorname{ImBr}(k)$ et donc

$$
\left(\rho^{*}(b)-p_{2}^{*}(b)\right) \in\left(p_{1}^{*} \operatorname{Br}(G)+\operatorname{ImBr}(k)\right)=p_{1}^{*} \operatorname{Br}(G) .
$$

Pour $(1) \Rightarrow(4)$, on note $i_{g}: X \stackrel{g \times i d_{X}}{\longrightarrow} G \times X$ le morphisme et $A:=\operatorname{ImBr}(K)$. Alors $\pi \circ \rho_{g}=\rho \circ i_{g}$ et $\pi=p_{2} \circ i_{g}$. Puisque $i_{g}^{*}\left(p_{1}^{*} \operatorname{Br}(G)\right) \subset A$, on a

$$
\rho_{g}^{*} \pi^{*}(b)+A=i_{g}^{*}\left(p_{1}^{*} \operatorname{Br}(G)+\rho^{*}(b)\right)+A=i_{g}^{*}\left(p_{1}^{*} \operatorname{Br}(G)+p_{2}^{*}(b)\right)+A=\pi^{*}(b)+A .
$$


Pour $(5) \Rightarrow(2)$, notons $X_{\eta_{G}} \stackrel{\pi}{\rightarrow} X$ la projection et $X_{\eta_{G}} \stackrel{i_{\eta}}{\rightarrow} G \times X$ l'immersion canonique. Alors $\operatorname{Br}(G \times X) \rightarrow \operatorname{Br}\left(X_{\eta_{G}}\right)$ est injectif. Par le lemme 3.2, $p_{1}^{*} \operatorname{Br}\left(\eta_{G}\right) \cap \operatorname{Br}(G \times X)=p_{1}^{*} \operatorname{Br}(G)$. Donc il suffit de montrer que :

$$
\left(i_{\eta}^{*} \rho^{*}\right) B+\operatorname{ImBr}\left(\eta_{G}\right)=\left(i_{\eta}^{*} p_{2}^{*}\right) B+\operatorname{ImBr}\left(\eta_{G}\right) .
$$

Le résultat découle de $p_{2} \circ i_{\eta}=\pi$ et $\rho \circ i_{\eta}=\rho_{\eta_{G}} \circ \pi$.

Proposition 3.4. Sous les hypothèses de la Définition 3.1, alors :

(1) pour tout groupe algébrique connexe $G_{0}$ muni d'un homomorphisme $G_{0} \stackrel{\phi}{\rightarrow} G$, on a $\operatorname{Br}_{G}(X) \subset \operatorname{Br}_{G_{0}}(X)$;

(2) pour toute $G$-variété $Y$ lisse géométriquement intègre munie d'un $G$-morphisme $Y \stackrel{p}{\rightarrow} X$, on a $p^{*} \operatorname{Br}_{G}(X) \subset \operatorname{Br}_{G}(Y)$, où $\operatorname{Br}(X) \stackrel{p^{*}}{\rightarrow} \operatorname{Br}(Y)$;

(3) pour tout $G$-ouvert dense $U \subset X$, on a $\operatorname{Br}_{G}(X)=\operatorname{Br}_{G}(U) \cap \operatorname{Br}(X)$;

(4) si $G$ est linéaire, on a $\operatorname{Br}_{1}(X) \subset \operatorname{Br}_{G}(X)$;

(5) pour toute $G$-variété $Y$ munie d'un $G$-morphisme $Y \stackrel{p}{\rightarrow} X$, si $p$ est un torseur sous un groupe linéaire connexe $H$, on a $\left(p^{*}\right)^{-1} \operatorname{Br}_{G}(Y) \subset \operatorname{Br}_{G}(X)$, où $\operatorname{Br}(X) \stackrel{p^{*}}{\rightarrow} \operatorname{Br}(Y)$;

(6) sous les hypothèses de (5), si $G$ est linéaire, on a

$$
\operatorname{Br}_{1}(X, Y):=\operatorname{Ker}\left(\operatorname{Br}(X) \rightarrow \operatorname{Br}\left(Y_{\bar{k}}\right)\right) \subset \operatorname{Br}_{G}(X)
$$

Démonstration. Les énoncés (1), (2) et (3) découlent de la définition.

Pour (4), par [S, Lem. 6.6], $\operatorname{Br}_{1}(G \times X)=\operatorname{Br}_{a}(G) \oplus \operatorname{Br}_{1}(X)$, et donc

$$
\operatorname{Br}_{1}(G \times X)=p_{2}^{*} \operatorname{Br}_{1}(X)+p_{1}^{*} \operatorname{Br}_{1}(G)=\rho^{*} \operatorname{Br}_{1}(X)+p_{1}^{*} \operatorname{Br}_{1}(G) .
$$

Le résultat découle de la proposition 3.3.

Pour (5), puisque $G \times Y \stackrel{i d_{G} \times p}{\longrightarrow} G \times X$ est aussi un $H$-torseur, par la suite exacte de Sansuc ([S, Prop. 6.10]), on a un diagramme commutatif de suites exactes

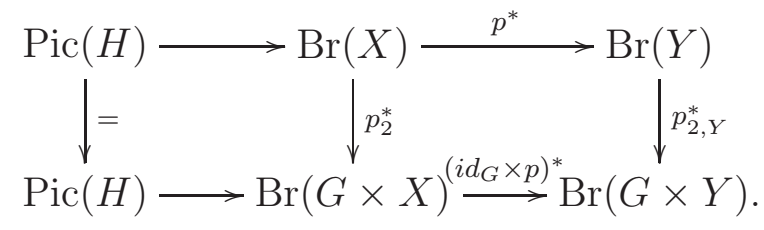

Donc, pour tout $\alpha \in\left(p^{*}\right)^{-1} \operatorname{Br}_{G}(Y)$, on a

$$
\rho^{*}(\alpha)-p_{2}^{*}(\alpha) \in p_{1}^{*} \operatorname{Br}(G)+\operatorname{ImPic}(H) \subset p_{1}^{*} \operatorname{Br}(G)+p_{2}^{*} \operatorname{Br}(X) .
$$

D'après calculer sur $X \stackrel{e_{G} \times i d_{X}}{\longrightarrow} G \times X$, on peut voir que $\rho^{*}(\alpha)-p_{2}^{*}(\alpha) \in p_{1}^{*} \operatorname{Br}(G)$.

Par (4) et (5), $\operatorname{Br}_{1}(X, Y) \subset \operatorname{Br}_{G}(X)$.

Soient $X$ une variété lisse géométriquement intègre et $U \subset X$ un ouvert non vide. Supposons que $D:=(X \backslash U)$ est lisse de codimension 1. Par le théorème de pureté pour la cohomologie étale à support dans un fermé lisse (cf. [Mi80, §VI.5]), on a une suite exacte :

$$
H^{2}(X, \mathbb{Q} / \mathbb{Z}(1)) \rightarrow H^{2}(U, \mathbb{Q} / \mathbb{Z}(1)) \rightarrow H^{1}(D, \mathbb{Q} / \mathbb{Z}) \rightarrow H^{3}(X, \mathbb{Q} / \mathbb{Z}(1)) \rightarrow H^{3}(U, \mathbb{Q} / \mathbb{Z}(1)) .
$$


Puisque $\operatorname{Pic}(X) \rightarrow \operatorname{Pic}(U)$ est surjectif et $\operatorname{Br}(X) \rightarrow \operatorname{Br}(U)$ est injectif, d'après la suite exacte de Kummer, on a la suite exacte (cf. Grothendieck [Gro])

$$
0 \rightarrow \operatorname{Br}(X) \rightarrow \operatorname{Br}(U) \stackrel{\partial}{\rightarrow} H^{1}(D, \mathbb{Q} / \mathbb{Z}) \rightarrow H^{3}(X, \mathbb{Q} / \mathbb{Z}(1)) \rightarrow H^{3}(U, \mathbb{Q} / \mathbb{Z}(1)) .
$$

Soit $G$ un groupe algébrique connexe. Si $X$ est munie d'une $G$-action $\rho: G \times X \rightarrow X$ respectant $U$, on a un diagramme de suites exactes :

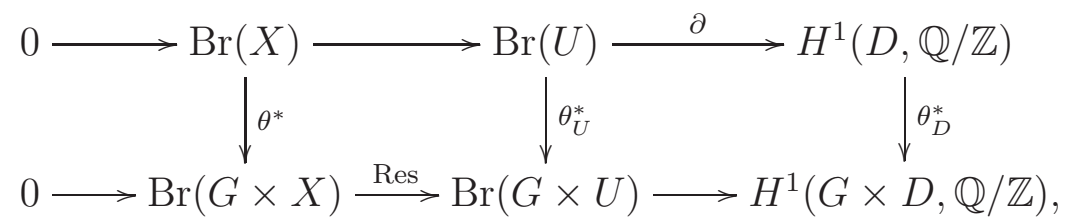

où $G \times X \stackrel{\theta}{\rightarrow} X$ est soit $p_{2}$ soit $\rho$. On a :

Lemme 3.5. Avec les notations et hypothèses ci-dessus, on a :

(1) $\left.p_{2, D}^{*}\right|_{\partial\left(\operatorname{Br}_{G}(U)\right)}=\left.\rho_{D}^{*}\right|_{\partial\left(\operatorname{Br}_{G}(U)\right)}$;

(2) pour tout $b \in \operatorname{Br}_{G}(U)$, il existe un revêtement fini étale galoisien abélien $D^{\prime} \stackrel{\pi}{\rightarrow} D$ tel que $D^{\prime}$ soit une $G$-variété, $\pi$ soit un $G$-morphisme et que $\pi^{*}(\partial(b))=0 \in H^{1}\left(D^{\prime}, \mathbb{Q} / \mathbb{Z}\right)$.

Démonstration. Puisque $p_{1, U}^{*} \operatorname{Br}(G) \subset \operatorname{Im}($ Res), l'énoncé (1) découle de la proposition $3.3(2)$. Pour $b \in \operatorname{Br}_{G}(U)$, soit $n \in \mathbb{Z}$ l'ordre de $\partial(b)$. Alors $\partial(b) \in H^{1}(D, \mathbb{Z} / n)$. Soit $D^{\prime} \stackrel{\pi}{\rightarrow} D$ un $\mathbb{Z} / n$ torseur tel que $\left[D^{\prime}\right]=\partial(b)$. Par $(1), p_{2, D}^{*}\left[D^{\prime}\right]=\rho_{D}^{*}\left[D^{\prime}\right] \in H^{1}(G \times D, \mathbb{Z} / n)$. D'après le théorème $2.7(2), D^{\prime}$ est une $G$-variété.

Lemme 3.6. Soit $G$ un groupe linéaire connexe. Alors $\operatorname{Br}_{G}(G)=\operatorname{Br}_{1}(G)$.

Démonstration. D'après la proposition 3.4 (2) et (4), il suffit de montrer que $\operatorname{Br}_{G}\left(G_{\bar{k}}\right)=0$ et on peut supposer $k=\bar{k}$.

Si $G$ est un tore de dimension $n$, alors $G \cong \mathbb{G}_{m}^{n}$ est un ouvert de $\mathbb{A}^{n}$ canoniquement. Soit $X:=\mathbb{A}^{n} \backslash\left[\left(\mathbb{A}^{n} \backslash G\right)_{\text {sing }}\right]$. Alors $\operatorname{codim}\left(\mathbb{A}^{n} \backslash X, \mathbb{A}^{n}\right) \geq 2, \operatorname{Br}(X)=0, G$ est un ouvert de $X$ et $X \backslash G=\sqcup_{i=1}^{n} D_{i}$, chaque $D_{i}$ étant un $G$-espace homogène de stabilisateur $\mathbb{G}_{m}$. D'après [CX1, Prop. 2.2], pour tout revêtement fini étale galoisien $D_{i}^{\prime} \stackrel{\pi_{i}}{\rightarrow} D_{i}$ tel que $D_{i}^{\prime}$ soit une $G$-variété intègre et que $\pi_{i}$ soit un $G$-morphisme, le morphisme $\pi_{i}$ est un isomorphisme. D'après le lemme 3.5 (2) et $(3.3), \operatorname{Br}_{G}(G) \subset \operatorname{Br}(X)=0$.

Si $G$ est réductif, soit $T$ le tore maximal de $G$. Par la décomposition de Bruhat, il existe un ouvert $U$ de $G$ tel que $U \cong \mathbb{A}^{n} \times T \times \mathbb{A}^{n}$, où $2 n=\operatorname{dim}(U)-\operatorname{dim}(T)$ (voir la démonstration de [CT-Beijing, Prop. 4.2]). Donc $\operatorname{Br}(G) \rightarrow \operatorname{Br}(T)$ est injectif. Le résultat découle de la proposition $3.4(2)$.

En général, par [CDX, Lem. 2.1], le morphisme $\operatorname{Br}\left(G^{\text {red }}\right) \rightarrow \operatorname{Br}(G)$ est un isomorphisme. Le résultat découle de la proposition 3.4 (2).

Rappelons la définition de $\operatorname{Br}_{e}(G)$ (cf. (1.2)).

Proposition 3.7. Soient $G$ un groupe linéaire connexe et $(X, \rho)$ une G-variété lisse géométriquement intègre. Pour tout $b \in \operatorname{Br}_{G}(X)$, on a $\left(\rho^{*}(b)-p_{2}^{*}(b)\right) \in p_{1}^{*} \operatorname{Br}_{e}(G)$.

Démonstration. Notons $X \stackrel{e_{G} \times i d_{X}}{\longrightarrow} G \times X$. Puisque $p_{2} \circ\left(e_{G} \times i d_{X}\right)=\rho \circ\left(e_{G} \times i d_{X}\right)$, il suffit de montrer que, pour tout $b \in \operatorname{Br}_{G}(X)$, on a $\left(\rho^{*}(b)-p_{2}^{*}(b)\right) \in p_{1}^{*} \operatorname{Br}_{1}(G)$. 
On peut supposer $k=\bar{k}$. Un point $x \in X(k)$ induit un morphisme $G \stackrel{i_{x}}{\rightarrow} X$. Notons $m$ la multiplication sur $G$. Alors on a un diagramme commutatif :

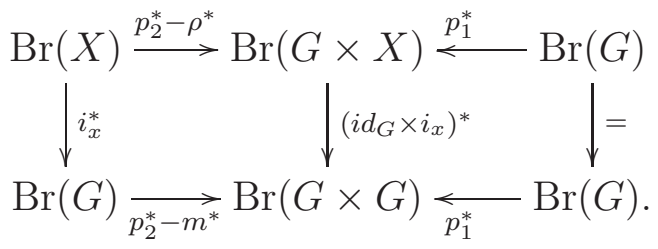

Le résultat découle du lemme 3.6 et de l'injectivité de $p_{1}^{*}$.

3.2. L'homomorphisme de Sansuc. Soient $G$ un groupe algébrique connexe et $(X, \rho)$ une $G$-variété lisse géométriquement intègre. Notons $G \times X \stackrel{p_{1}}{\rightarrow} G, G \times X \stackrel{p_{2}}{\longrightarrow} X$ les deux projections. Soit $\operatorname{Br}_{G}(X)$ le sous-groupe de Brauer $G$-invariant de $X$ (Définition 3.1). Rappelons la définition de $\operatorname{Br}_{e}(G)$ (cf. (1.2)).

Par [S, Lem. 6.6], on a $\operatorname{Br}_{a}(G \times X)=\operatorname{Br}_{a}(G) \oplus \operatorname{Br}_{a}(X)$, et donc $\left.p_{1}^{*}\right|_{\mathrm{Br}_{e}(G)}$ est injectif. Par la proposition 3.7, il existe un unique homomorphisme $\operatorname{Br}_{G}(X) \stackrel{\lambda}{\rightarrow} \operatorname{Br}_{e}(G)$ tel que

$$
p_{1}^{*} \circ \lambda=\rho^{*}-p_{2}^{*}: \operatorname{Br}_{G}(X) \rightarrow \operatorname{Br}(G \times X) .
$$

Définition 3.8. Soient $G$ un groupe linéaire connexe et $(X, \rho)$ une $G$-variété lisse géométriquement intègre. L'unique homomorphisme $\operatorname{Br}_{G}(X) \stackrel{\lambda}{\rightarrow} \operatorname{Br}_{e}(G)$ satisfaisant (3.4) est appelé l'homomorphisme de Sansuc.

Proposition 3.9. Sous les hypothèses de la définition 3.8, on a :

(1) pour toute extension de corps $K / k$, et tous $x \in X(K), g \in G(K), \alpha \in \operatorname{Br}_{G}(X)$, on a

$$
(g \cdot x)^{*}(\alpha)=g^{*}(\lambda(\alpha))+x^{*}(\alpha) \in \operatorname{Br}(K)
$$

(2) si $X(k) \neq \emptyset$, alors pour tout $x \in X(k)$, on a

$$
\lambda=\rho_{x}^{*}-x^{*},
$$

où $G \stackrel{\rho_{x}}{\rightarrow} X: g \mapsto g \cdot x$, Spec $k \stackrel{x}{\rightarrow} X$ est le point $x$ et $\operatorname{Br}(X) \stackrel{x^{*}}{\rightarrow} \operatorname{Br}(k) \subset \operatorname{Br}(G)$.

(3) si $X \cong G / G_{0}$ avec $G_{0} \subset G$ un sous-groupe fermé connexe, alors

$$
\operatorname{Br}_{G}(X) \cong \operatorname{Br}_{1}(X, G):=\operatorname{Ker}\left(\operatorname{Br}(X) \rightarrow \operatorname{Br}\left(G_{\bar{k}}\right)\right)
$$

Démonstration. Pour (1), on a

$$
(g \cdot x)^{*}(\alpha)=(g, x)^{*}\left(\rho^{*}(\alpha)\right)=(g, x)^{*}\left(p_{2}^{*}(\alpha)+p_{1}^{*}(\lambda(\alpha))\right)=g^{*}(\lambda(\alpha))+x^{*}(\alpha) \in \operatorname{Br}(K) .
$$

Dans le cas (2), pour tout $\alpha \in \operatorname{Br}_{G}(X)$, on obtient $\left.\left(\rho^{*}-p_{2}^{*}\right)(\alpha)\right|_{G \times x}=\left(\rho_{x}^{*}-x^{*}\right)(\alpha)$.

L'énoncé (3) résulte de la proposition $3.4(2)$ (5) et du lemme 3.6.

Si $X \stackrel{f}{\rightarrow} Z$ est un $G$-torseur, par la définition, l'homomorphisme de Sansuc $\left.\lambda\right|_{\operatorname{Br}_{1}(X)}$ est exactement le morphisme dans la suite exacte de Sansuc (cf : [BD, Thm. 2.8]).

Théorème 3.10. Soient $G$ un groupe linéaire connexe, $Z$ une variété lisse géométriquement intègre et $X \stackrel{f}{\rightarrow} Z$ un $G$-torseur. Notons $G \times X \stackrel{\rho}{\rightarrow} X$ l'action de $G$. Alors l'homomorphisme de 
Sansuc $\lambda$ induit un diagramme commutatif de suites exactes :

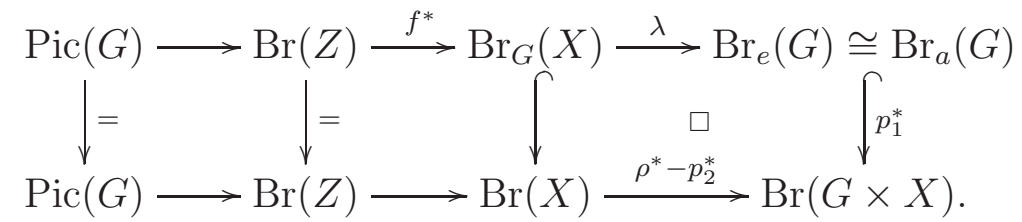

Démonstration. D'après Borovoi et Demarche [BD, Thm. 2.8], on a une suite exacte

$$
\operatorname{Pic}(G) \rightarrow \operatorname{Br}(Z) \stackrel{f^{*}}{\rightarrow} \operatorname{Br}(X) \stackrel{\rho^{*}-p_{2}^{*}}{\longrightarrow} \operatorname{Br}(G \times X)
$$

telle que $\left(\rho^{*}-p_{2}^{*}\right)\left(\operatorname{Br}_{1}(X)\right) \subset p_{1}^{*} \operatorname{Br}_{e}(G)$. Le résultat découle de la proposition 3.7.

Corollaire 3.11. Soient $1 \rightarrow N \rightarrow H \stackrel{\psi}{\rightarrow} G \rightarrow 1$ une suite de groupes linéaires connexes et $(X, \rho)$ une $G$-variété lisse géométriquement intègre.

(1) On a $\mathrm{Br}_{G}(X)=\mathrm{Br}_{H}(X)$.

(2) S'il existe une $H$-variété $Y$ et un $H$-morphisme $Y \stackrel{p}{\rightarrow} X$ tels que $Y \rightarrow X$ soit un $N$ torseur, alors $\operatorname{Br}(X) \stackrel{p^{*}}{\rightarrow} \operatorname{Br}(Y)$ satisfait $p^{*} \operatorname{Br}_{G}(X) \subset \operatorname{Br}_{H}(Y),\left(p^{*}\right)^{-1} \operatorname{Br}_{H}(Y)=\operatorname{Br}_{G}(X)$ et on a une suite exacte (où $\lambda$ est l'homomorphisme de Sansuc) :

$$
\operatorname{Pic}(N) \rightarrow \operatorname{Br}_{G}(X) \stackrel{p^{*}}{\rightarrow} \operatorname{Br}_{H}(Y) \stackrel{\lambda}{\rightarrow} \operatorname{Br}_{a}(N)
$$

Démonstration. On a $\operatorname{Br}_{1}(H) \subset \operatorname{Br}_{N}(H)$ et $H \times X \stackrel{\psi_{X}}{\longrightarrow} G \times X$ est un $N$-torseur. Par la suite exacte de Sansuc ([S, Prop. 6.10]) et le diagramme (3.6), on a un diagramme commutatif de suites exactes :

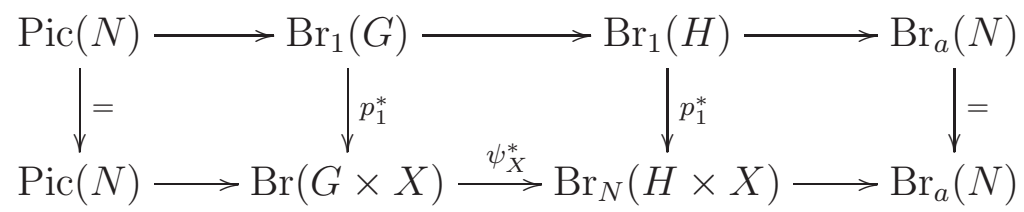

Donc $\left(\psi_{X}^{*}\right)^{-1}\left(p_{1}^{*} \operatorname{Br}_{e}(H)\right)=p_{1}^{*} \operatorname{Br}_{e}(G)$ et on obtient (1).

Une application de la proposition $3.4(5)$ et du diagramme (3.6) donne (2).

Lemme 3.12. Soient $G, N$ deux groupes linéaires connexes et $X$ une $G$-variété lisse géométriquement intègre. Soient $H:=N \times G$ et $P$ une $H$-variété telle que $P$ soit un $N$-torseur sur $k$. Soient $Y \stackrel{\sim}{\rightarrow} P \times X$ et $Y \stackrel{p_{1}}{\rightarrow} P, Y \stackrel{p_{2}}{\rightarrow} X$ les deux projections. Si $P(k) \neq \emptyset$ ou $H^{3}\left(k, \bar{k}^{\times}\right)=0$, on a un isomorphisme:

$$
\operatorname{Br}_{a}(P) \oplus \operatorname{Br}_{G}(X) / \operatorname{ImBr}(k) \stackrel{\left(p_{1}^{*}, p_{2}^{*}\right)}{\longrightarrow} \operatorname{Br}_{H}(Y) / \operatorname{ImBr}(k) .
$$

De plus, cet isomorphisme induit un isomorphisme : $\operatorname{Br}_{a}(P) \oplus \operatorname{Br}_{a}(X) \stackrel{\left(p_{1}^{*}, p_{2}^{*}\right)}{\longrightarrow} \operatorname{Br}_{a}(Y)$.

Démonstration. Par la proposition 3.4 (1) et le lemme 3.6, on a $\operatorname{Br}_{H}(P)=\operatorname{Br}_{1}(P)$. Par la suite exacte de Sansuc ([S, Prop. 6.10]) et le corollaire 3.11 (2), on a un diagramme commutatif de 
suites exactes

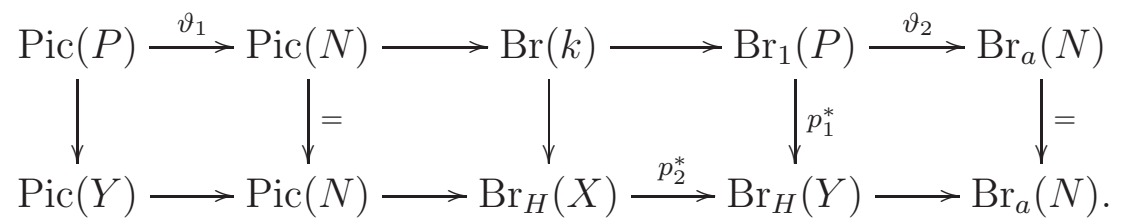

Par [S, Lem. 6.7 et 6.8], $\vartheta_{1}$ et $\vartheta_{2}$ sont surjectifs. Alors on a une suite exacte

$$
0 \rightarrow \operatorname{Br}(k) \rightarrow \operatorname{Br}_{1}(P) \oplus \operatorname{Br}_{H}(X) \stackrel{\left(p_{1}^{*}, p_{2}^{*}\right)}{\longrightarrow} \operatorname{Br}_{H}(Y) \rightarrow 0 .
$$

Puisque le morphisme $\operatorname{Br}\left(X_{\bar{k}}\right) \rightarrow \operatorname{Br}\left((P \times X)_{\bar{k}}\right) \cong \operatorname{Br}\left(Y_{\bar{k}}\right)$ est injectif, on a une suite exacte :

$$
0 \rightarrow \operatorname{Br}(k) \rightarrow \operatorname{Br}_{1}(P) \oplus \operatorname{Br}_{1}(X) \stackrel{\left(p_{1}^{*}, p_{2}^{*}\right)}{\longrightarrow} \operatorname{Br}_{1}(Y) \rightarrow 0 .
$$

Le résultat en découle.

Proposition 3.13. Soient $T$ un tore et $1 \rightarrow G_{0} \rightarrow G \stackrel{\psi}{\rightarrow} T \rightarrow 1$ une suite exacte de groupes linéaires connexes. Soient $X$ une $G$-variété lisse, géométriquement intègre et $X \stackrel{f}{\rightarrow} T$ un $G$ morphisme. Notons $\operatorname{Br}_{a}(G) \stackrel{\vartheta}{\rightarrow} \operatorname{Br}_{a}\left(G_{0}\right)$ l'homomorphisme induit par $G_{0} \subset G$. Alors, pour tout $t \in T(k)$, la fibre $X_{t}$ est $G_{0}$-invariante et on a un isomorphisme naturelle $\operatorname{Pic}\left(X_{\bar{k}}\right) \cong \operatorname{Pic}\left(X_{t, \bar{k}}\right)$ et deux suites exactes naturelles

$$
0 \rightarrow T^{*} \stackrel{f^{*}}{\rightarrow} \bar{k}[X]^{\times} / \bar{k}^{\times} \rightarrow \bar{k}\left[X_{t}\right]^{\times} / \bar{k}^{\times} \rightarrow 0 \quad \text { et } \operatorname{Br}_{e}(T) \rightarrow \operatorname{Br}_{G}(X) \rightarrow \operatorname{Br}_{G_{0}}\left(X_{t}\right) \rightarrow \operatorname{coker}(\vartheta) .
$$

Démonstration. D’après [CX1, Prop. 2.2], $X_{t}$ est lisse, géométriquement intègre. Notons :

$$
X_{t} \stackrel{i}{\rightarrow} G \times X_{t}: x \mapsto\left(e_{G}, x\right) \text { et } G \times X_{t} \stackrel{\rho}{\rightarrow} X:(g, x) \mapsto g \cdot x .
$$

Alors $\rho \circ i$ est l'immersion $X_{t} \subset X$. On fixe des actions

$G \times G_{0} \curvearrowright G \times X_{t}:\left(g, g_{0}\right) \times\left(g^{\prime}, x\right) \mapsto\left(g g^{\prime} g_{0}^{-1}, g_{0} \cdot x\right)$ et $G \times G_{0} \curvearrowright G:\left(g, g_{0}\right) \times g^{\prime} \mapsto g g^{\prime} g_{0}^{-1}$.

Par définition, $X \cong G \times{ }^{G_{0}} X_{t}$ et on a un diagramme commutatif de $G \times G_{0}$-morphismes

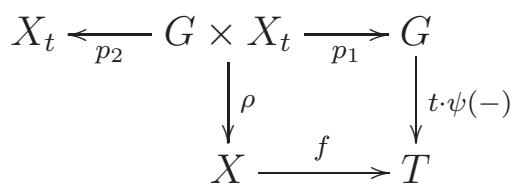

tel que les colonnes soient des $G_{0}$-torseurs.

D'après [S, Lem. 6.5 et Lem. 6.6], on a

$$
\bar{k}\left[G \times X_{t}\right]^{\times} / \bar{k}^{\times} \cong \bar{k}[G]^{\times} / \bar{k}^{\times} \oplus \bar{k}\left[X_{t}\right]^{\times} / \bar{k}^{\times} \quad \text { et } \operatorname{Pic}\left(G_{\bar{k}} \times X_{t, \bar{k}}\right) \cong \operatorname{Pic}\left(G_{\bar{k}}\right) \oplus \operatorname{Pic}\left(X_{t, \bar{k}}\right) \text {. }
$$

Par la suite exacte de Sansuc [S, Prop. 6.10 et Cor. 6.11], on a un diagramme commutatif de suites exactes :

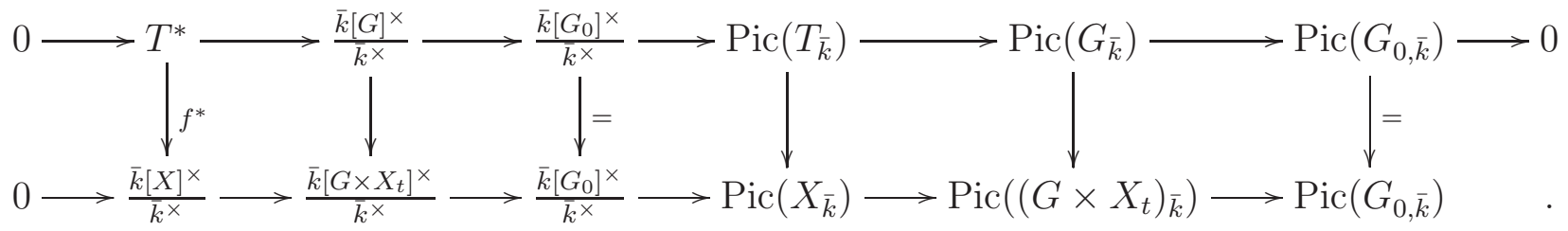


Puisque $\operatorname{Pic}\left(T_{\bar{k}}\right)=0$, une application du lemme du serpent donne l'isomorphisme et la première suite exacte de l'énoncé.

D'après (3.7), on a un isomorphisme : $\operatorname{Br}_{e}(G) \oplus \operatorname{Br}_{G_{0}}\left(X_{t}\right) \stackrel{\left(p_{1}^{*}, p_{2}^{*}\right)}{\longrightarrow} \operatorname{Br}_{G \times G_{0}}\left(G \times X_{t}\right)$. Le corollaire 3.11 donne un diagramme commutatif de suites exactes :

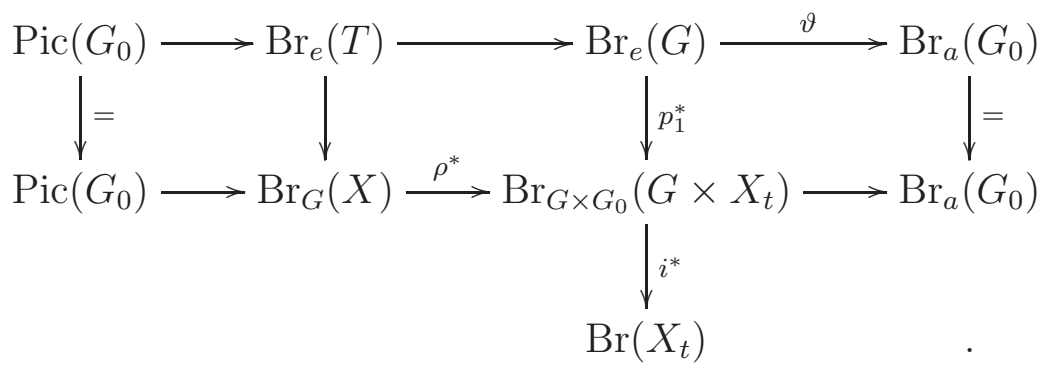

Puisque $p_{2} \circ i=i d$ et $i^{*} \circ p_{1}^{*}=0$, on a $\operatorname{Br}_{G_{0}}\left(X_{t}\right)=\operatorname{Im}\left(i^{*}\right) \cong \operatorname{coker}\left(p_{1}^{*}\right)$. Une chasse au diagramme donne l'énoncé.

Corollaire 3.14. Sous les hypothèses de la proposition 3.13, soient $U \subset X$ un $G$-ouvert et $B \subset \operatorname{Br}_{G}(U)$ un sous-groupe. Alors, pour tout $t \in T(k)$, de fibre $U_{t} \subset X_{t} \stackrel{i_{t}}{\longrightarrow} X$, on $a$ :

$$
i_{t}^{*}(B \cap \operatorname{Br}(X))=\left(i_{t}^{*}(B) \cap \operatorname{Br}\left(X_{t}\right)\right) .
$$

Démonstration. D'après la proposition 3.13, on a un diagramme de suites exactes :

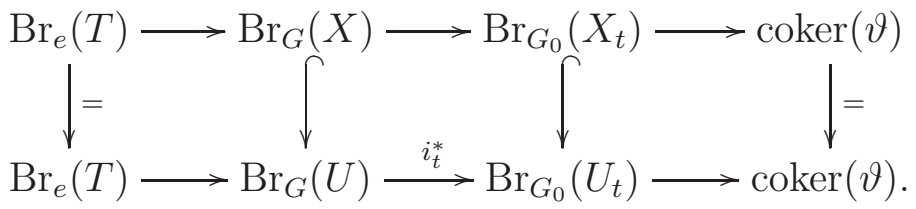

Une chasse au diagramme donne l'énoncé.

3.3. Pseudo espace homogène. Soit $G$ un groupe linéaire connexe. La notion de pseudo $G$-espace homogène généralise la notion de $G$-espace homogène à stabilisateur géométrique connexe (cf. exemple $3.16(1)$ ).

Définition 3.15. Soit $G$ un groupe linéaire connexe. Une $G$-variété $Z$ est appelée pseudo $G$ espace homogène si $Z$ est lisse, géométriquement intègre, $\operatorname{Pic}\left(Z_{\bar{k}}\right)$ est de type fini, $Z(k) \neq \emptyset$ et $\frac{\operatorname{Ker}(\lambda)}{\operatorname{Br}(k)}, \operatorname{Br}_{G_{\bar{k}}}\left(Z_{\bar{k}}\right)$ sont finis, où $\operatorname{Br}_{G}(Z) \stackrel{\lambda}{\rightarrow} \operatorname{Br}_{e}(G)$ est l'homomorphisme de Sansuc (cf. Définition $3.8)$.

Fixons $z \in Z(k)$ et notons $\rho_{z}: G \rightarrow Z: g \mapsto g \cdot z$. D'après (3.5), le groupe $\frac{\operatorname{Ker}(\lambda)}{\operatorname{Br}(k)}$ est fini si et seulement si $\operatorname{Ker}\left(\rho_{z}^{*}\right) \cap \operatorname{Br}_{G}(Z)$ est fini, où $\operatorname{Br}(Z) \stackrel{\rho_{z}^{*}}{\longrightarrow} \operatorname{Br}(G)$.

Example 3.16. Soit $G$ un groupe linéaire connexe.

(1) Soit $G_{0} \subset G$ un sous-groupe fermé connexe. Alors $G / G_{0}$ est un pseudo $G$-espace homogène.

(2) Soient $X$ une variété lisse géométriquement intègre et $Z \rightarrow X$ un $G$-torseur. $\operatorname{Si} \operatorname{Pic}(X)$ est de type fini, $\operatorname{Br}(X) / \operatorname{Br}(k), \operatorname{Br}\left(X_{\bar{k}}\right)$ sont finis et $Z(k) \neq \emptyset$, alors $Z$ est un pseudo $G$-espace homogène. 
Démonstration. L'énoncé (1) résulte de [BD, Thm. 2.8] et de la proposition 3.9 (3). L'énoncé (2) résulte du corollaire 3.11 (2).

Proposition 3.17. Soient $G$ un groupe linéaire connexe et $Z$ un pseudo G-espace homogène. Soient $T$ un tore, $Z^{\prime} \rightarrow Z$ un $T$-torseur et $H$ le groupe linéaire connexe déterminé dans le théorème 2. '. Si $Z^{\prime}(k) \neq \emptyset$, alors $Z^{\prime}$ est un pseudo $H$-espace homogène.

Démonstration. Fixons $z^{\prime} \in Z^{\prime}(k)$ et $z \in Z(k)$ l'image de $z$. Notons $\rho_{z^{\prime}}: H \rightarrow Z^{\prime}: h \mapsto h \cdot z^{\prime}$ et $\rho_{z}: G \rightarrow Z: g \mapsto g \cdot z$. D'après la suite exacte de Sansuc [S, Prop. 6.10], $\operatorname{Pic}\left(Z^{\prime}{ }_{k}\right)$ est de type fini. Par le corollaire 3.11 (2), on a un diagramme commutatif de suites exactes :

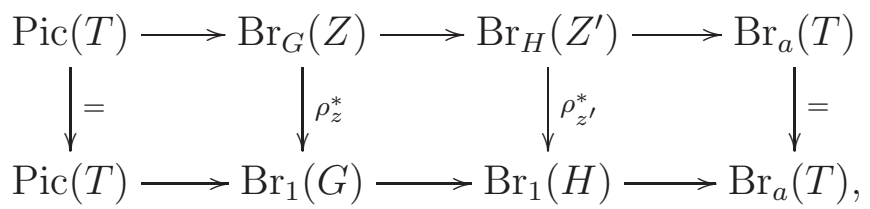

et un isomorphisme $\operatorname{Br}_{G_{\bar{k}}}\left(Z_{\bar{k}}\right) \rightarrow \operatorname{Br}_{H_{\bar{k}}}\left(Z_{\bar{k}}^{\prime}\right)$. Ainsi $\operatorname{Br}_{H_{\bar{k}}}\left(Z_{\bar{k}}^{\prime}\right)$ est fini. Puisque $\operatorname{Ker}\left(\rho_{z}^{*}\right)$ est fini, le groupe $\operatorname{Ker}\left(\rho_{z^{\prime}}^{*}\right)$ est fini et $Z^{\prime}$ est un pseudo $H$-espace homogène.

Proposition 3.18. Soient $G$ un groupe linéaire connexe et $Z$ un pseudo G-espace homogène. Alors $\operatorname{Br}_{G}(Z) / \operatorname{Br}_{1}(Z)$ est fini.

Démonstration. Ceci vaut car $\operatorname{Br}_{G}(Z) / \operatorname{Br}_{1}(Z)$ est un sous-groupe de $\operatorname{Br}_{G_{\bar{k}}}\left(Z_{\bar{k}}\right)$.

Pour un corps de nombres, le lemme suivant est bien connu.

Lemme 3.19. Supposons que $k$ est un corps de nombres. Alors :

(i) pour tout $\Gamma_{k}$-module de type fini sans torsion $M \neq 0$, le groupe $H^{2}(k, M)$ est infini;

(ii) pour tout $\Gamma_{k}$-module fini $M \neq 0$, le groupe $H^{1}(k, M)$ est infini.

Lemme 3.20. Supposons que $k$ est un corps de nombres. Soit $\phi: M \rightarrow N$ un homomorphisme de $\Gamma_{k}$-modules de type fini sans torsion. Si $\operatorname{Ker}\left(H^{2}(k, M) \stackrel{\phi_{*}}{\rightarrow} H^{2}(k, N)\right)$ est fini, alors $\phi$ est injectif et $\operatorname{coker}(\phi)$ est sans torsion.

Démonstration. Notons $I:=\operatorname{Im}(\phi), K:=\operatorname{Ker}(\phi)$ et $C:=\operatorname{coker}(\phi)$. Alors $I$ et $K$ sont de type fini sans torsion et on a deux suites exactes :

$$
H^{1}(k, I) \rightarrow H^{2}(k, K) \rightarrow H^{2}(k, M) \stackrel{\theta_{1}}{\rightarrow} H^{2}(k, I)
$$

et

$$
H^{1}(k, N) \rightarrow H^{1}(k, C) \rightarrow H^{2}(k, I) \stackrel{\theta_{2}}{\rightarrow} H^{2}(k, N) .
$$

Ainsi $H^{1}(k, I), H^{1}(k, N)$ et $\operatorname{Ker}\left(\theta_{1}\right)$ sont finis. Alors $H^{2}(k, K)$ est fini et donc $K=0$ (Lemme 3.19). Ainsi $\operatorname{Ker}\left(\theta_{2}\right)$ est fini. Alors $H^{1}(k, C)$ est fini et donc $C$ est sans torsion (Lemme 3.19).

Lemme 3.21. Supposons que $k$ est un corps de nombres. Soient $G$ un groupe linéaire connexe, $T$ un tore et $G \stackrel{\psi}{\rightarrow} T$ un homomorphisme. Alors les énoncés suivants sont équivalents :

(i) le groupe $\operatorname{Ker}\left(\operatorname{Br}_{1}(T) \stackrel{\psi^{*}}{\longrightarrow} \operatorname{Br}_{1}(G)\right)$ est fini;

(ii) le morphisme $\psi$ est surjectif de noyau connexe;

(iii) la G-variété $T$ est un pseudo G-espace homogène. 
Démonstration. D'après l'exemple 3.16 (1), on a (ii) $\Rightarrow$ (iii). Par définition, on a (iii) $\Rightarrow($ i). Pour (i) $\Rightarrow$ (ii), par la suite exacte de Sansuc, on peut remplacer $G$ par $G^{\text {tor }}$ et supposer que $G$ est un tore. Dans ce cas, le noyau de $H^{2}\left(k, T^{*}\right) \stackrel{\psi^{*}}{\longrightarrow} H^{2}\left(k, G^{*}\right)$ est fini. Le lemme 3.20 ci-dessus donne (ii).

Soient $G$ un groupe linéaire connexe et $Z$ un pseudo $G$-espace homogène (cf. Définition 3.15). Soient $\left(Z^{\text {tor }}\right)^{*}:=\bar{k}[Z]^{\times} / \bar{k}^{\times}$un $\Gamma_{k^{-}}$module libre de type fini et $Z^{\text {tor }}$ le tore correspondant. Pour tout $z \in Z(k)$, on a un morphisme canonique $Z \stackrel{\pi_{z}}{\longrightarrow} Z^{\text {tor }}$ tel que $\pi_{z}(z)=e_{Z^{\text {tor }}}$ (Rosenlicht). Soit $\psi_{z}$ la composition $G \rightarrow G \cdot z \hookrightarrow Z \stackrel{\pi_{z}}{\rightarrow} Z^{\text {tor }}$.

Proposition 3.22. Le morphisme $\psi_{z}$ ne dépend pas du choix de z, et c'est un homomorphisme tel que $Z^{\text {tor }}$ soit une $G$-variété et $\pi_{z}$ soit un $G$-morphisme.

De plus, si $k$ est un corps de nombres, la $G$-variété $Z^{\text {tor }}$ est un pseudo $G$-espace homogène.

Démonstration. Notons $\rho: G \times Z \rightarrow Z$ l'action de $G$. Par le lemme de Rosenlicht, $\psi_{z}$ est un homomorphisme. D'après le lemme 2.6, on a un isomorphisme canonique

$$
H_{e_{G}}^{0}\left(G, Z^{\text {tor }}\right) \oplus H^{0}\left(Z, Z^{\text {tor }}\right) \underset{\theta}{\stackrel{p_{1}^{*} \cdot p_{2}^{*}}{\rightleftarrows}} H^{0}\left(G \times Z, Z^{\text {tor }}\right): \quad\left(\psi_{z}, \pi_{z}\right) \longmapsto \psi_{z} \cdot \pi_{z}
$$

tel que $\theta\left(\pi_{z} \circ \rho\right)=\left(\left.\left(\pi_{z} \circ \rho\right)\right|_{G \times z},\left.\left(\pi_{z} \circ \rho\right)\right|_{e_{G} \times Z}\right)=\left(\psi_{z}, \pi_{z}\right)$. Alors $\pi_{z} \circ \rho=\psi_{z} \cdot \pi_{z}$ et $\pi_{z}$ est un $G$-morphisme.

Pour tout $z^{\prime} \in Z(k)$, on a $\pi_{z^{\prime}}=\pi_{z}\left(z^{\prime}\right) \cdot \pi_{z}$ et donc $\theta\left(\pi_{z^{\prime}} \circ \rho\right)=\left(\psi_{z}, \pi_{z}\left(z^{\prime}\right) \cdot \pi_{z}\right)$. Ainsi $\psi_{z^{\prime}}=\psi_{z}$.

La suite spectrale de Hochschild-Serre donne une suite exacte

$$
\operatorname{Pic}(Z) \rightarrow \operatorname{Pic}\left(Z_{\bar{k}}\right)^{\Gamma_{k}} \rightarrow \operatorname{Br}_{1}\left(Z^{\text {tor }}\right) \stackrel{\left.\pi_{z}^{*}\right|_{\mathrm{Br}_{1}}}{\longrightarrow} \operatorname{Br}(Z) .
$$

Puisque $\operatorname{Pic}\left(Z_{\bar{k}}\right)$ est de type fini et $\operatorname{Br}_{1}\left(Z^{\text {tor }}\right)$ est torsion, le groupe $\operatorname{Ker}\left(\left.\pi_{z}^{*}\right|_{\mathrm{Br}_{1}}\right)$ est fini. Puisque $\operatorname{Ker}\left(\operatorname{Br}_{G}(Z) \stackrel{\rho_{z}^{*}}{\longrightarrow} \operatorname{Br}(G)\right)$ est fini, le groupe $\operatorname{Ker}\left(\operatorname{Br}_{1}\left(Z^{\text {tor }}\right) \stackrel{\left.\psi_{z}^{*}\right|_{\operatorname{Br}}}{\longrightarrow} \operatorname{Br}(G)\right)$ est fini. Si $k$ est un corps de nombres, une application du lemme 3.21 donne l'énoncé.

Définition 3.23. Dans la proposition 3.22, une fois qu'on a choisi $z \in Z(k)$, le morphisme $Z \stackrel{\pi}{\rightarrow} Z^{\text {tor }}$ est appelé le quotient torique maximal. Il ne dépend du choix de $z$ qu'à translation près. Si $k$ est un corps de nombres, le morphisme $G \rightarrow Z^{\text {tor }}$ dans la proposition 3.22 est surjectif de noyau $G_{0}$ connexe. Le groupe $G_{0}$ est appelé le stabilisateur de $G$ sur $Z^{\text {tor }}$.

\section{L'APPRoximation FORTE HORS DES PlaCES ARChimédiennes ET LA QUESTION 1.2}

Dans toute cette section, $k$ est un corps de nombres. Sauf mention explicite, une variété est une $k$-variété. Soit $G$ un groupe linéaire connexe. Pour répondre à la question 1.2, on établit le théorème 4.2. Comme consequence, on montre le théorème 1.4 (1).

Rappelons la notion de sous-groupe de Brauer invariant (cf. Définition 3.1).

Lemme 4.1. Soit $G$ un groupe linéaire connexe. Alors l'homomorphisme induit par l'accouplement de Brauer-Manin $G\left(\mathbf{A}_{k}\right) . \stackrel{\theta_{G}}{\longrightarrow} \operatorname{Br}_{a}(G)^{D}$ est ouvert, où $(-)^{D}:=\operatorname{Hom}(-, \mathbb{Q} / \mathbb{Z})$.

Démonstration. Dans $G\left(\mathbf{A}_{k}\right)$, les sous-groupes ouverts compacts forment une base topologique de $e_{G}$. Pour tout tel sous-groupe $C$, l'image $\theta_{G}(C) \subset \operatorname{Br}_{a}(G)^{D}$ est compacte, et donc fermée. Il suffit alors de montrer que cette image est d'indice fini. Par la finitude de nombre de classes de 
$G$ ([PR, Thm. 5.1]), il existe un tel sous-groupe $C_{0}$ tel que la classe double $C_{0} \backslash G\left(\mathbf{A}_{k}\right) \bullet / G(k)$ soit finie. Puisque $\amalg^{1}(G)$ est fini, d'après [D11, Thm. 5.1], $\theta_{G}\left(C_{0}\right)$ est d'indice fini. Pour tout tel sous-groupe $C$, le quotient $C_{0} /\left(C \cap C_{0}\right)$ est fini et donc $\theta_{G}(C)$ est d'indice fini.

Théorème 4.2. Soient $G$ un groupe linéaire connexe, $X$ une $G$-variété lisse géométriquement intègre et $U \subset X$ un $G$-ouvert. Soient $A \subset \operatorname{Br}(X)$ un sous-groupe fini et $B \subset \operatorname{Br}_{G}(U)$ (cf. (3.1)) un sous-groupe. Supposons que $\frac{B \cap \operatorname{Ker}(\lambda)}{B \cap \operatorname{ImBr}(k)}$ est fini, où $\operatorname{Br}_{G}(U) \stackrel{\lambda}{\rightarrow} \operatorname{Br}_{e}(G)$ est l'homomorphisme de Sansuc (Définition 3.8). Alors, pour tout ouvert $W \subset X\left(\mathbf{A}_{k}\right)$ satisfaisant $W^{(A+B) \cap B r(X)} \neq \emptyset$, on $a W \cap U\left(\mathbf{A}_{k}\right)^{A+B} \neq \emptyset$.

Démonstration. On peut supposer que $\operatorname{ImBr}(k) \subset B$. Après avoir rétréci $W$, on peut supposer que tout élément de $A$ s'annule sur $W$ et $W \cong W_{\infty} \times W_{f}$ avec $W_{\infty} \subset X\left(k_{\infty}\right), W_{f} \subset X\left(\mathbf{A}_{k}^{\infty}\right)$ tel que $W_{f}$ soit compact.

Pour tout $w \in W_{f}$, il existe un ouvert $W_{w} \subset W_{f}$ contenant $w$ et un ouvert $C_{w} \subset G\left(\mathbf{A}^{\infty}\right)$ tel que $C_{w} \cdot W_{w} \subset W_{f}$. Puisque $W_{f}$ est compact, il existe un sous-ensemble fini $I \subset W_{f}$ tel que $\cup_{w \in I} W_{w}=W_{f}$. Soit $C_{f}$ le sous-groupe de $G\left(\mathbf{A}^{\infty}\right)$ engendré par $\cap_{w \in I} C_{w}$. Alors $C_{f} \cdot W_{f} \subset W_{f}$ et $C_{f}$ est ouvert dans $G\left(\mathbf{A}^{\infty}\right)$. Soit $C:=\left(e_{G}\right)_{\infty} \times C_{f} \subset G\left(\mathbf{A}_{k}\right)$. Alors $C \cdot W=W$ et l'image de $C$ dans $G\left(\mathbf{A}_{k}\right)$. est ouvert.

Notons $G\left(\mathbf{A}_{k}\right) \stackrel{\theta_{G}}{\longrightarrow} \operatorname{Br}_{a}(G)^{D}$ et $U\left(\mathbf{A}_{k}\right) \stackrel{\theta_{U}}{\longrightarrow} B^{D}$ les applications induites par l'accouplement de Brauer-Manin, où $(-)^{D}:=\operatorname{Hom}(-, \mathbb{Q} / \mathbb{Z})$. D'après le lemme 4.1, $\theta_{G}(C) \subset \operatorname{Br}_{a}(G)^{D}$ est un sous-groupe ouvert d'indice fini. Par hypothèse, il existe un sous-groupe fini $B_{1} \subset B$ tel que

$$
\operatorname{Ker}\left((B)^{D} \stackrel{\vartheta}{\rightarrow}\left(B_{1}+\operatorname{ImBr}(k)\right)^{D}\right) \subset\left(\lambda^{D} \circ \theta_{G}\right)(C),
$$

où $\vartheta$ est induit par l'inclusion $B_{1}+\operatorname{ImBr}(k) \subset B$ et l'homomorphisme

$$
\lambda: B \subset \operatorname{Br}_{G}(U) \rightarrow \operatorname{Br}_{e}(G)=\operatorname{Br}_{a}(G) \text { induit } \lambda^{D}:=\operatorname{Hom}(\lambda, \mathbb{Q} / \mathbb{Z}): \operatorname{Br}_{a}(G)^{D} \rightarrow B^{D} .
$$

D'après le lemme formel de Harari ([Ha94, Cor. 2.6.1]), $W \cap U\left(\mathbf{A}_{k}\right)^{B_{1}} \neq \emptyset$. On a un diagramme :

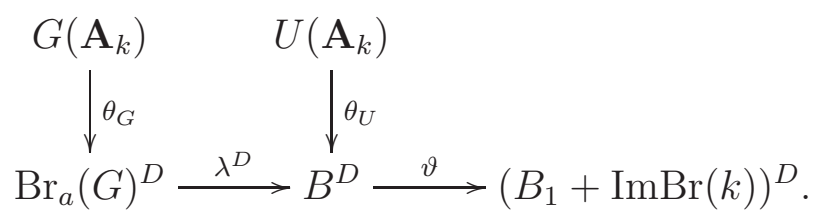

Soit $u \in W \cap U\left(\mathbf{A}_{k}\right)^{B_{1}}$, alors $\vartheta\left(\theta_{U}(u)\right)=0$ et, d'après (4.1), il existe $g \in C$ tel que $g^{-1} \cdot u \in W$ et $\left(\lambda^{D} \circ \theta_{G}\right)(g)=\theta_{U}(u)$. D'après la proposition $3.9(1)$, on a $\theta_{U}\left(g^{-1} \cdot u\right)=0$.

Remarque 4.3. Dans le théorème 4.2, si $G=1$, alors ce théorème est équivalent au lemme formel de Harari ([Ha94, Cor. 2.6.1]).

Comme conséquence directe, on a :

Corollaire 4.4. Avec les hypothèses et notations du théorème 4.2, pour tout sous-ensemble fini $S \subset \Omega_{k}$, s'il existe un ouvert $X_{1}$ de $X$ tel que $U \subset X_{1}$ et $X_{1}$ satisfasse l'approximation forte par rapport à $\operatorname{Br}\left(X_{1}\right) \cap(A+B)$ hors de $S$, alors $X$ satisfait l'approximation forte par rapport $\grave{a} \operatorname{Br}(X) \cap(A+B)$ hors de $S$. 
Corollaire 4.5. Avec les hypothèses et notations du théorème 4.2, s'il existe un ouvert $X_{1}$ de $X$ tel que $U \subset X_{1}$ et $X_{1}(k)$ soit dense dans $X_{1}\left(\mathbf{A}_{k}\right)_{\bullet}^{\operatorname{Br}\left(X_{1}\right) \cap(A+B)}$, alors $X(k)$ est dense dans $X\left(\mathbf{A}_{k}\right)_{\bullet}^{\operatorname{Br}(X) \cap(A+B)}$.

Soit $G$ un groupe linéaire connexe. Rappelons la notion de pseudo $G$-espace homogène (cf. Définition 3.15).

Théorème 4.6. Soient $G$ un groupe linéaire connexe et $Z$ un pseudo $G$-espace homogène. Soient $X$ une $G$-variété lisse, géométriquement intègre et $U \subset X$ un $G$-ouvert muni d'un $G$-morphisme $U \stackrel{f}{\rightarrow} Z$. Soient $A \subset \operatorname{Br}(X)$ et $B \subset \operatorname{Br}_{G}(U)$ (cf. (3.1)) deux sous-groupes finis. Alors, pour tout ouvert $W \subset X\left(\mathbf{A}_{k}\right)$ satisfaisant $W^{\operatorname{Br}(X) \cap\left(A+B+f^{*} \operatorname{Br}_{G}(Z)\right)} \neq \emptyset$,

(1) on a $W \cap U\left(\mathbf{A}_{k}\right)^{A+B+f^{*} \operatorname{Br}_{G}(Z)} \neq \emptyset$;

(2) si $G\left(k_{\infty}\right)^{+} \cdot Z(k)$ est dense dans $Z\left(\mathbf{A}_{k}\right)^{\operatorname{Br}_{G}(Z)}$, il existe $z \in Z(k)$ de fibre $U_{z}$ tel que

$$
\left(G\left(k_{\infty}\right)^{+} \cdot W\right) \cap U_{z}\left(\mathbf{A}_{k}\right)^{A+B} \neq \emptyset .
$$

Démonstration. Notons $\operatorname{Br}_{G}(Z) \stackrel{\lambda_{Z}}{\longrightarrow} \operatorname{Br}_{a}(G), \operatorname{Br}_{G}(U) \stackrel{\lambda_{U}}{\longrightarrow} \operatorname{Br}_{a}(G)$ les homomorphismes de Sansuc (cf. Définition 3.8). Puisque $\operatorname{Ker}\left(\lambda_{Z}\right) / \operatorname{ImBr}(k)$ est fini et $\lambda_{Z}=\lambda_{U} \circ f^{*}$, le quotient $\frac{\operatorname{Ker}\left(\lambda_{U}\right) \cap\left(B+f^{*} \operatorname{Br}_{G}(Z)\right)}{\operatorname{ImBr}(k)}$ est fini. Une application du théorème 4.2 donne $(1)$.

D'après [Cod, Thm. 4.5], l'application $U\left(\mathbf{A}_{k}\right) \rightarrow Z\left(\mathbf{A}_{k}\right)$ est ouverte et on obtient (2).

Démonstration du théorème 1.4 (1). Par la proposition 3.9 (3) et l'approximation forte pour les espaces homogènes à stabilisateur géométrique connexe (Borovoi et Demarche [BD, Thm. 1.4]), $U(k)$ est dense dans $U\left(\mathbf{A}_{k}\right)^{\operatorname{Br}_{G}(U)}$. Une application du théorème 4.6 (2) donne l'énoncé.

\section{LA DESCENTE PAR RAPPORT AU GROUPE DE BRAUER INVARIANT}

Dans toute cette section, $k$ est un corps de nombres. Sauf mention explicite du contraire, une variété est une $k$-variété. La méthode de descente des points adéliques est établie par Colliot-Thélène et Sansuc dans [CTSb]. Dans [CDX], C. Demarche, F. Xu et l'auteur étudient la méthode de descente des points adéliques orthogonaux à certains groupes de Brauer dans le cas des torseurs sous un tore. On suit leur méthode et considère ici le cas plus général des torseurs sous un groupe linéaire connexe (Théorème 5.9).

Rappelons la notion de sous-groupe de Brauer invariant (cf. Définition 3.1).

5.1. Torseur sous un groupe linéaire dont le groupe de Shafarevich est trivial. Soient $G$ un groupe linéaire connexe, $X$ une variété lisse géométriquement intègre et $Y \stackrel{p}{\rightarrow} X$ un $G$ torseur. Pour tout $\sigma \in H^{1}(k, G)$, soient $P_{\sigma}$ le $G$-torseur correspondant et $G_{\sigma}$ le tordu de $G$ correspondant. Alors $P_{\sigma}$ est un $G_{\sigma}$-torseur et une $\left(G_{\sigma} \times G\right)$-variété. Soit $Y_{\sigma} \stackrel{p_{\sigma}}{\longrightarrow} X$ le tordu de $[Y]$, i.e. $Y_{\sigma}=P_{\sigma} \times^{G} Y$. Alors $\left[Y_{\sigma}\right]$ est un $G_{\sigma}$-torseur sur $X$. Soit $\operatorname{Br}_{G_{\sigma}}\left(Y_{\sigma}\right)$ le sous-groupe de Brauer $G_{\sigma}$-invariant de $Y_{\sigma}$ (Définition 3.1).

Lemme 5.1. Supposons que $\amalg^{1}(G)=1$. Alors pour tout sous-groupe $B \subset \operatorname{Br}_{G}(Y)$, on $a$ :

$$
p\left(Y\left(\mathbf{A}_{k}\right)^{\left(p^{*} \circ\left(p^{*}\right)^{-1}\right) B}\right)=p\left(Y\left(\mathbf{A}_{k}\right)^{B}\right),
$$

où $\operatorname{Br}(X) \stackrel{p^{*}}{\rightarrow} \operatorname{Br}(Y)$. 
Démonstration. Puisque $\left(p^{*} \circ\left(p^{*}\right)^{-1}\right) B \subset B$, on a $p\left(Y\left(\mathbf{A}_{k}\right)^{B}\right) \subset p\left(Y\left(\mathbf{A}_{k}\right)^{\left(p^{*} \circ\left(p^{*}\right)^{-1}\right) B}\right)$.

Par le théorème 3.10 , on a un diagramme commutatif de suites exactes

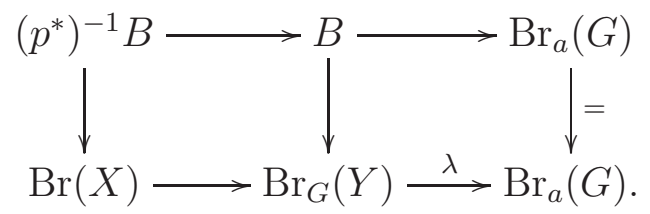

Il induit un diagramme commutatif avec suite exacte :

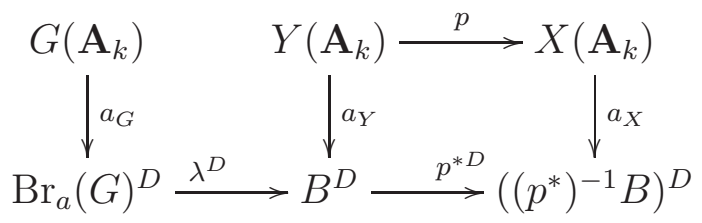

où $(-)^{D}:=\operatorname{Hom}(-, \mathbb{Q} / \mathbb{Z})$ et $a_{G}, a_{Y}, a_{X}$ sont induits par l'accouplement de Brauer-Manin. Par la proposition 3.9, pour chaque $y \in Y\left(\mathbf{A}_{k}\right)$ et $g \in G\left(\mathbf{A}_{k}\right)$, on a $a_{Y}(g \cdot y)=\left(\lambda^{D} \circ a_{G}\right)(g)+a_{Y}(y)$.

Puisque $\amalg^{1}(G)=1$, par la suite exacte de Poitou-Tate de $G$ (Demarche [D11, Thm. 5.1]), $a_{G}$ est surjectif. Pour tout $y \in p\left(Y\left(\mathbf{A}_{k}\right)^{\left(p^{*} \circ\left(p^{*}\right)^{-1}\right) B}\right)$, on a $x:=p(y) \in X\left(\mathbf{A}_{k}\right)^{\left(p^{*}\right)^{-1} B}$. Alors $\left(p^{* D} \circ a_{Y}\right)(y)=a_{X}(x)=0$ et il existe $g \in G\left(\mathbf{A}_{k}\right)$ tel que $\left(\lambda^{D} \circ a_{G}\right)(g)=a_{Y}(y)$. Donc $a_{Y}\left(g^{-1} \cdot y\right)=$ 0 et $p\left(g^{-1} \cdot y\right)=x$.

Proposition 5.2. Soient $G$ un groupe linéaire connexe, $X$ une variété lisse géométriquement intègre et $Y \stackrel{p}{\rightarrow} X$ un $G$-torseur. Soit $A \subset \operatorname{Br}(X)$ un sous-groupe et, pour chaque $\sigma \in H^{1}(k, G)$, soit $B_{\sigma} \subset \operatorname{Br}_{G_{\sigma}}\left(Y_{\sigma}\right)$ un sous-groupe. Supposons que $\amalg^{1}(G)=1$ et que, pour tout $\sigma \in H^{1}(k, G)$, on $a\left(p_{\sigma}^{*}\right)^{-1}\left(B_{\sigma}\right) \subset A$, où $\operatorname{Br}(X) \stackrel{p_{\sigma}^{*}}{\longrightarrow} \operatorname{Br}\left(Y_{\sigma}\right)$. Alors on $a$ :

$$
X\left(\mathbf{A}_{k}\right)^{A}=\cup_{\sigma \in H^{1}(k, G)} p_{\sigma}\left(Y_{\sigma}\left(\mathbf{A}_{k}\right)^{B_{\sigma}+p_{\sigma}^{*} A}\right) .
$$

Démonstration. Le résultat découle du [CDX, Thm. 1.1] et du lemme 5.1.

Corollaire 5.3. Soit $T$ un tore quasi-trivial. Soient $X$ une variété lisse, géométriquement intègre et $Y \stackrel{p}{\rightarrow} X$ un $T$-torseur. Soient $U \subset X$ un ouvert et $V=p^{-1}(U)$. Alors pour tous sous-groupes $A \subset \operatorname{Br}(U), B \subset \operatorname{Br}_{T}(V)$, si $\left(p^{*}\right)^{-1}(B) \subset A$, où $\operatorname{Br}(U) \stackrel{p^{*}}{\rightarrow} \operatorname{Br}(V)$, on a

$$
X\left(\mathbf{A}_{k}\right)^{\operatorname{Br}(X) \cap A}=p\left(Y\left(\mathbf{A}_{k}\right)^{\operatorname{Br}(Y) \cap\left(B+p^{*} A\right)}\right) .
$$

Démonstration. Par le lemme 3.2, on a $\left(p^{*}\right)^{-1}\left(\operatorname{Br}(Y) \cap\left(p^{*} A+B\right)\right)=\operatorname{Br}(X) \cap A$. D'après la proposition 3.4, on a $\left(\operatorname{Br}(Y) \cap\left(p^{*} A+B\right)\right) \subset \operatorname{Br}_{T}(Y)$. L'énoncé résulte de la proposition 5.2.

5.2. L'application de la résolution coflasque. Par [CTSa], un $\Gamma_{k}$-module $M$ de type fini est appelé coflasque si $M$ est sans torsion et, pour tout sous-groupe fermé $\Gamma \subset \Gamma_{k}$, on a $H^{1}(\Gamma, M)=0$. Un $k$-tore $T$ est appelé coflasque si $T^{*}$ est coflasque. Alors $H^{1}\left(k, T^{*}\right)=0$ et, pour tout $v \in \Omega_{k}$, on a $H^{1}\left(k_{v}, T^{*}\right)=0$. Dans [CTSa, Prop. 1.3], il y a des résolutions par les tores coflasques et les tores quasi-triviaux.

Lemme 5.4. Soit $T$ un tore. Alors $H^{3}\left(k, T^{*}\right) \cong \prod_{v \in \infty_{k}} H^{3}\left(k_{v}, T^{*}\right) \cong \prod_{v \in \infty_{k}} H^{1}\left(k_{v}, T^{*}\right)$. 
Démonstration. Pour tout $v \in \infty_{k}$, on a $H^{3}\left(k_{v}, T^{*}\right)=H^{1}\left(k_{v}, T^{*}\right)$. Par la ligne 3 de la démonstration de [HS05, Prop. 5.9], on a $H^{3}\left(k, T^{*}\right)=\prod_{v \in \infty_{k}} H^{3}\left(k_{v}, T^{*}\right)$. Le résultat en découle.

Lemme 5.5. Soit

$$
1 \rightarrow G \stackrel{\psi}{\rightarrow} H \stackrel{\phi}{\rightarrow} T \rightarrow 1
$$

une suite exacte de groupes linéaires connexes avec $T$ un tore. Supposons que $H^{3}\left(k, T^{*}\right)=0$. Alors le morphisme $\mathrm{Br}_{a}(H) \stackrel{\psi^{*}}{\rightarrow} \operatorname{Br}_{a}(G)$ est surjectif.

Démonstration. Par la suite exacte de Sansuc [S, Cor. 6.11], on a une suite exacte de $\Gamma_{k}$-modules

$$
0 \rightarrow T^{*} \rightarrow H^{*} \rightarrow G^{*} \rightarrow 0
$$

et un isomorphisme de $\Gamma_{k}$-modules $\operatorname{Pic}\left(H_{\bar{k}}\right) \rightarrow \operatorname{Pic}\left(G_{\bar{k}}\right)$. Par [CTX09, Lem. 2.1], on a un diagramme commutatif de suites exactes :

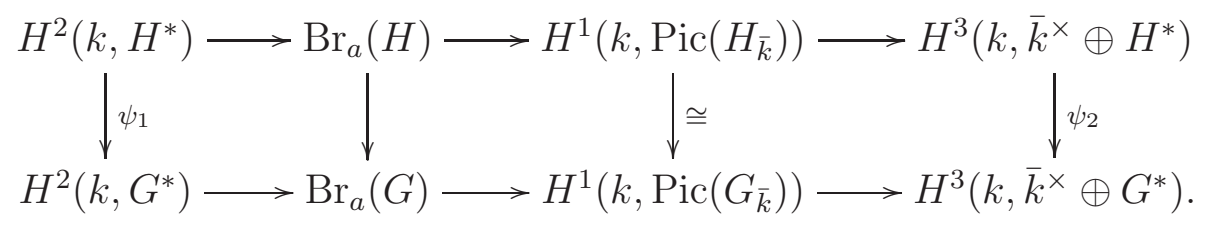

Puisque $H^{3}\left(k, T^{*}\right)=0$, le morphisme $\psi_{1}$ est surjectif et le morphisme $\psi_{2}$ est injectif. Donc le morphisme $\mathrm{Br}_{a}(H) \rightarrow \mathrm{Br}_{a}(G)$ est surjectif.

Proposition 5.6. Soit $G$ un groupe réductif connexe. Alors il existe un groupe linéaire connexe $H$, un tore $T$ et une suite exacte:

$$
1 \rightarrow G \stackrel{\psi}{\rightarrow} H \stackrel{\phi}{\rightarrow} T \rightarrow 1
$$

tels que $\amalg^{1}(H)=0, H^{3}\left(k, T^{*}\right)=0$ et $\operatorname{Br}_{a}(H) \stackrel{\psi^{*}}{\rightarrow} \operatorname{Br}_{a}(G)$ soit surjectif.

De plus, si $G$ est un tore, on peut imposer que $H$ soit un tore quasi-trivial.

Démonstration. Puisque $G$ est réductif, il existe une suite exacte $0 \rightarrow S \rightarrow G \rightarrow G^{a d} \rightarrow 0$ telle que $S$ soit le centre de $G$ et $G^{a d}$ soit le groupe adjoint de $G$. Alors $S$ est un groupe de type multiplicatif. Par [CTSa, Prop. 1.3], il existe un tore quasi-trivial $T_{0}$ et un homomorphisme injectif $S \stackrel{\chi}{\rightarrow} T_{0}$ tels que $T:=T_{0} / S$ soit un tore coflasque. Alors, pour tout $v \in \infty_{k}$, on a $H^{1}\left(k_{v}, T^{*}\right)=0$. Par le lemme 5.4, on a $H^{3}\left(k, T^{*}\right)=0$.

Soit $H:=G \times{ }^{S} T_{0}$. Alors $H$ est un groupe linéaire et on a deux suites exactes

$$
1 \rightarrow G \stackrel{\psi}{\rightarrow} H \stackrel{\phi}{\rightarrow} T \rightarrow 1 \text { et } 1 \rightarrow T_{0} \rightarrow H \rightarrow G^{a d} \rightarrow 1 .
$$

Par le lemme 5.5, le morphisme $\operatorname{Br}_{a}(H) \stackrel{\psi^{*}}{\rightarrow} \operatorname{Br}_{a}(G)$ est surjectif. Par [S, Cor. 5.4], on a $\amalg^{1}\left(G^{a d}\right)=0$. Puisque $T_{0}$ est quasi-trivial, on a $\amalg^{1}(H)=0$.

Proposition 5.7. Soit $X$ une variété lisse géométriquement intègre. Supposons que $X(k) \neq \emptyset$ et que $\operatorname{Pic}\left(X_{\bar{k}}\right)$ est de type fini. Alors il existe un tore quasi-trivial $T$ et un $T$-torseur $Y \rightarrow X$ tels que $\operatorname{Pic}\left(Y_{\bar{k}}\right)=0$ et $H^{3}\left(k, \bar{k}[Y]^{\times} / \bar{k}^{\times}\right)=0$.

Démonstration. Il existe un ouvert $U \subset X$ tel que $\operatorname{Pic}\left(U_{\bar{k}}\right)=0$. Soient $T_{0}$ un tore tel que $T_{0}^{*}:=\operatorname{Div}_{X_{\bar{k}} \backslash U_{\bar{k}}}\left(X_{\bar{k}}\right)$ et $Y_{0} \rightarrow X$ le $T_{0}$-torseur induit par l'homomorphisme $\Psi$ de la suite exacte (2.2). Par la suite exacte de Sansuc [S, Prop. 6.10], $\operatorname{Pic}\left(Y_{0, \bar{k}}\right)=0$. Soit $T_{1}$ le tore tel que 
$T_{1}^{*} \cong \bar{k}\left[Y_{0}\right]^{\times} / \bar{k}^{\times}$. Puisque $X(k) \neq \emptyset$, on a $Y_{0}(k) \neq \emptyset$ et il induit un morphisme $Y_{0} \stackrel{\pi}{\rightarrow} T_{1}$ tel que $T_{1}^{*} \stackrel{\pi^{*}}{\rightarrow} \bar{k}\left[Y_{0}\right]^{\times} / \bar{k}^{\times}$soit un isomorphisme.

D'après [CTSa, Prop. 1.3], il existe une suite exacte $0 \rightarrow T_{2} \rightarrow T_{3} \rightarrow T_{1} \rightarrow 0$ telle que $T_{2}$ soit quasi-trivial et que $T_{3}$ soit coflasque. Soit $Y:=Y_{0} \times_{T_{1}} T_{3}$ un $T_{2}$ torseur sur $Y_{0}$. Par la suite exacte de Sansuc [S, Prop. 6.10], $\operatorname{Pic}\left(Y_{\bar{k}}\right)=0, \bar{k}[Y]^{\times} / \bar{k}^{\times}=T_{3}^{*}$ et donc $H^{3}\left(k, \bar{k}[Y]^{\times} / \bar{k}^{\times}\right)=0$.

Soit $T:=T_{0} \times T_{2}$. Puisque $T_{0}, T_{2}$ sont quasi-triviaux, on a $H^{1}\left(T_{0}, T_{2}\right)=0$ et, d'après [CT08, Cor. 5.7], toute extension centrale de $T_{0}$ par $T_{2}$ est isomorphe à $T_{0} \times T_{2}$. D'après le théorème 2.7, il existe une action de $T$ sur $Y$ compatible avec les actions $T_{2}$ sur $Y$ et $T_{0}$ sur $Y_{0}$. Alors $Y$ est un $T$-torseur sur $X$.

5.3. La descente générale. Soient $G$ un groupe linéaire connexe, $X$ une variété lisse géométriquement intègre et $Y \stackrel{p}{\rightarrow} X$ un $G$-torseur. Pour tout $\sigma \in H^{1}(k, G)$, soient $P_{\sigma}$ le $G$-torseur correspondant et $G_{\sigma}$ le tordu de $G$ correspondant. Alors $P_{\sigma}$ est un $G_{\sigma}$-torseur et une $\left(G_{\sigma} \times G\right)$ variété. Soit $Y_{\sigma} \stackrel{p_{\sigma}}{\longrightarrow} X$ le tordu de $[Y]$, i.e. $Y_{\sigma}=P_{\sigma} \times^{G} Y$. Alors $\left[Y_{\sigma}\right]$ est un $G_{\sigma}$-torseur sur $X$. Soit $\operatorname{Br}_{G_{\sigma}}\left(Y_{\sigma}\right)$ le sous-groupe de Brauer $G_{\sigma}$-invariant de $Y_{\sigma}$ (Définition 3.1).

Notons $P_{\sigma} \times Y \stackrel{\theta_{Y}^{\sigma}}{\longrightarrow} Y_{\sigma}$. Le lemme 3.12 donne un isomorphisme canonique :

$$
\operatorname{Br}_{a}\left(P_{\sigma}\right) \oplus \operatorname{Br}_{G}(Y) / \operatorname{ImBr}(k) \stackrel{\left(p_{1}^{*}, p_{2}^{*}\right)}{\longrightarrow} \operatorname{Br}_{G_{\sigma} \times G}\left(P_{\sigma} \times Y\right) / \operatorname{ImBr}(k) .
$$

Il induit un morphisme canonique

$$
\Theta_{Y}^{\sigma}: \operatorname{Br}_{G_{\sigma}}\left(Y_{\sigma}\right) / \operatorname{ImBr}(k) \stackrel{\left(\theta_{Y}^{\sigma}\right)^{*}}{\longrightarrow} \operatorname{Br}_{G_{\sigma} \times G}\left(P_{\sigma} \times Y\right) / \operatorname{ImBr}(k) \rightarrow \operatorname{Br}_{G}(Y) / \operatorname{ImBr}(k) .
$$

Lemme 5.8. Notons $\sigma^{-1}:=\left[P_{\sigma}\right] \in H^{1}\left(k, G_{\sigma}\right)$. Alors $Y \cong\left(Y_{\sigma}\right)_{\sigma^{-1}}$ et $\Theta_{Y}^{\sigma}$ est un isomorphisme d'inverse $\Theta_{Y_{\sigma}}^{\left(\sigma^{-1}\right)}$.

Démonstration. On a $\left(Y_{\sigma}\right)_{\sigma^{-1}}=P_{\sigma} \times{ }^{G_{\sigma}} P_{\sigma} \times{ }^{G} Y \cong G \times{ }^{G} Y \cong Y$. On a un isomorphisme canonique $P_{\sigma} \times Y \stackrel{p_{1} \times \theta_{Y}^{\sigma}}{\longrightarrow} P_{\sigma} \times Y_{\sigma}$ d'inverse $p_{1} \times \theta_{Y_{\sigma}}^{\left(\sigma^{-1}\right)}$. Par le lemme 3.12, on a :

$$
\operatorname{Br}_{a}\left(P_{\sigma}\right) \oplus \operatorname{Br}_{G}(Y) / \operatorname{ImBr}(k) \cong \operatorname{Br}_{G_{\sigma} \times G}\left(P_{\sigma} \times Y\right) / \operatorname{ImBr}(k)
$$

et

$$
\operatorname{Br}_{a}\left(P_{\sigma}\right) \oplus \operatorname{Br}_{G_{\sigma}}\left(Y_{\sigma}\right) / \operatorname{ImBr}(k) \cong \operatorname{Br}_{G_{\sigma} \times G}\left(P_{\sigma} \times Y_{\sigma}\right) / \operatorname{ImBr}(k) .
$$

Le résultat en découle.

Pour un sous-groupe $B_{\sigma} \subset \operatorname{Br}_{G_{\sigma}}\left(Y_{\sigma}\right)$ contenant $\operatorname{ImBr}(k)$, on note $\widetilde{\Theta_{Y}^{\sigma}}\left(B_{\sigma}\right) \subset \operatorname{Br}_{G}(Y)$ l'image inverse de $\Theta_{Y}^{\sigma}\left(B_{\sigma}\right)$. Alors $\widetilde{\Theta_{Y_{\sigma}}^{\left(\sigma^{-1}\right)}}\left(\widetilde{\Theta_{Y}^{\sigma}}\left(B_{\sigma}\right)\right)=B_{\sigma}$.

Théorème 5.9. Soient $G$ un groupe linéaire connexe, $X$ une variété lisse géométriquement intègre et $Y \stackrel{p}{\rightarrow} X$ un $G$-torseur. Soit $A \subset \operatorname{Br}(X)$ un sous-groupe et, pour chaque $\sigma \in H^{1}(k, G)$, soit $B_{\sigma} \subset \operatorname{Br}_{G_{\sigma}}\left(Y_{\sigma}\right)$ un sous-groupe contenant $\operatorname{ImBr}(k)$. Supposons que, pour tout $\sigma \in H^{1}(k, G)$, on $a$

$$
\left(p_{\sigma}^{*}\right)^{-1}\left(\sum_{\sigma^{\prime} \in \amalg^{1}\left(G_{\sigma}\right)} \widetilde{\Theta_{Y_{\sigma}}^{\sigma^{\prime}}}\left(B_{\sigma+\sigma^{\prime}}\right)\right) \subset A
$$

où $\operatorname{Br}(X) \stackrel{p_{\sigma}^{*}}{\longrightarrow} \operatorname{Br}\left(Y_{\sigma}\right)$ et $Y_{\sigma+\sigma^{\prime}}:=\left(Y_{\sigma}\right)_{\sigma^{\prime}}$. Alors on a :

$$
X\left(\mathbf{A}_{k}\right)^{A}=\cup_{\sigma \in H^{1}(k, G)} p_{\sigma}\left(Y_{\sigma}\left(\mathbf{A}_{k}\right)^{B_{\sigma}+p_{\sigma}^{*} A}\right) .
$$


Démonstration. Par [CDX, Thm. 1.1], il suffit de montrer que, pour tout $\sigma \in H^{1}(k, G)$, on a :

$$
p_{\sigma}\left(Y_{\sigma}\left(\mathbf{A}_{k}\right)^{p_{\sigma}^{*} A}\right) \subset \cup_{\sigma^{\prime} \in \amalg^{1}\left(G_{\sigma}\right)} p_{\sigma+\sigma^{\prime}}\left(Y_{\sigma+\sigma^{\prime}}\left(\mathbf{A}_{k}\right)^{B_{\sigma+\sigma^{\prime}}+p_{\sigma+\sigma^{\prime}}^{*} A}\right) .
$$

On peut supposer que $\sigma=0$. Pour tout $v$, puisque $H^{1}\left(k_{v}, G^{u}\right)=0$, le morphisme $Y\left(k_{v}\right) \rightarrow$ $\left(G^{\text {red }} \times{ }^{G} Y\right)\left(k_{v}\right)$ est surjectif. Puisque $\amalg^{1}(G) \cong \amalg^{1}\left(G^{\text {red }}\right)$ ([S, Prop. 4.1]) et que l'on a $\operatorname{Br}(Y) \cong$ $\operatorname{Br}\left(G^{\text {red }} \times{ }^{G} Y\right)$ ([CDX, Lem. 2.1]), on peut supposer que $G$ est réductif.

Par la proposition 5.6, il existe un groupe linéaire connexe $H$, un tore $T$ et une suite exacte :

$$
1 \rightarrow G \stackrel{\psi}{\rightarrow} H \stackrel{\phi}{\rightarrow} T \rightarrow 1
$$

tels que $\amalg^{1}(H)=0, H^{3}\left(k, T^{*}\right)=0$ et $\operatorname{Br}_{a}(H) \stackrel{\psi^{*}}{\rightarrow} \operatorname{Br}_{a}(G)$ soit surjectif. Par [Se65, Prop. $36 \mathrm{du}$ Chapitre 1], on a une suite exacte d'ensembles pointés :

$$
1 \rightarrow G(k) \rightarrow H(k) \rightarrow T(k) \stackrel{\partial}{\rightarrow} H^{1}(k, G) \rightarrow H^{1}(k, H),
$$

telle que, pour tout $t \in T$, on ait $\partial(t):=\left[\phi^{-1}(t)\right]$. Alors $\amalg^{1}(G) \subset \operatorname{Im}(\partial)$ et, pour tout $t \in T(k)$, on a $G_{\partial(t)} \cong G$.

Soient $Y_{H}:=H \times{ }^{G} Y$ et $Y \stackrel{i}{\rightarrow} Y_{H}$ le morphisme canonique. Alors $Y_{H} \stackrel{p_{H}}{\rightarrow} X$ est un $H$-torseur sur $X$ et $T \times^{H} Y_{H} \cong T \times X$. Alors on a un morphisme canonique $Y_{H} \stackrel{\mu}{\rightarrow} T$ tel que, pour tout $t \in T(k)$, on a $\mu^{-1}(t) \cong \phi^{-1}(t) \times{ }^{G} Y \cong Y_{\partial(t)}$. Notons $\mu^{-1}(t) \stackrel{i_{t}}{\rightarrow} Y_{H}$. Par la définition de $\Theta_{Y}^{\partial(t)}$, on a $: \Theta_{Y}^{\partial(t)} \circ i_{t}^{*}=i^{*}$. Par la proposition 3.13 et le lemme 5.8, les morphismes $\operatorname{Br}_{H}\left(Y_{H}\right) \stackrel{i^{*}}{\rightarrow} \operatorname{Br}_{G}(Y)$ et $\operatorname{Br}_{H}\left(Y_{H}\right) \stackrel{i_{t}^{*}}{\rightarrow} \operatorname{Br}_{G}\left(\mu^{-1}(t)\right)$ sont surjectifs.

Notons

$$
B:=\operatorname{Br}_{H}\left(Y_{H}\right) \cap\left(i^{*}\right)^{-1}\left(\sum_{\sigma \in \amalg^{1}(G)} \widetilde{\Theta_{Y}^{\sigma}}\left(B_{\sigma}\right)\right) \subset \operatorname{Br}_{H}\left(Y_{H}\right) .
$$

Alors $\left(p_{H}^{*}\right)^{-1}(B) \subset A, B_{\partial(t)} \subset i_{t}^{*} B$ et $\mu^{*} \operatorname{Br}_{1}(T) \subset B$.

Pour un $y \in Y\left(\mathbf{A}_{k}\right)^{p^{*} A}$, par le lemme 5.1, il existe $y_{1} \in Y_{H}\left(\mathbf{A}_{k}\right)^{B+p_{H}^{*} A}$ tel que $p_{H}\left(y_{1}\right)=p(y)$. Alors il existe $h \in H\left(\mathbf{A}_{k}\right)$ tel que $y_{1}=h \cdot y$. Donc $\mu\left(y_{1}\right) \in \phi\left(H\left(\mathbf{A}_{k}\right)\right) \cap T\left(\mathbf{A}_{k}\right)^{\operatorname{Br}_{1}(T)}$.

Par [CDX, Prop. 3.2], on a :

$$
T\left(\mathbf{A}_{k}\right)^{\operatorname{Br}_{1}(T)}=T(k) \cdot \phi\left(H\left(\mathbf{A}_{k}\right)^{\operatorname{Br}_{1}(H)}\right) .
$$

Donc il existe $h_{1} \in H\left(\mathbf{A}_{k}\right)^{\operatorname{Br}_{1}(H)}$ tel que

$$
t:=\mu\left(h_{1} \cdot y_{1}\right) \in T(k) \cap \phi\left(H\left(\mathbf{A}_{k}\right)\right) .
$$

Donc $\partial(t) \subset \amalg^{1}(G)$. Par la proposition $3.9(1)$,

$$
y_{2}:=h_{1} \cdot y_{1} \in Y_{H}\left(\mathbf{A}_{k}\right)^{B+p_{H}^{*} A} \quad \text { et donc } \quad y_{2} \in \mu^{-1}(t)\left(\mathbf{A}_{k}\right)^{B_{\partial(t)}+p_{\partial(t)}^{*}}{ }^{A} .
$$

Le résultat découle de $p_{\partial(t)}\left(y_{2}\right)=p_{H}\left(y_{2}\right)=p(y)$.

Corollaire 5.10. Sous les hypothèses du théorème 5.9, soit

$$
X(k) \stackrel{\partial}{\rightarrow} H^{1}(k, G): z \mapsto\left[p^{-1}(z)\right]
$$

le morphisme canonique. Supposons que pour tout $\sigma \in H^{1}(k, G)$, la variété $Y_{\sigma}$ satisfait le principe de Hasse par rapport à $B_{\sigma}+p_{\sigma}^{*} A$. Alors

$$
X\left(\mathbf{A}_{k}\right)^{A}=\cup_{\sigma \in \operatorname{Im}(\partial)} p_{\sigma}\left(Y_{\sigma}\left(\mathbf{A}_{k}\right)^{B_{\sigma}+p_{\sigma}^{*} A}\right) .
$$


Démonstration. Pour tout $\sigma \in H^{1}(k, G)$, si $Y_{\sigma}\left(\mathbf{A}_{k}\right)^{B_{\sigma}+p_{\sigma}^{*} A} \neq \emptyset$, alors on a $Y_{\sigma}(k) \neq \emptyset$ et donc $\sigma \in \operatorname{Im}(\partial)$. Le résultat en découle.

Corollaire 5.11. Soient $G$ un groupe linéaire connexe, $X$ une variété lisse géométriquement intègre et $Y \stackrel{p}{\rightarrow} X$ un $G$-torseur. Soit $\operatorname{Br}_{1}(X, Y):=\operatorname{Ker}\left(\operatorname{Br}(X) \rightarrow \operatorname{Br}\left(Y_{\bar{k}}\right)\right)$. Alors on a :

$$
X\left(\mathbf{A}_{k}\right)^{\operatorname{Br}_{1}(X, Y)}=\cup_{\sigma \in H^{1}(k, G)} p_{\sigma}\left(Y_{\sigma}\left(\mathbf{A}_{k}\right)^{\operatorname{Br}_{1}\left(Y_{\sigma}\right)}\right)
$$

Démonstration. Pour tout $\sigma \in H^{1}(k, G)$, puisque le morphisme $\operatorname{Br}\left(Y_{\bar{k}}\right) \rightarrow \operatorname{Br}\left(\left(P_{\sigma} \times Y\right)_{\bar{k}}\right)$ est injectif, on a $\operatorname{Br}_{1}\left(X, Y_{\sigma}\right) \subset \operatorname{Br}_{1}(X, Y)$. Donc $\operatorname{Br}_{1}\left(X, Y_{\sigma}\right)=\operatorname{Br}_{1}(X, Y)=\left(p^{*}\right)^{-1} \operatorname{Br}_{1}(Y)$.

Pour tout $\sigma \in H^{1}(k, G)$, par le lemme 3.12 et le lemme 5.8, le morphisme $\Theta_{Y}^{\sigma}$ induit un isomorphisme $\operatorname{Br}_{a}\left(Y_{\sigma}\right) \rightarrow \operatorname{Br}_{a}(Y)$. Donc $\widetilde{\Theta_{Y}^{\sigma}}\left(\operatorname{Br}_{1}\left(Y_{\sigma}\right)\right)=\operatorname{Br}_{1}(Y)$. Le résultat découle du théorème 5.9 .

Remarque 5.12. La démonstration du théorème 5.9 n'utilise pas l'approximation forte pour les espaces homogènes à stabilisateur géométrique connexe (Borovoi et Demarche [BD, Thm. $1.4])$.

Corollaire 5.13. Soient $G$ un groupe linéaire connexe, $G_{0} \subset G$ un sous groupe fermé connexe et $Z:=G / G_{0}$. Notons $G \stackrel{\pi}{\rightarrow} Z$ la projection. Alors on a

$$
Z\left(\mathbf{A}_{k}\right)^{\operatorname{Br}_{G}(Z)}=\pi\left(G\left(\mathbf{A}_{k}\right)^{\operatorname{Br}_{1}(G)}\right) \cdot Z(k) .
$$

Démonstration. Puisque $G \stackrel{\pi}{\rightarrow} Z$ est un $G_{0}$-torseur à droite, pour tout $P_{\sigma} \in H^{1}\left(k, G_{0}\right)$, le tordu $G \times{ }^{G_{0}} P_{\sigma}$ est un $G$ torseur à gauche. Donc $G \times{ }^{G_{0}} P_{\sigma}$ satisfait le principe de Hasse par rapport à $\operatorname{Br}_{1}\left(G \times{ }^{G_{0}} P_{\sigma}\right)$ (Sansuc, cf. [Sko01, Thm. 5.2.1]). Le résultat découle du corollaire 5.10.

\section{La méthode de Fibration et LA QUESTION 1.3}

Dans toute cette section, $k$ est un corps de nombres. Sauf mention explicite du contraire, une variété est une $k$-variété. Pour traiter la question 1.3, on est amené à étudier la question :

Question 6.1. Soit $G$ un groupe linéaire. Soient $X$ et $Z$ deux $G$-variétés lisses géométriquement intègres. Soient $U \subset X$ un $G$-ouvert et $U \stackrel{f}{\rightarrow} Z$ un $G$-morphisme. Soient $B \subset \operatorname{Br}(U)$ un sousgroupe fini, $S \subset \Omega_{k}$ un sous-ensemble fini non vide et $W \subset X\left(\mathbf{A}_{k}\right)$ un ouvert. Sous quelles conditions peut-on montrer que, si $W^{\operatorname{Br}(U) \cap\left(B+f^{*} \operatorname{Br}_{G}(Z)\right)} \neq \emptyset$, alors il existe $z \in Z(k)$ de fibre $U_{z}$ tel que $\left(\left(G\left(k_{S}\right) \cdot W\right) \cap U_{z}\left(\mathbf{A}_{k}\right)\right)^{B} \neq \emptyset$ ?

Dans [CTH], Colliot-Thélène et Harari étudient la méthode de fibration au dessus de $\mathbb{A}^{1}$. On suit leur méthode et répond à la question 6.1 d'abord dans le cas où $Z$ est un tore quasi-trivial et $f$ s'étend à un morphisme de $X$ vers une $Z$-variété torique standard (Théorème 6.7). Ensuite, en utilisant ce résultat, on résoud cette question dans le cas où $Z$ est un tore (Théorème 6.9). En utilisant la descente $(\S 5)$, on établit le théorème 6.11 dans le cas plus général où $Z$ est un pseudo $G$-espace homogène. Ceci sera utilisé dans la section 7 pour traiter le cas d'un $G$-espace homogène $Z$ à stabilisateur géométrique connexe.

Rappelons la notion de sous-groupe de Brauer invariant (cf. Définition 3.1). 
6.1. L'approximation forte raffinée pour l'espace affine. Pour un ouvert $U$ d'un espace affine $\mathbb{A}^{n}$ satisfaisant $\operatorname{codim}\left(\mathbb{A}^{n} \backslash U, \mathbb{A}^{n}\right) \geq 2$, l'approximation forte hors d'une place $v_{0}$ a été établie par Fei Xu et l'auteur dans [CX, Prop. 3.6], et raffinée lorsque la place $v_{0}$ est archimédienne dans [CX1, Prop. 4.6 et Cor. 4.7 ], où l'on montre que $U(k) \cap T\left(k_{v_{0}}\right)^{+} \subset \mathbb{A}^{n}\left(k_{v_{0}}\right)$ est dense dans $U\left(\mathbf{A}_{k}^{v_{0}}\right)$ pour une variété torique $\left(T \hookrightarrow \mathbb{A}^{n}\right)$ comme (6.1) ci-dessous. On généralise maintenant ce résultat au cas où $v_{0}$ est une place quelconque.

Théorème 6.2. Soient $K$ une $k$-algèbre finie séparable de degré $n$ et

$$
\left(T \hookrightarrow \mathbb{A}^{n}\right):=\left(\operatorname{Res}_{K / k} \mathbb{G}_{m} \hookrightarrow \operatorname{Res}_{K / k} \mathbb{A}^{1}\right)
$$

la variété torique correspondante. Soit $U \subset \mathbb{A}^{n}$ un ouvert tel que $\operatorname{codim}\left(\mathbb{A}^{n} \backslash U, \mathbb{A}^{n}\right) \geq 2$. Soient $v_{0}$ une place et $D_{v_{0}} \subset T\left(k_{v_{0}}\right)$ un sous-groupe ouvert d'indice fini. Alors, pour tout ouvert $W \subset U\left(\mathbf{A}^{v_{0}}\right)$ non vide et tout ouvert $W_{0} \subset \mathbb{A}^{n}\left(k_{v_{0}}\right)$ non vide $D_{v_{0}}$-invariant, on a

$$
U(k) \cap\left(W_{0} \times W\right) \neq \emptyset .
$$

Démonstration. Étape (0). Par approximation faible pour le tore quasi-trivial $T$, il existe un $\alpha \in T(k) \cap W_{0}$. Ainsi on a $D_{v_{0}} \subset \alpha^{-1} \cdot W_{0}$. En remplaçant $U$ par $\alpha^{-1} U$ et $W$ par $\alpha^{-1} W$, on peut supposer que $W_{0}=D_{v_{0}}$. Si $v_{0}$ est complexe, on a $D_{v_{0}}=T\left(k_{v_{0}}\right)$ et l'énoncé découle de [CX, Prop. 3.6]. On suppose que $v_{0}$ n'est pas complexe.

Étape (1). Supposons que $v_{0} \in \infty_{k}$ et $T=\mathbb{G}_{m}^{n}$. Puisque $D_{v_{0}} \subset T\left(k_{v_{0}}\right)$ est ouvert et donc fermé, le sous-groupe $D_{v_{0}}$ contient la composante connexe de l'identité de $T\left(k_{v_{0}}\right)$. Ainsi l'énoncé est équivalent à [CX1, Prop. 4.6].

Étape (2). Supposons que $v_{0}<\infty_{k}, n=1$ et $T=\mathbb{G}_{m}$. On a $U=\mathbb{A}^{1}$. Par définition, Il existe un sous-ensemble fini $S \subset \Omega_{k} \backslash\left\{v_{0}\right\}$ contenant $\infty_{k}$, un élément $a_{v} \in k_{v}$ pour chaque $v \in S$ et $0<\epsilon<1$ tels que

$$
\prod_{v \in S}\left\{x \in k_{v}:\left|x-a_{v}\right|<\epsilon\right\} \times \prod_{v \notin S \cup\left\{v_{0}\right\}} \mathcal{O}_{v} \subset W .
$$

On fixe une uniformisante $\pi_{v_{0}}$ de $k_{v_{0}}$. Pour le sous-groupe d'indice fini $D_{v_{0}} \subset k_{v_{0}}^{\times}$, il existe un entier $N>0$ tel que

$$
\bigcup_{i \in \mathbb{Z}}\left(1+\pi_{v_{0}}^{N} \mathcal{O}_{v_{0}}\right) \cdot \pi_{v_{0}}^{i N} \subset D_{v_{0}} .
$$

Par approximation forte sur $\mathbb{A}^{1}$, il existe $\alpha, \beta \in k^{\times}$tels que

$$
\alpha \in k_{v_{0}} \times \prod_{v \in S}\left\{x \in k_{v}:\left|x-a_{v}\right|<\frac{1}{2} \epsilon\right\} \times \prod_{v \notin S \cup\left\{v_{0}\right\}} \mathcal{O}_{v}
$$

et

$$
\beta \in k_{v_{0}} \times \prod_{v \in S}\left\{x \in k_{v}:|x|<\frac{1}{2} \epsilon\right\} \times \prod_{v \notin S \cup\left\{v_{0}\right\}} \mathcal{O}_{v} .
$$

D'après la formule du produit, $l:=-v_{0}(\beta)>0$ et donc $\pi_{v_{0}}^{l} \beta \in \mathcal{O}_{v_{0}}^{\times}$. Alors il existe $m \in \mathbb{Z}$ assez grand tel que

$$
\left(\beta \pi_{v_{0}}^{l}\right)^{m} \in\left(1+\pi_{v_{0}}^{N} \mathcal{O}_{v_{0}}\right) \quad \text { et } \quad v_{0}\left(\alpha \beta^{-m}\right)>N
$$

Ainsi

$$
\alpha+\beta^{m N}=\pi_{v_{0}}^{-m l N}\left(\beta \pi_{v_{0}}^{l}\right)^{m N}\left(1+\alpha \beta^{-m N}\right) \in\left(1+\pi_{v_{0}}^{N} \mathcal{O}_{v_{0}}\right) \pi_{v_{0}}^{-m l N} \subset D_{v_{0}} .
$$

Ceci implique que $\alpha+\beta^{m N} \in D_{v_{0}} \times W$. 
Étape (3). Supposons que $v_{0}<\infty_{k}, T \cong \mathbb{G}_{m}^{n}$ et, sous cet isomorphisme, $D_{v_{0}} \cong \prod_{i=1}^{n} D_{i}$ avec $D_{i} \subset k_{v_{0}}^{\times}$un sous-groupe ouvert d'indice fini. On établi le résultat en utilisant projection $\mathbb{A}^{n} \rightarrow \mathbb{A}^{1}$ sur le premier facteur. L'argument donné pour $v_{0}$ réel dans [CX1, Prop. 4.6] vaut pour tout $v_{0}$ en remplaçant $\mathbb{R}$ par $D_{1}$.

Étape (4). En général, d'après (1) et (3), il reste à montrer qu'il existe des sous-groupes ouverts $\left\{D_{i}\right\}_{i=1}^{n}$ de $k_{v_{0}}^{\times}$d'indice fini et un isomorphisme

$$
\psi: \operatorname{Res}_{K / k} \mathbb{A}^{1} \rightarrow \mathbb{A}^{n} \quad \text { tels que } \prod_{i=1}^{n} D_{i} \subset \psi\left(D_{v_{0}}\right) .
$$

Si $v_{0}$ est réel, ceci est établi dans la démonstration de [CX1, Cor. 4.7]. On peut alors supposer que $v_{0}<\infty_{k}$ et $K=k(\theta)$ soit un corps.

Soit $\left\{w_{j}\right\}_{j}$ les places de $K$ au-dessus de $v_{0}$ et, pour tout $j$, soient $\pi_{j}$ une uniformisante de $K_{w_{j}}$ et $\mathcal{O}_{j}$ l'anneau des entiers de $K_{w_{j}}$ Pour $\pi_{v_{0}}$ une uniformisante de $k_{v_{0}}$ et pour chaque $j$, soit $e_{j}:=w_{j}\left(\pi_{v_{0}}\right) \leq n$. Puisque $D_{v_{0}}$ est un sous-groupe ouvert de $T\left(k_{v_{0}}\right) \cong \prod_{j} K_{w_{j}}^{\times}$, il existe un entier $N \in \mathbb{Z}_{>0}$ tel que

$$
\prod_{j}\left[\bigcup_{l \in \mathbb{Z}}\left(1+\pi_{j}^{N} \mathcal{O}_{j}\right) \pi_{j}^{l N}\right] \subset D_{v_{0}} .
$$

Après avoir remplacé $\theta$ par $\theta+\pi_{v_{0}}^{-c N}$ avec $c \gg 0$ suffisamment divisible, on peut supposer que

$$
w_{j}(\theta)=-e_{j} c N, \quad \theta \pi_{j}^{e_{j} c N} \in\left(1+\pi_{j}^{N} \mathcal{O}_{j}\right) \quad \text { et } \quad\left(\pi_{v_{0}} \pi_{j}^{-e_{j}}\right)^{c} \in\left(1+\pi_{j}^{N} \mathcal{O}_{j}\right) .
$$

Soit $M:=c n^{2} N$. Alors, pour tout $j$ et tous entiers $m, m^{\prime}$ avec $0 \leq m<m^{\prime} \leq n-1$, on a

$$
\left|m^{\prime}-m\right|\left|w_{j}(\theta)\right|<n e_{j} c N \leq M \quad \text { et donc } \quad 0<\left(w_{j}\left(\theta^{m}\right)-w_{j}\left(\theta^{m^{\prime}}\right)\right)<M .
$$

Donc, pour tous $\alpha, \alpha^{\prime} \in k_{v_{0}}^{\times}$satisfaisant $M \mid v_{0}(\alpha)$ et $M \mid v_{0}\left(\alpha^{\prime}\right)$, on a

$$
w_{j}\left(\alpha \theta^{m}\right) \neq w_{j}\left(\alpha^{\prime} \theta^{m^{\prime}}\right) \quad \text { et }\left\{\begin{array}{lll}
w_{j}\left(\alpha^{\prime}\right)>w_{j}(\alpha) & \text { si } & w_{j}\left(\alpha \theta^{m}\right)<w_{j}\left(\alpha^{\prime} \theta^{m^{\prime}}\right) \\
w_{j}(\alpha) \geq w_{j}\left(\alpha^{\prime}\right) & \text { si } & w_{j}\left(\alpha \theta^{m}\right)>w_{j}\left(\alpha^{\prime} \theta^{m^{\prime}}\right)
\end{array} .\right.
$$

Donc

$$
\left\{\begin{array}{lll}
w_{j}\left(\alpha^{\prime} \theta^{m^{\prime}}\right)-w_{j}\left(\alpha \theta^{m}\right)>M-\left(m^{\prime}-m\right)\left(-w_{j}(\theta)\right) & \text { si } & w_{j}\left(\alpha \theta^{m}\right)<w_{j}\left(\alpha^{\prime} \theta^{m^{\prime}}\right) \\
w_{j}\left(\alpha \theta^{m}\right)-w_{j}\left(\alpha^{\prime} \theta^{m^{\prime}}\right)>\left(m^{\prime}-m\right)\left(-w_{j}(\theta)\right) & \text { si } & w_{j}\left(\alpha \theta^{m}\right)>w_{j}\left(\alpha^{\prime} \theta^{m^{\prime}}\right)
\end{array} .\right.
$$

L'élément $\theta$ induit un isomorphisme $\psi: \operatorname{Res}_{K / k} \mathbb{A}^{1} \rightarrow \mathbb{A}^{n}$, qui est défini par

$$
\operatorname{Res}_{K / k} \mathbb{A}^{1}(A)=\sum_{i=0}^{n-1} A \theta^{i} \stackrel{\psi}{\rightarrow} \mathbb{A}^{n}(A)=A^{n}: \quad \sum_{i=0}^{n-1} a_{i} \theta^{i} \mapsto\left(a_{0}, \cdots, a_{n-1}\right)
$$

pour toute $k$-algèbre $A$. Soit

$$
D:=\bigcup_{l \in \mathbb{Z}}\left(1+\pi_{v_{0}}^{N} \mathcal{O}_{v_{0}}\right) \pi_{v_{0}}^{l M} \subset k_{v_{0}}^{\times}
$$

un sous-groupe ouvert d'indice fini. D'après (6.3), on a $D \subset \cup_{l \in \mathbb{Z}}\left(1+\pi_{j}^{N} \mathcal{O}_{j}\right) \pi_{j}^{l N}$. Pour tout $j$ et tout $\left(x_{0}, \cdots, x_{n-1}\right) \in D^{n}$, il existe un $0 \leq i_{j} \leq n-1$ tel que

$$
w_{j}\left(x_{i_{j}} \theta^{i_{j}}\right)=\min _{0 \leq i \leq n-1}\left\{w_{j}\left(x_{i} \theta^{i}\right)\right\}=w_{j}\left(\sum_{i=0}^{n-1} x_{i} \theta^{i}\right),
$$


où la deuxième égalité découle par (6.4). Pour $i \neq i_{j}$, d'après (6.5), on a

$$
w_{j}\left(x_{i} \theta^{i}\right)-w_{j}\left(x_{i_{j}} \theta^{i_{j}}\right) \geq \begin{cases}\left(i-i_{j}\right) e_{j} c N \geq N & \text { si } \quad i>i_{j} \\ M-\left(i_{j}-i\right) e_{j} c N \geq N & \text { si } \quad i<i_{j} .\end{cases}
$$

Alors

$$
\psi^{-1}\left(x_{0}, \cdots, x_{n-1}\right)=\sum_{i=0}^{n-1} x_{i} \theta^{i} \in x_{i_{j}} \theta^{i_{j}}\left(1+\pi_{j}^{N} \mathcal{O}_{j}\right) \subset \bigcup_{l \in \mathbb{Z}}\left(1+\pi_{j}^{N} \mathcal{O}_{j}\right) \pi_{j}^{l N} .
$$

D'après (6.2), on a $D^{n} \subset \psi\left(D_{v_{0}}\right)$.

On rappelle la définition des variétés toriques standards ([CX, Déf. 2.12])

Définition 6.3. Soit $K$ une $k$-algèbre finie séparable. La variété torique standard par rapport à $K / k$ est la sous-variété torique $\left(\operatorname{Res}_{K / k} \mathbb{G}_{m} \hookrightarrow Z\right)$ de $\left(\operatorname{Res}_{K / k} \mathbb{G}_{m} \hookrightarrow \operatorname{Res}_{K / k} \mathbb{A}^{1}\right)$ avec

$$
Z:=\operatorname{Res}_{K / k} \mathbb{A}^{1} \backslash\left[\left(\operatorname{Res}_{K / k} \mathbb{A}^{1} \backslash \operatorname{Res}_{K / k} \mathbb{G}_{m}\right)_{\text {sing }}\right] .
$$

Corollaire 6.4. Soit $(T \hookrightarrow Z)$ une variété torique standard. Soient $v_{0}$ une place de $k$ et $D_{v_{0}} \subset$ $T\left(k_{v_{0}}\right)$ un sous-groupe ouvert d'indice fini. Pour tout fermé $F \subset Z$ de codimension $\geq 2$ et tout ouvert non vide $W \subset(Z \backslash F)\left(\mathbf{A}_{k}\right)$, si $\left(D_{v_{0}} \cdot W\right) \cap(Z \backslash F)\left(\mathbf{A}_{k}\right)=W$, alors on a $T(k) \cap W \neq \emptyset$.

Démonstration. Puisque $\operatorname{codim}\left(\operatorname{Res}_{K / k} \mathbb{A}^{1} \backslash Z, \operatorname{Res}_{K / k} \mathbb{A}^{1}\right) \geq 2$, une application du théorème 6.2 donne le résultat.

6.2. Fibration sur une variété torique standard. On a besoin d'une généralisation du théorème de Tchebotarev "géométrique" (Ekedahl [E, Lem. 2.1]) :

Lemme 6.5. Soient $X, Y, Z$ des schémas intègres de type fini sur $\mathcal{O}_{k}$, et $Y \stackrel{p}{\rightarrow} X, X \stackrel{f}{\rightarrow} Z$ deux $\mathcal{O}_{k}$-morphismes. Supposons que $f$ et $f \circ p$ sont lisses à fibres géométriquement intègres et $p$ est fini étale galoisenne de groupe de Galois $\Gamma$. Soit $C$ une classe de conjugaison de $\Gamma$ et $c:=|C| /|\Gamma|$. Pour chaque place $v \in \Omega_{k}$ et chaque $z \in Z(k(v))$, soit $N_{C}(z)$ le nombre de $x \in X_{z}(k(v))$ tel que le Frobenius $F_{x}$ de $x$ soit dans $C$. Alors

$$
\frac{N_{C}(z)}{\left|X_{z}(k(v))\right|}-c=O\left(|k(v)|^{-1 / 2}\right)
$$

où la constante dans $O(-)$ ne dépend ni de $v$ ni de z.

Démonstration. Le résultat découle de la démonstration standard de [E, Lem. 2.1] avec la formule des traces de Lefschetz

$$
\sum_{x \in X_{z}(k(v))} \chi\left(F r_{x}\right)=\sum_{0 \neq i \neq 2 \operatorname{dim}\left(X_{z}\right)}(-1)^{i} \operatorname{Tr}\left(F r^{*}, H_{c}^{I}\left(X_{\bar{z}}\right), V_{\chi}\right),
$$

où $V_{\chi}$ est défini dans la démonstration de [E, Lem. 2.1]. Puisque $R^{i} f_{!} V_{\chi}$ est constructible et $H_{c}^{i}\left(X_{\bar{z}}, V_{\chi}\right)=\left(R^{i} f_{!} V_{\chi}\right)_{\bar{z}}$, les dimensions des $H_{c}^{i}\left(X_{\bar{z}}, V_{\chi}\right)$ sont bornées uniformément. De plus, la valeur absolue de la valeur propre de $F r^{*}$ en $H_{c}^{i}\left(X_{\bar{z}}, V_{\chi}\right)$ est inférieure ou égale à $|k(v)|^{\frac{i}{2}}$. Le reste de la démonstration est la même que celle de [E, Lem. 2.1]. 
Lemme 6.6. Soient $Z$ un ouvert de $\mathbb{A}^{n}$ satisfaisant $\operatorname{codim}\left(\mathbb{A}^{n} \backslash Z, \mathbb{A}^{n}\right) \geq 2$ et $V$ un ouvert de Z. Alors la suite exacte (3.3) induit un isomorphisme

$$
\operatorname{Br}_{a}(V) \stackrel{\partial}{\rightarrow} H^{1}\left(k, \operatorname{Div}_{Z_{\bar{k}} \backslash V_{\bar{k}}}\left(Z_{\bar{k}}\right) \otimes \mathbb{Q} / \mathbb{Z}\right) .
$$

Démonstration. On peut supposer que $Z \backslash V$ est lisse. Puisque $\operatorname{codim}\left(\mathbb{A}^{n} \backslash Z, Z\right) \geq 2$, on a $\operatorname{Br}_{a}(Z)=0$ et $H^{i}\left(Z_{\bar{k}}, \mathbb{Q} / \mathbb{Z}(1)\right)=0$ pour $i=1,2$. Puisque $V(k) \neq \emptyset$, le morphisme

$$
H^{3}(Z, \mathbb{Q} / \mathbb{Z}(1)) \rightarrow H^{3}(V, \mathbb{Q} / \mathbb{Z}(1)) \oplus H^{3}\left(Z_{\bar{k}}, \mathbb{Q} / \mathbb{Z}(1)\right)
$$

est donc injectif. Le résultat découle de la suite exacte (3.3).

Théorème 6.7. Soient $(T \hookrightarrow Z)$ une variété torique standard, $G$ un groupe linéaire connexe et $G \stackrel{\varphi}{\rightarrow} T$ un homomorphisme surjectif de noyau connexe. Soient $X$ une G-variété lisse, géométriquement intègre et $X \stackrel{f}{\rightarrow} Z$ un $G$-morphisme lisse surjectif à fibres géométriquement intègres. Notons $U:=X \times_{Z} T$. Soit $B \subset \operatorname{Br}_{G}(U)$ (cf. (3.1)) un sous-groupe fini. Soient $v_{0} \in \Omega_{k}$ une place et $G\left(k_{v_{0}}\right)^{0} \subset G\left(k_{v_{0}}\right)$ un sous-groupe d'indice fini. Alors, pour tout fermé $F \subset X$ de codimension $\geq 2$ et tout ouvert $W$ de $(X \backslash F)\left(\mathbf{A}_{k}\right)$ satisfaisant $W^{\operatorname{Br}(X) \cap\left(B+\left.f\right|_{U} ^{*} \operatorname{Br}_{1}(T)\right)} \neq \emptyset$, il existe $t \in T(k)$ de fibre $F_{t} \subset X_{t}$, tel que

$$
\operatorname{codim}\left(F_{t}, X_{t}\right) \geq 2 \quad \text { et } \quad\left(X_{t} \backslash F_{t}\right)\left(\mathbf{A}_{k}\right)^{B} \cap\left(G\left(k_{v_{0}}\right)^{0} \cdot W\right) \neq \emptyset .
$$

Démonstration. Puisque $f$ est lisse, il existe un fermé $F_{1} \subset Z$ tel que $f(X \backslash F)=Z \backslash F_{1}$.

Soient $\left\{C_{i}\right\}_{i \in I}$ les composantes connexes de $Z \backslash T$ et $D_{i}:=X \times{ }_{Z} C_{i}$ les composantes connexes de $X \backslash U$. Par hypothèse, les variétés $C_{i}$ et $D_{i}$ sont lisses intègres. D'après (3.3), on a une suite exacte :

$$
0 \rightarrow \operatorname{Br}(X) \cap\left(B+\left.f\right|_{U} ^{*} \operatorname{Br}_{1}(T)\right) \rightarrow\left(B+\left.f\right|_{U} ^{*} \operatorname{Br}_{1}(T)\right) \stackrel{\partial}{\rightarrow} \oplus_{i} H^{1}\left(D_{i}, \mathbb{Q} / \mathbb{Z}\right)
$$

Par le lemme 3.5, pour tout $i$, il existe un revêtement fini étale galoisien abélien $D_{i}^{\prime} \stackrel{\pi_{i}}{\rightarrow} D_{i}$ tel que $D_{i}^{\prime}$ soit une $G$-variété intègre, $\pi_{i}$ soit un $G$-morphisme et $\partial(b) \in H^{1}\left(D_{i}^{\prime} / D_{i}, \mathbb{Q} / \mathbb{Z}\right)$.

Soient $k_{i}$ la fermeture intégrale de $k$ dans $k\left(C_{i}\right)$ et $k_{i}^{\prime}$ la fermeture intégrale de $k$ dans $k\left(D_{i}^{\prime}\right)$. Par hypothèse, la fermeture intégrale $k$ dans $k\left(D_{i}\right)$ est $k_{i}$. D'après [CX1, Prop. 2.2], le morphisme $D_{i}^{\prime} \rightarrow C_{i} \times_{k_{i}} k_{i}^{\prime}$ est lisse à fibres géométriquement intègres.

Soit $B_{X}:=\operatorname{Br}(X) \cap\left(B+\left.f\right|_{U} ^{*} \operatorname{Br}_{1}(T)\right)$. D'après le lemme 3.2, $B_{X}$ est fini et $B_{X} \subset \operatorname{Br}_{G}(X)$. D'après le lemme 6.6 , on a un diagramme commutatif avec suite exacte :

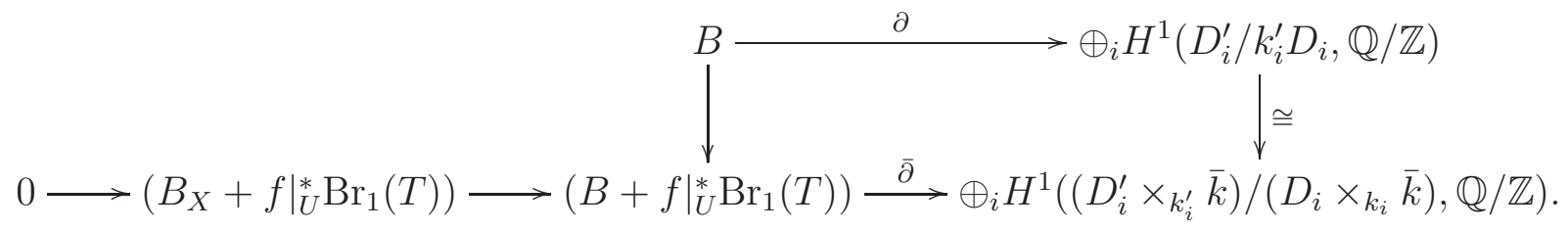

Soit $\operatorname{Br}_{G}(X) \stackrel{\lambda}{\rightarrow} \operatorname{Br}_{e}(G)$ l'homomorphisme de Sansuc (cf : Définition 3.8). Après avoir rétréci $G\left(k_{v_{0}}\right)^{0}$, on peut supposer que tout élément de $\lambda\left(B_{X}\right)$ s'annule sur $G\left(k_{v_{0}}\right)^{0}$. Par la proposition 3.9, pour tout $x \in X\left(\mathbf{A}_{k}\right)$, on a que tout élément de $B_{X}$ est constant sur $G\left(k_{v_{0}}\right)^{0} \cdot x$.

Soient $l:=\operatorname{dim}(Z), \Gamma_{i}:=\operatorname{Gal}\left(D_{i}^{\prime} / k_{i}^{\prime} D_{i}\right)$ et $W_{1}:=\left(G\left(k_{v_{0}}\right)^{0} \cdot W\right) \cap(X \backslash F)\left(\mathbf{A}_{k}\right)$.

Puisque $T$ est quasi-trivial, il existe des extensions de corps $L_{j} / k$ telles que $T \cong \operatorname{Res}_{\prod_{j} L_{j} / k} \mathbb{G}_{m}$. En fait, $\prod_{j} L_{j} \cong \prod_{i} k_{i}$, mais on ne l'utilise pas. Soit $\Omega_{0}$ l'ensemble des places $v \in \Omega_{k}$ totalement 
décomposées dans la fermeture galoisienne de $k_{i}^{\prime} / k$ pour tout $i$ et de $L_{j} / k$ pour tout $j$. Alors $\Omega_{0}$ est infini et pour tout $v \in \Omega_{0}$, on a $T_{k_{v}} \cong \mathbb{G}_{m, k_{v}}^{l}$. Par la définition 6.3 , on a

$$
Z_{k_{v}} \cong \operatorname{Spec} k_{v}\left[t_{1}, \cdots, t_{l}\right] \backslash \cup_{n \neq m} V\left(t_{n}, t_{m}\right) \quad \text { et } \quad T_{k_{v}} \cong \operatorname{Spec} k_{v}\left[t_{1}, t_{1}^{-1}, \cdots, t_{l}, t_{l}^{-1}\right]
$$

Soit $S$ un sous-ensemble fini de $\Omega_{k}$ tel que $v_{0} \cup \infty_{k} \subset S$. On agrandit $S$ de façon à avoir les propriétés suivantes :

(a) Le $k$-morphisme $f$ s'étend en un $\mathcal{O}_{S}$-morphisme lisse à fibres géométriquement intègres $\mathcal{X} \rightarrow \mathcal{Z}$ de $\mathcal{O}_{S^{-}}$-schémas lisses, tel que pour tout point fermé $z \in \mathcal{Z} \backslash \mathcal{F}_{1}$, la fibre $f^{-1}(z)$ possède un $k(z)$-point $x \notin \mathcal{F}$, où $\mathcal{F}$ est l'adhérence de $F$ dans $\mathcal{X}$ et $\mathcal{F}_{1}$ est l'adhérence de $F_{1}$ dans $\mathcal{Z}$. Ceci est possible par les estimées de Lang-Weil [Sko90, Thm. 1, étape 3]. Par le lemme de Hensel, pour tout $v \notin S$, l'application $(\mathcal{X} \backslash \mathcal{F})\left(\mathcal{O}_{v}\right) \rightarrow\left(\mathcal{Z} \backslash \mathcal{F}_{1}\right)\left(\mathcal{O}_{v}\right)$ est donc surjective.

(b) Les extensions $k_{i} / k$ et $k_{i}^{\prime} / k_{i}$ induisent des revêtements finis étales $\mathcal{O}_{k_{i}, S} / \mathcal{O}_{S}$ et $\mathcal{O}_{k_{i}^{\prime}, S} / \mathcal{O}_{k_{i}, S}$.

(c) Le sous-schéma $\mathcal{C}_{i}:=\overline{C_{i}} \subset \mathcal{Z}$ est lisse à fibres géométriquement intègres sur $\mathcal{O}_{k_{i}, S}$. Soient $\mathcal{D}_{i}:=\mathcal{C}_{i} \times_{\mathcal{Z}} \mathcal{X}, \mathcal{T}:=\mathcal{Z} \backslash \cup_{i} \mathcal{C}_{i}$ et $\mathcal{U}:=\mathcal{T} \times_{\mathcal{Z}} \mathcal{X}$

(d) Les éléments de $B_{X}$ appartiennent à $\operatorname{Br}(\mathcal{X})$ et les éléments de $B$ appartiennent à $\operatorname{Br}(\mathcal{U})$.

(e) Le revêtement $D_{i}^{\prime} \stackrel{\pi_{i}}{\rightarrow} D_{i}$ s'étend en un $\mathcal{O}_{S^{-}}$-revêtement fini étale galoisien abélien $\mathcal{D}_{i}^{\prime} \rightarrow \mathcal{D}_{i}$ tel que $\mathcal{D}_{i}^{\prime}$ soit un schéma sur $\mathcal{O}_{k_{i}^{\prime}, S}$, les résidus des éléments de $B$ soient dans $H^{1}\left(\mathcal{D}_{i}^{\prime} / \mathcal{D}_{i}, \mathbb{Q} / \mathbb{Z}\right)$ et $\mathcal{D}_{i}^{\prime} \rightarrow \mathcal{C}_{i} \times_{\mathcal{O}_{k_{i}, S}} \mathcal{O}_{k_{i}^{\prime}, S}$ soit lisse à fibres géométriquement intègres.

(f) Il existe un ouvert $W_{2} \subset W_{1} \subset(X \backslash F)\left(\mathbf{A}_{k}\right)$ tel que

$W_{2}^{B_{X}}=W_{2} \neq \emptyset, \quad W_{2}=\left(G\left(k_{v_{0}}\right)^{0} \cdot W_{2}\right) \cap(X \backslash F)\left(\mathbf{A}_{k}\right), \quad$ et $\quad W_{2}=W_{v_{0}} \times W_{S \backslash v_{0}} \times \prod_{v \notin S}(\mathcal{X} \backslash \mathcal{F})\left(\mathcal{O}_{v}\right)$

avec $W_{S \backslash v_{0}} \subset \prod_{v \in S \backslash\left\{v_{0}\right\}}(X \backslash F)\left(k_{v}\right)$ un ouvert et $W_{v_{0}} \subset(X \backslash F)\left(k_{v_{0}}\right)$ un ouvert.

(g) Pour tout $v \in \Omega_{0} \backslash\left(\Omega_{0} \cap S\right)$, on note $\mathcal{C}_{i, v}:=\mathcal{C}_{i} \times_{\mathcal{O}_{k_{i}, S}} \mathcal{O}_{v}, \mathcal{D}_{i, v}:=\mathcal{D}_{i} \times_{\mathcal{O}_{k_{i}, S}} \mathcal{O}_{v}$ et $\mathcal{D}_{i, v}^{\prime}:=\mathcal{D}_{i}^{\prime} \times_{\mathcal{O}_{k_{i}^{\prime}, S}} \mathcal{O}_{v}$. On a un diagramme commutatif de schémas intègres :

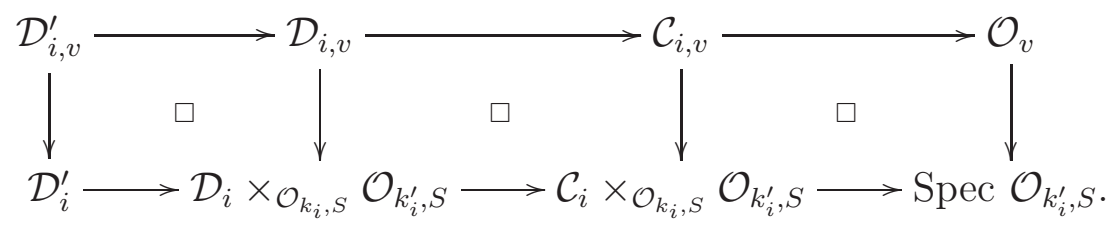

De plus, on a $\Gamma_{i} \cong \operatorname{Gal}\left(\mathcal{D}_{i}^{\prime} /\left(\mathcal{D}_{i} \times_{\mathcal{O}_{k_{i}, S}} \mathcal{O}_{k_{i}^{\prime}, S}\right)\right) \cong \operatorname{Gal}\left(\mathcal{D}_{i, v}^{\prime} / \mathcal{D}_{i, v}\right)$.

(h) Pour tout $v \in \Omega_{0} \backslash\left(\Omega_{0} \cap S\right)$, tout $\sigma \in \Gamma_{i}$ et tout $c \in \mathcal{C}_{i, v}(k(v))$ avec $c \notin \mathcal{F}_{1}$, la fibre $\left(\mathcal{D}_{i, v}\right)_{c}$ possède un $k(v)$-point $d$ avec $d \notin \mathcal{F}$ dont le Frobenius est $\sigma$. Ceci est possible en appliquant le lemme 6.5 à (6.8).

(i) Pour tout $v \in \Omega_{0} \backslash\left(\Omega_{0} \cap S\right)$, on note $(-)_{\mathcal{O}_{v}}:=(-) \times_{\mathcal{O}_{k, S}} \mathcal{O}_{v}$ et on a

$$
\mathcal{Z}_{\mathcal{O}_{v}} \cong \operatorname{Spec} \mathcal{O}_{v}\left[t_{1}, \cdots, t_{l}\right] \backslash \cup_{n \neq m} V\left(t_{n}, t_{m}\right), \quad \mathcal{T}_{\mathcal{O}_{v}} \cong \operatorname{Spec} \mathcal{O}_{v}\left[t_{1}, t_{1}^{-1}, \cdots, t_{l}, t_{l}^{-1}\right]
$$

et il existe une partition $\{1, \cdots, l\}=\coprod_{i} I_{i}$ telle que $\mathcal{C}_{i, \mathcal{O}_{v}}=\cup_{n \in I_{i}} V\left(t_{n}\right) \subset \mathcal{Z}$ et que $V\left(t_{n}\right) \cong \mathcal{C}_{i, v}$.

Pour chaque $i$, on choisit une place $v_{i} \in \Omega_{0} \backslash\left(\Omega_{0} \cap S\right)$ et un $n_{i} \in I_{i}$ tels que pour $i \neq j$, on ait $v_{i} \neq v_{j}$. Soient $\mathcal{C}_{i, n_{i}}:=V\left(t_{n_{i}}\right) \subset \mathcal{C}_{i, \mathcal{O}_{v_{i}}}, \mathcal{D}_{i, n_{i}}:=\mathcal{C}_{i, n_{i}} \times_{\mathcal{Z}} \mathcal{X}$ et

$$
E_{i}:=\left\{\left(t_{1}, \cdots, t_{l}\right) \in \mathcal{O}_{v_{i}}^{l}: t_{n_{i}} \in m_{v_{i}} \backslash m_{v_{i}}^{2} \text { et } t_{n} \in \mathcal{O}_{v_{i}}^{\times} \text {pour } n \neq n_{i}\right\}
$$


un ouvert de $\mathcal{Z}\left(\mathcal{O}_{v_{i}}\right)$. Alors $\mathcal{C}_{i, n_{i}} \cong \mathcal{C}_{i, v_{i}}, \mathcal{D}_{i, n_{i}} \cong \mathcal{D}_{i, v_{i}}$ et $\mathcal{D}_{i}^{\prime} \times_{\mathcal{D}} \mathcal{D}_{i, n_{i}} \cong \bigsqcup_{\left[k_{i}^{\prime}: k_{i}\right]} \mathcal{D}_{i, v_{i}}^{\prime}$. Donc le Frobenius en un point de $\mathcal{D}_{i, n_{i}}\left(k\left(v_{i}\right)\right)$ pour le revêtement $\mathcal{D}_{i}^{\prime} / \mathcal{D}_{i}$ est dans $\Gamma_{i}$.

Pour tout $b \in B$ et tout $P_{i} \in \mathcal{X}\left(\mathcal{O}_{v_{i}}\right)$ avec $f\left(P_{i}\right) \in E_{i}$, on a $\bar{P}_{i}:=P_{i}\left(k\left(v_{i}\right)\right) \in \mathcal{D}_{i, n_{i}}\left(k\left(v_{i}\right)\right)$ et alors la formule [Ha94, Cor. 2.4 .3 et p. 244-245] (voir [CTH, Formule (3.6)])

$$
b\left(P_{i}\right)=\partial_{i}(b)\left(F r_{\bar{P}_{i}}\right) \in \mathbb{Q} / \mathbb{Z},
$$

où $F r_{\bar{P}_{i}} \in \Gamma_{i} \subset \operatorname{Gal}\left(\mathcal{D}_{i}^{\prime} / \mathcal{D}_{i}\right)$ est le Frobenius en $\bar{P}_{i}$ pour le revêtement $\mathcal{D}_{i}^{\prime} / \mathcal{D}_{i}$ et

$$
\partial_{i}: \operatorname{Br}(\mathcal{U}) \stackrel{\partial}{\rightarrow} H^{1}\left(\mathcal{D}_{i}^{\prime} / \mathcal{D}_{i}, \mathbb{Q} / \mathbb{Z}\right) \rightarrow H^{1}\left(\mathcal{D}_{i, v_{i}}^{\prime} / \mathcal{D}_{i, v_{i}}, \mathbb{Q} / \mathbb{Z}\right)=\operatorname{Hom}\left(\Gamma_{i}, \mathbb{Q} / \mathbb{Z}\right) .
$$

Par fonctorialité, l'application $\bar{\partial}$ de (6.7) satisfait $\bar{\partial}=\oplus_{i} \partial_{i}$.

Notons $T\left(k_{v_{0}}\right)^{0}:=\varphi\left(G\left(k_{v_{0}}\right)^{0}\right)$. Puisque $H^{1}\left(k_{v_{0}}, \operatorname{Ker}(\varphi)\right)$ est fini ([PR, Thm. 6.14]), le sousgroupe $T\left(k_{v_{0}}\right)^{0} \subset T\left(k_{v_{0}}\right)$ est d'indice fini. D'après [Cod, Thm. 4.5], $f\left(W_{2}\right)$ est un ouvert de $\left(Z \backslash F_{1}\right)\left(\mathbf{A}_{k}\right)$. Pour tout $z \in\left(Z \backslash F_{1}\right)\left(k_{v_{0}}\right)$, l'ouvert $\left(X_{z} \backslash F_{z}\right)\left(k_{v_{0}}\right)$ est dense dans $X_{z}\left(k_{v_{0}}\right)$. Donc

$$
\left(T\left(k_{v_{0}}\right)^{0} \cdot f\left(W_{v_{0}}\right)\right) \cap\left(Z \backslash F_{1}\right)\left(k_{v_{0}}\right)=f\left(W_{v_{0}}\right) \text { et } \quad\left(T\left(k_{v_{0}}\right)^{0} \cdot f\left(W_{2}\right)\right) \cap\left(Z \backslash F_{1}\right)\left(\mathbf{A}_{k}\right)=f\left(W_{2}\right) \text {. }
$$

Par le corollaire 6.4, il existe $t \in T(k) \cap f\left(W_{2}\right)$ tel que $\left.t\right|_{v_{i}} \in E_{i}$ pour tout $i$ et que $\operatorname{codim}\left(F_{t}, X_{t}\right) \geq$ 2. Alors il existe $\left(P_{v}\right) \in W_{2}$ tel que $f\left(P_{v}\right)=t$.

Soit $t_{i} \in \mathcal{Z}\left(k\left(v_{i}\right)\right)$ la spécialisation de $t$, alors $t_{i} \in \mathcal{C}_{i, n_{i}}\left(k\left(v_{i}\right)\right)$ et $t_{i} \notin \mathcal{F}_{1}$. D'après (6.7), on a un diagramme avec suite exacte :

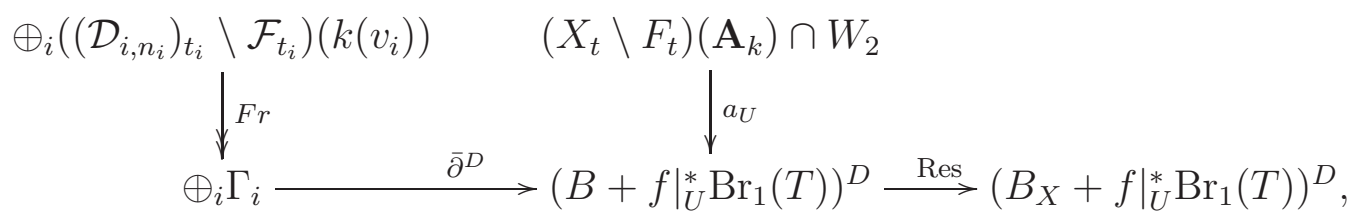

où $a_{U}$ est l'accouplement de Brauer-Manin, $(-)^{D}:=\operatorname{Hom}(-, \mathbb{Q} / \mathbb{Z})$ et, pour $u \in f^{-1}\left(t_{i}\right)$, l'élément $\operatorname{Fr}(u)$ est son Frobenius. Donc $F r$ est surjectif par (h). Puisque Res $\circ a_{U}=0$, il existe $\left\{\sigma_{i}\right\}_{i} \in \oplus_{i} \Gamma_{i}$ tel que $\bar{\partial}^{D}\left(\left\{\sigma_{i}\right\}\right)=a_{U}\left(\left\{P_{v}\right\}\right)$. Alors il existe $u_{i} \in\left(\left(\mathcal{D}_{i, n_{i}}\right)_{t_{i}} \backslash \mathcal{F}_{t_{i}}\right)\left(k\left(v_{i}\right)\right)$ tel que $\operatorname{Fr}\left(u_{i}\right)=\operatorname{Fr}\left(\bar{P}_{v_{i}}\right)-\sigma_{i}$. Par le lemme de Hensel, il existe un point $Q_{v_{i}} \in\left(\mathcal{X}_{t} \backslash \mathcal{F}_{t}\right)\left(\mathcal{O}_{v_{i}}\right)$ relevant $u_{i}$. Soit $Q_{v}:=P_{v}$ pour tout $v$ distinct de l'un des $v_{i}$. Par (6.9), $\left\{Q_{v}\right\}$ satisfait les conditions.

\subsection{Fibration sur un tore.}

Lemme 6.8. Soient $X$ et $Z$ deux variétés lisses géométriquement intègres, et $X \stackrel{f}{\rightarrow} Z$ un morphisme lisse surjectif à fibres géométriquement intègres. Soit $U \subset X$ un ouvert tel que $\left.f\right|_{U}$ soit surjectif. Soient $W \subset X\left(\mathbf{A}_{k}\right)$ un ouvert et $x \in W$. Alors il existe $u \in W \cap U\left(\mathbf{A}_{k}\right)$ tel que $f(u)=f(x)$.

Démonstration. Pour chaque $z \in Z$, la fibre $X_{z}$ est lisse intègre et l'ouvert $U_{z} \subset X_{z}$ est donc dense. Soit $\left\{z_{v}\right\}_{v}=f(x)$. Pour chaque $v$, l'ouvert $U_{z_{v}}\left(k_{v}\right)$ est dense en $X_{z_{v}}\left(k_{v}\right)$. Puisque $\left.f\right|_{U}$ est surjectif, le morphisme $\left.f\right|_{U}$ est lisse à fibres géométriquement intègres. Après avoir fixé un modèle intègre $\mathcal{U} \rightarrow \mathcal{Z}$ de $\left.f\right|_{U}$, on a que, pour presque toute place $v$, le morphisme $\mathcal{U}\left(\mathcal{O}_{v}\right) \rightarrow \mathcal{Z}\left(\mathcal{O}_{v}\right)$ est surjectif. Le résultat en découle.

Le théorème suivante, d'énoncé un peu technique, joue un rôle clé dans la démonstration du théorème 6.11 . 
Théorème 6.9. Soient $T, T_{0}$ deux tores avec $T_{0}$ quasi-trivial, $G$ un groupe linéaire connexe, $G \stackrel{\varphi}{\rightarrow} T_{0} \times T$ un homomorphisme surjectif de noyau connexe et $G_{0} \subset G$ un sous-groupe fermé connexe. Soient $X$ une $G$-variété lisse géométriquement intègre, $U \subset X$ un $G$-ouvert et $U \stackrel{f}{\rightarrow}$ $T_{0} \times T$ un $G$-morphisme. Soit $B \subset \operatorname{Br}_{G}(U)$ (cf. (3.1)) un sous-groupe fini. Supposons que:

(1) la composition $T_{0}^{*} \stackrel{p_{1}^{*}}{\rightarrow} T_{0}^{*} \times T^{*} \stackrel{f^{*}}{\rightarrow} \bar{k}[U]^{\times} / \bar{k}^{\times} \stackrel{\operatorname{div}_{X}}{\longrightarrow} \operatorname{Div}_{X_{\bar{k}} \backslash U_{\bar{k}}}\left(X_{\bar{k}}\right)$ est un isomorphisme;

(2) pour l'action de $G_{0}$ sur $X$, le morphisme $\bar{k}[X]^{\times} / \bar{k}^{\times} \rightarrow \bar{k}\left[G_{0}\right]^{\times} / \bar{k}^{\times}$défini par Sansuc ([S, (6.4.1)]) est injectif.

Alors, pour tout $v_{0} \in \Omega_{k}$, tout sous-groupe ouvert d'indice fini $G\left(k_{v_{0}}\right)^{0} \subset G\left(k_{v_{0}}\right)$ et tout ouvert $W \subset X\left(\mathbf{A}_{k}\right)$ satisfaisant $W^{\operatorname{Br}(X) \cap\left(B+f^{*} \mathrm{Br}_{1}\left(T_{0} \times T\right)\right)} \neq \emptyset$, il existe $t \in\left(T_{0} \times T\right)(k)$ de fibre $U_{t}$, tel que

$$
\left(G\left(k_{v_{0}}\right)^{0} \cdot G_{0}\left(k_{\infty}\right)^{+} \cdot W\right) \cap U_{t}\left(\mathbf{A}_{k}\right)^{B} \neq \emptyset .
$$

Démonstration. Par la proposition 2.3, après avoir remplacé $f \operatorname{par} \widetilde{\phi} \circ f$ et $\varphi$ par $\widetilde{\phi} \circ \varphi$ avec $\widetilde{\phi}$ un automorphisme de $T_{0} \times T$, on peut supposer que :

(i) il existe une variété torique $\left(T_{0} \hookrightarrow \mathbb{A}^{l}\right)$ satisfaisant $(2.1)$;

(ii) le morphisme $f$ s'étend à un $G$-morphisme $X \stackrel{f_{X}}{\longrightarrow} Z$ où $Z:=\mathbb{A}^{l} \times T \supset T_{0} \times T$;

(iii) on a $f_{X}(U) \subset T_{0} \times T$ et un isomorphisme $\operatorname{Div}_{Z_{\bar{k}} \backslash\left(T_{0} \times T\right)_{\bar{k}}}\left(Z_{\bar{k}}\right) \stackrel{f_{X}^{*}}{\longrightarrow} \operatorname{Div}_{X_{\bar{k}} \backslash U_{\bar{k}}}\left(X_{\bar{k}}\right)$.

Soit $Z_{0}:=\mathbb{A}^{l} \backslash\left[\left(\mathbb{A}^{l} \backslash T_{0}\right)_{\text {sing }}\right]$. Alors $\left(T_{0} \hookrightarrow Z_{0}\right)$ est une variété torique standard et

$$
Z_{1}:=Z_{0} \times T \cong Z \backslash\left[\left(Z \backslash T_{0} \times T\right)_{\text {sing }}\right]
$$

D'après la proposition 2.2, il existe un $G$-ouvert $X_{1} \subset X$ tel que $f\left(X_{1}\right) \subset Z_{1}, X_{1} \cap f_{X}^{-1}(T)=U$, $\operatorname{codim}\left(X \backslash X_{1}, X\right) \geq 2, \operatorname{Br}(X) \cong \operatorname{Br}\left(X_{1}\right)$ et que le morphisme $X_{1} \stackrel{\left.f_{X}\right|_{X_{1}}}{\longrightarrow} Z_{1}$ soit lisse surjectif à fibres géométriquement intègres.

Notons $\phi: X \stackrel{f_{X}}{\longrightarrow} \mathbb{A}^{l} \times T \stackrel{p_{2}}{\rightarrow} T$. Pour chaque $t \in T(k)$, notons $X_{1, t}:=\phi^{-1}(t) \cap X_{1}$, $U_{t}:=U \cap X_{1, t}, X_{1, t} \stackrel{i_{t}}{\rightarrow} X_{1}$ et $X_{1, t} \stackrel{f_{t}}{\rightarrow} Z_{0}$. On a le diagramme :

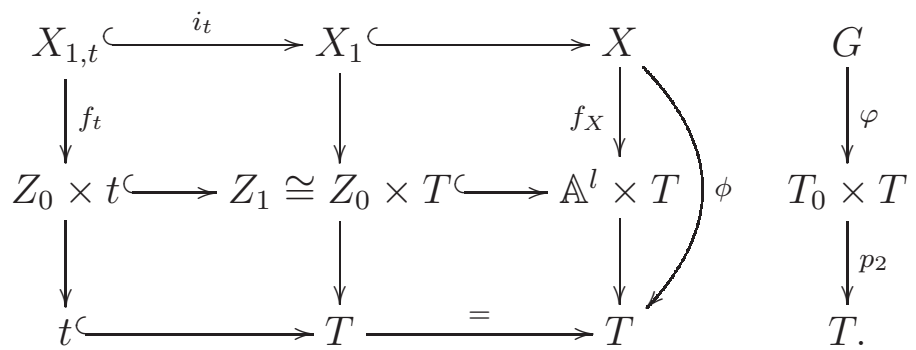

On a les propriétés ci-dessous :

(a) le morphisme $f_{t}$ satisfait les hypothèses géométriques du théorème 6.7 par rapport à $\operatorname{Ker}\left(G \stackrel{p_{2} \circ \varphi}{\longrightarrow} T\right) \rightarrow T_{0}$

(b) l'homomorphisme $G_{0} \stackrel{p_{2} \circ \psi}{\longrightarrow} T$ est surjectif et donc $\left(p_{2} \circ \psi\right)\left(G_{0}\left(k_{\infty}\right)^{+}\right)=T\left(k_{\infty}\right)^{+}$;

(c) Soient $B_{1}:=B+f^{*} \operatorname{Br}_{1}\left(T_{0} \times T\right)$ et $B_{2} \subset\left(\operatorname{Br}(X) \cap B_{1}\right)$ un sous-groupe fini tels que le morphisme $B_{2} \rightarrow \frac{\operatorname{Br}(X) \cap B_{1}}{\operatorname{Br}(X) \cap f^{*} \operatorname{Br}_{1}\left(T_{0} \times T\right)}$ soit surjectif, alors $i_{t}^{*} B \cap \operatorname{Br}\left(X_{1, t}\right) \subset i_{t}^{*} B_{2}$.

L'énoncé (a) est claire.

Pour (b), d'après [CX1, Prop. 2.2], $\phi$ est lisse surjectif à fibres géométriquement intègres. Donc $\bar{k}[T]^{\times} / \bar{k}^{\times} \stackrel{\phi^{*}}{\longrightarrow} \bar{k}[X]^{\times} / \bar{k}^{\times}$est injectif. Notons $\bar{k}[X]^{\times} / \bar{k}^{\times} \stackrel{\theta_{X}}{\longrightarrow} \bar{k}\left[G_{0}\right]^{\times} / \bar{k}^{\times}$et $\bar{k}[T]^{\times} / \bar{k}^{\times} \stackrel{\theta_{T}}{\longrightarrow}$ 
$\bar{k}\left[G_{0}\right]^{\times} / \bar{k}^{\times}$les morphismes définis par Sansuc ([S, (6.4.1)]). Ainsi $\theta_{T}=\theta_{X} \circ \phi^{*}$ est injectif. Par l'argument de Sansuc ([S, P. 39]), $\theta_{T}=\left(\left.\left(p_{2} \circ \psi\right)\right|_{G_{0}}\right)^{*}$. Alors $G_{0} \stackrel{p_{2} \circ \psi}{\longrightarrow} T$ est surjectif.

Pour (c), d'après le lemme 3.2, on a

$$
\operatorname{Br}\left(X_{1}\right) \cap f^{*} \operatorname{Br}_{1}\left(T_{0} \times T\right) \cong f_{X}^{*} \operatorname{Br}_{1}\left(Z_{1}\right) \cong \phi^{*} \operatorname{Br}_{1}(T) \text { et } \quad B_{1} \cap \operatorname{Br}\left(X_{1}\right)=B_{2}+\phi^{*} \operatorname{Br}_{1}(T) \text {. }
$$

Par le corollaire 3.14, on a

$$
i_{t}^{*} B_{2}=i_{t}^{*}\left(B_{2}+\phi^{*} \operatorname{Br}_{1}(T)\right)=i_{t}^{*}\left(B_{1} \cap \operatorname{Br}\left(X_{1}\right)\right)=i_{t}^{*} B_{1} \cap \operatorname{Br}\left(X_{1, t}\right) \supset i_{t}^{*} B \cap \operatorname{Br}\left(X_{1, t}\right) .
$$

Ceci donne (c).

On considère l'ouvert $W$ de l'énoncé. Après avoir rétréci $W$, on peut supposer que tout élément de $B_{2}$ s'annule sur $W$.

On note $W_{1}:=W \cap X_{1}\left(\mathbf{A}_{k}\right)$. Soit $x \in W^{\phi^{*} \operatorname{Br}_{1}(T)}$. En appliquant le lemme 6.8 au triple $\left(X_{1} \subset X, X \stackrel{\phi}{\rightarrow} T, W\right)$, on voit qu'il existe $x_{1} \in W_{1}$, tel que $\phi\left(x_{1}\right)=\phi(x)$. Donc $W_{1}^{\phi^{*} \operatorname{Br}_{1}(T)} \neq \emptyset$ et $\phi\left(W_{1}\right)^{\operatorname{Br}_{1}(T)} \neq \emptyset$.

Soit $W_{2}:=G_{0}\left(k_{\infty}\right)^{+} \cdot W_{1}$. D'après (b), on a $\phi\left(W_{2}\right)=T\left(k_{\infty}\right)^{+} \cdot \phi\left(W_{1}\right)$. Puisque $T$ satisfait l'approximation forte par rapport à $\operatorname{Br}_{1}(T)$ hors de $\infty_{k}$ (Harari [Ha08, Thm. 2]), il existe $t \in T(k) \cap \phi\left(W_{2}\right)$. Donc $X_{1, t}\left(\mathbf{A}_{k}\right) \cap W_{2} \neq \emptyset$. D'après (c), $\left(X_{1, t}\left(\mathbf{A}_{k}\right) \cap W_{2}\right)^{i *} B \cap \operatorname{Br}\left(X_{1, t}\right) \neq \emptyset$.

Soit $W_{3}:=G\left(k_{v_{0}}\right)^{0} \cdot W_{2} \supset W_{2}$. D'après le théorème 6.7 , il existe $u \in W_{3} \cap U_{t}\left(\mathbf{A}_{k}\right)^{i_{t}^{*} B}$ tel que $f_{t}(u) \in T_{0}(k)$.

Corollaire 6.10. Avec les hypothèses et notations du théorème 6.9, soit $F \subset X$ un sous-schéma fermé $G_{0}$-invariant de codimension $\geq 2$. Alors, pour tout $v_{0} \in \Omega_{k}$ et tout $\tilde{W} \subset(X \backslash F)\left(\mathbf{A}_{k}\right)$ satisfaisant $\tilde{W}^{\mathrm{Br}(X) \cap\left(B+f^{*} \mathrm{Br}_{1}\left(T_{0} \times T\right)\right)} \neq \emptyset$, il existe un $t \in\left(T_{0} \times T\right)(k)$ tel que

$$
\operatorname{codim}\left(F \cap U_{t}, U_{t}\right) \geq 2 \text { et }\left(G\left(k_{v_{0}}\right)^{0} \cdot G_{0}\left(k_{\infty}\right)^{+} \cdot \tilde{W}\right) \cap\left(U_{t} \backslash\left(F \cap U_{t}\right)\right)\left(\mathbf{A}_{k}\right)^{B} \neq \emptyset .
$$

Démonstration. Avec les constructions et notations de la démonstration du théorème 6.9, soit $\tilde{W}_{1}:=\tilde{W} \cap\left(X_{1} \backslash F\right)\left(\mathbf{A}_{k}\right), \quad \tilde{W}_{2}:=G_{0}\left(k_{\infty}\right)^{+} \cdot \tilde{W}_{1} \quad$ et $\quad \tilde{W}_{3}:=\left(G\left(k_{v_{0}}\right)^{0} \cdot \tilde{W}_{2}\right) \cap\left(X_{1} \backslash F\right)\left(\mathbf{A}_{k}\right)$.

Le résultat découle du même argument que dans la démonstration du théorème 6.9, en remplaçant $W_{1}, W_{2}, W_{3}$ par $\tilde{W}_{1}, \tilde{W}_{2}, \tilde{W}_{3}$.

6.4. Fibration sur un pseudo espace homogène. Soit $G$ un groupe linéaire connexe. Rappelons la notion de pseudo $G$-espace homogène (cf. Définition 3.15). Soit $Z$ un pseudo $G$-espace homogène, on peut définir son quotient torique maximal $Z \stackrel{\pi}{\rightarrow} Z^{\text {tor }}$ et le stabilisateur de $G$ sur $Z^{\text {tor }}$ (cf. Définition 3.23).

Théorème 6.11. Soient $G$ un groupe linéaire connexe, $Z$ un pseudo $G$-espace homogène, $Z \stackrel{\pi}{\rightarrow}$ $Z^{\text {tor }}$ le quotient torique maximal et $G_{0}$ le stabilisateur de $G$ sur $Z^{\text {tor }}$. Soit $X$ une $G$-variété lisse géométriquement intègre telle que $\bar{k}[X]^{\times} / \bar{k}^{\times}=0$. Soient $U \subset X$ un $G$-ouvert et $U \stackrel{f}{\rightarrow} Z$ un $G$-morphisme. Soient $A \subset \operatorname{Br}(X), B \subset \operatorname{Br}_{G}(U)$ (cf. (3.1)) deux sous-groupes finis. Pour tout ouvert $W \subset X\left(\mathbf{A}_{k}\right)$ satisfaisant $W^{\mathrm{Br}(X) \cap\left(A+B+f^{*} \operatorname{Br}_{G}(Z)\right)} \neq \emptyset$, on a :

(1) pour toute place $v_{0} \in \Omega_{k}$ et tout sous-groupe ouvert d'indice fini $G\left(k_{v_{0}}\right)^{0} \subset G\left(k_{v_{0}}\right)$, il existe un $t \in Z^{\text {tor }}(k)$ de fibre $U_{t} \stackrel{f_{t}}{\rightarrow} Z_{t}$, tel que

$$
\left(G\left(k_{v_{0}}\right)^{0} \cdot W\right) \cap U_{t}\left(\mathbf{A}_{k}\right)^{A+B+f_{t}^{*} \operatorname{Br}_{G_{0}}\left(Z_{t}\right)} \neq \emptyset ;
$$


(2) s'il existe un sous-ensemble fini non vide $S \subset \Omega_{k}$ tel que, pour tout sous-groupe ouvert d'indice fini $G_{0}\left(k_{S}\right)^{0} \subset G_{0}\left(k_{S}\right)$ et tout $t \in Z^{\text {tor }}(k)$ de fibre $Z_{t}$, l'adhérence $\overline{G_{0}\left(k_{S}\right)^{0} \cdot Z_{t}(k)}$ contient $Z_{t}\left(\mathbf{A}_{k}\right)^{\operatorname{Br}_{G_{0}}\left(Z_{t}\right)}$, alors, pour tout sous-groupe ouvert d'indice fini $G\left(k_{S}\right)^{0} \subset G\left(k_{S}\right)$, il existe un $z \in Z(k)$ de fibre $U_{z}$ tel que $\left(G\left(k_{S}\right)^{0} \cdot W\right) \cap U_{z}\left(\mathbf{A}_{k}\right)^{A+B} \neq \emptyset$.

Démonstration. On considère (1).

Soient $T_{0}$ un tore tel que $T_{0}^{*} \cong \operatorname{Div}_{X_{\bar{k}} \backslash U_{\bar{k}}}\left(X_{\bar{k}}\right)$ et $Y_{0} \rightarrow X$ le $T_{0}$-torseur induit par l'homomorphisme $\Psi$ de la suite exacte (2.2).

D'après la proposition 5.7, il existe un tore quasi-trivial $T_{1}$ et un $T_{1}$-torseur $Z_{1} \stackrel{p_{1, Z}}{\longrightarrow} Z$ tels que $\operatorname{Pic}\left(Z_{1, \bar{k}}\right)=0$ et $H^{3}\left(k, \bar{k}\left[Z_{1}\right]^{\times} / \bar{k}^{\times}\right)=0$. Par le théorème 2.7 et le corollaire 2.9 , il existe un groupe linéaire connexe $H$ muni d'un homomorphisme surjectif $H \rightarrow G$ de noyau central $T_{1}$ tel que $Z_{1}$ soit une $H$-variété et que $p_{1, Z}$ soit un $H$-morphisme. De plus, $Z_{1}(k) \neq \emptyset$ et, d'après la proposition 3.17, $Z_{1}$ est un pseudo $H$-espace homogène.

Soient $T_{2}:=T_{0} \times T_{1}$ et $V_{1}:=U \times_{Z} Z_{1}$ un $T_{1}$-torseur sur $U$. Puisque $H^{1}\left(X, T_{1}\right) \rightarrow H^{1}\left(U, T_{1}\right)$ est surjectif, il existe un $T_{1}$-torseur $Y_{1} \rightarrow Y$ tel que $\left[Y_{1}\right]_{U}=\left[V_{1}\right]$. L'isomorphisme canonique $H^{1}\left(X, T_{0}\right) \oplus H^{1}\left(X, T_{1}\right) \stackrel{\theta}{\rightarrow} H^{1}\left(X, T_{2}\right)$ donne un $T_{2}$-torseur $Y \rightarrow X$ tel que $[Y]=\theta\left(\left[Y_{0}\right],\left[Y_{1}\right]\right)$. Maintenant on obtient des $T_{1}$-torseurs $Z_{1} \rightarrow Z, V_{1} \rightarrow U$ et des $T_{2}$-torseurs $Y \rightarrow X, V \rightarrow U$ tels que $f^{*}\left[Z_{1}\right]=\left[V_{1}\right],\left.[Y]\right|_{U}=[V]$ et $[V]=\left[T_{0} \times V_{1}\right]$.

Par le théorème 2.7, le corollaire 2.8 et le corollaire 2.9, il existe un homomorphisme surjectif $T_{0} \times H \stackrel{\psi}{\rightarrow} G$ de noyau central $T_{2}$ et un diagramme commutatif de $T_{0} \times H$-variétés et de $T_{0} \times H$-morphismes :

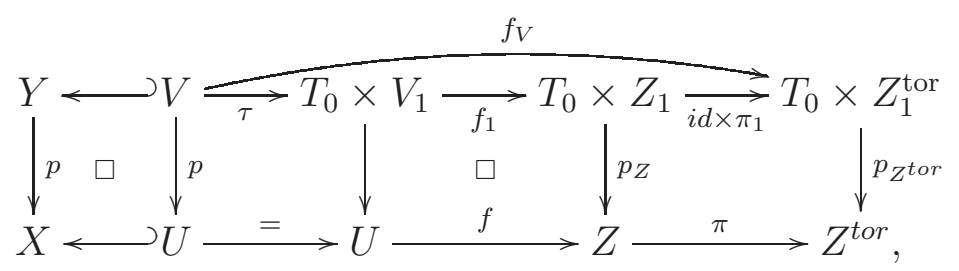

où $\tau$ est une trivialisation, $Z_{1} \stackrel{\pi_{1}}{\rightarrow} Z_{1}^{\text {tor }}$ est le quotient torique maximal, $f_{V}:=\left(i d \times \pi_{1}\right) \circ f_{1} \circ \tau$ est la composition et $p_{Z}:=p_{1, Z} \circ p_{2}$.

On a des propriétés :

(a) on peut supposer que la composition

$$
T_{0}^{*} \stackrel{p_{1}^{*}}{\rightarrow} \bar{k}\left[T_{0} \times V_{1}\right]^{\times} / \bar{k}^{\times} \stackrel{\tau^{*}}{\rightarrow} \bar{k}[V]^{\times} / \bar{k}^{\times} \stackrel{\operatorname{div}}{\longrightarrow} \operatorname{Div}_{Y_{\bar{k}} \backslash V_{\bar{k}}}\left(Y_{\bar{k}}\right)
$$

est un isomorphisme.

(b) le stabilisateur $H_{0}$ de $H$ sur $Z_{1}^{\text {tor }}$ est connexe et donc les morphismes $f_{V}, \pi, \pi_{1}$ et $\pi \circ f$ sont lisses à fibres géométriquement intègres ([CX1, Prop. 2.2]).

(c) on a

$$
X\left(\mathbf{A}_{k}\right)^{\operatorname{Br}(X) \cap\left(B+f^{*} \operatorname{Br}_{G}(Z)\right)}=p\left(Y\left(\mathbf{A}_{k}\right)^{\operatorname{Br}(Y) \cap\left[p^{*} B+\left(f_{1} \circ \tau\right)^{*} \operatorname{Br}_{T_{0} \times H}\left(T_{0} \times Z_{1}\right)\right]}\right) .
$$

(d) il existe un sous groupe fini $B_{1} \subset \mathrm{Br}_{T_{0} \times H}(V)$ tel que

$$
B_{1}+f_{V}^{*} \operatorname{Br}_{1}\left(T_{0} \times Z_{1}^{\text {tor }}\right)=\left(f_{1} \circ \tau\right)^{*} \operatorname{Br}_{T_{0} \times H}\left(T_{0} \times Z_{1}\right) \subset \operatorname{Br}(V) .
$$


(e) pour tout $\left(t_{0}, t_{1}\right) \in\left(T_{0} \times Z_{1}^{\text {tor }}\right)(k)$, notons $V_{\left(t_{0}, t_{1}\right)} \stackrel{\left.f_{1}\right|_{\left(t_{0}, t_{1}\right)}}{\longrightarrow} t_{0} \times Z_{1, t_{1}} \rightarrow\left(t_{0}, t_{1}\right)$ la fibre de $V \stackrel{f_{1} \circ \tau}{\longrightarrow} T_{0} \times Z_{1} \rightarrow T_{0} \times Z_{1}^{\text {tor }}$ et on a que la restriction $\operatorname{Br}_{T_{0} \times H}\left(T_{0} \times Z_{1}\right) \rightarrow \mathrm{Br}_{H_{0}}\left(Z_{1, t_{1}}\right)$ est surjective.

L'énoncé (a) résulte de la proposition 2.5. La proposition 3.22 et le lemme 3.21 donne (b).

Pour (c), puisque $\operatorname{Pic}\left(T_{2}\right)=0$, par le corollaire 3.11 et la suite exacte de Sansuc [S, Prop. 6.10], on a deux diagrammes commutatifs de suites exactes

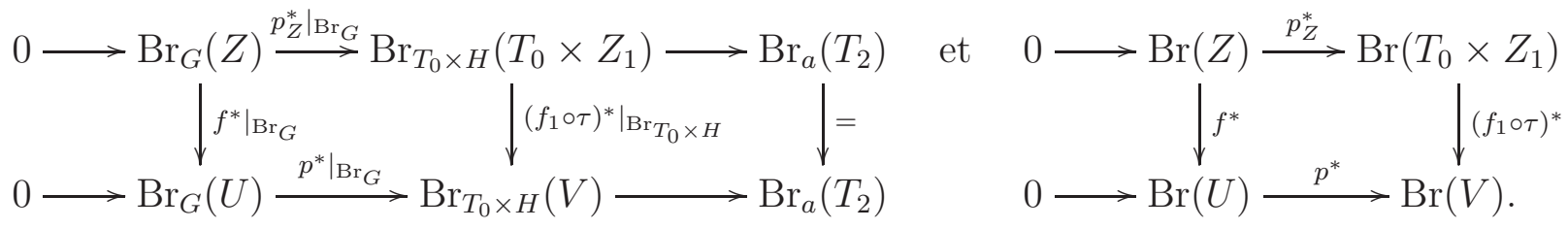

et $\left(p^{*}\right)^{-1} \operatorname{Br}_{T_{0} \times H}(V)=\operatorname{Br}_{G}(U)$. Donc $\left(p^{*}\right)^{-1}\left(\left(f_{1} \circ \tau\right)^{*} \operatorname{Br}_{T_{0} \times H}\left(T_{0} \times Z_{1}\right)\right)=f^{*} \operatorname{Br}_{G}(Z)$. Une application du corollaire 5.3 au torseur $Y \stackrel{p}{\rightarrow} X$ et aux sous-groupes :

$$
\left(B+f^{*} \operatorname{Br}_{G}(Z)\right) \subset \operatorname{Br}(U) \quad \text { et } \quad\left(f_{1} \circ \tau\right)^{*} \operatorname{Br}_{T_{0} \times H}\left(T_{0} \times Z_{1}\right) \subset \operatorname{Br}_{T_{0} \times H}(V)
$$

donne (c).

Pour (d), par la construction, on a $\bar{k}\left[Z_{1}^{\text {tor }}\right]^{\times} \cong \bar{k}\left[Z_{1}\right]^{\times}, \operatorname{Pic}\left(Z_{1, \bar{k}}\right)=0$ et $Z_{1}(k) \neq \emptyset$. Par la suite spectrale de Hochschild-Serre et [S, Lem. 6.6], on a $\operatorname{Br}_{1}\left(T_{0} \times Z_{1}^{\text {tor }}\right) \cong \operatorname{Br}_{1}\left(T_{0} \times Z_{1}\right)$. L'énoncé (d) découle de la proposition 3.18.

Pour (e), puisque $H^{3}\left(k, Z_{1}^{\text {tor,*}}\right)=0$, d'après le lemme 5.5, le morphisme $\operatorname{Br}_{a}(H) \rightarrow \operatorname{Br}_{a}\left(H_{0}\right)$ est surjectif. La proposition 3.13 donne (e).

On considère l'ouvert $W$ de l'énoncé. Après avoir rétréci $W$, on peut supposer que tout élément de $A$ s'annule sur $W$. D'après (c) et $(\mathrm{d})$, on a $\left(p^{-1}(W)\right)^{\operatorname{Br}(Y) \cap\left(p^{*} B+B_{1}+f_{V}^{*} \operatorname{Br}_{1}\left(T_{0} \times Z_{1}^{\text {tor }}\right)\right)} \neq \emptyset$.

Par la suite exacte de Sansuc [S, Prop. 6.10], le morphisme canonique $\bar{k}[Y]^{\times} / \bar{k}^{\times} \rightarrow \bar{k}\left[T_{2}\right]^{\times} / \bar{k}^{\times}$ est injectif. Puisque $p^{*} B+B_{1} \subset \mathrm{Br}_{T_{0} \times H}(V)$ est fini, une application du théorème 6.9 au triple

$$
\left(T_{0} \times H \rightarrow T_{0} \times Z_{1}^{\text {tor }}, V \subset Y, V \stackrel{f_{V}}{\longrightarrow} T_{0} \times Z_{1}^{\text {tor }}\right)
$$

montre qu'il existe

$v \in\left[\left(T_{0} \times H\right)\left(k_{v_{0}}\right)^{0} \cdot T_{2}\left(k_{\infty}\right)^{+} \cdot p^{-1}(W)\right] \cap V\left(\mathbf{A}_{k}\right)^{p^{*} B+B_{1}}$ tel que $\left(t_{0}, t_{1}\right):=f_{V}(v) \in\left(T_{0} \times Z_{1}^{\text {tor }}\right)(k)$, où $\left(T_{0} \times H\right)\left(k_{v_{0}}\right)^{0}:=\psi^{-1}\left(G\left(k_{v_{0}}\right)^{0}\right)$.

D'après (d) et (e) on a $\left.\left.v \in V_{\left(t_{0}, t_{1}\right)}\left(\mathbf{A}_{k}\right)^{p^{*} B+f_{1}}\right|_{\left(t_{0}, t_{1}\right)} ^{*} \operatorname{Br}_{H_{0}}\left(Z_{1, t_{1}}\right)\right)$. Donc $t:=\pi_{Z^{\text {tor }}}\left(\left(t_{0}, t_{1}\right)\right) \in Z^{\text {tor }}(k)$ et $u:=p(v) \in\left(G\left(k_{v_{0}}\right)^{0} \cdot W\right) \cap U_{t}\left(\mathbf{A}_{k}\right)^{B+f^{*} \operatorname{Br}_{G_{0}}\left(Z_{t}\right)}$. Ce qui donne (1).

On considère (2).

Fixons $v_{0} \in S$. On a le plongement canonique de groupes $G\left(k_{v_{0}}\right) \subset G\left(k_{S}\right)$. Puisque $G\left(k_{S}\right)^{0} \subset$ $G\left(k_{S}\right)$ est ouvert d'indice fini, les sous-groupes

$$
G\left(k_{v_{0}}\right)^{0}:=G\left(k_{S}\right)^{0} \cap G\left(k_{v_{0}}\right) \subset G\left(k_{v_{0}}\right) \text { et } G_{0}\left(k_{S}\right)^{0}:=G\left(k_{S}\right)^{0} \cap G_{0}\left(k_{S}\right) \subset G_{0}\left(k_{S}\right)
$$

sont ouverts d'indice fini. Pour tout $t \in Z^{\text {tor }}(k)$, l'ensemble $W_{t}:=\left(G\left(k_{v_{0}}\right)^{0} \cdot W\right) \cap U_{t}\left(\mathbf{A}_{k}\right)^{A+B}$ est ouvert dans $U_{t}\left(\mathbf{A}_{k}\right)$. D'après (1), il existe $t \in Z^{\text {tor }}(k)$ tel que $W_{t}^{f^{*} \operatorname{Br}_{G_{0}}\left(Z_{t}\right)} \neq \emptyset$ et donc $f_{t}\left(W_{t}\right)^{\operatorname{Br}_{G_{0}}\left(Z_{t}\right)} \neq \emptyset$. D'après [Cod, Thm. 4.5], $f_{t}\left(W_{t}\right) \subset Z_{t}\left(\mathbf{A}_{k}\right)$ est ouvert. Par hypothèse, il existe $z \in Z_{t}(k) \cap f_{t}\left(G_{0}\left(k_{S}\right)^{0} \cdot W_{t}\right)$ et ceci établit (2). 
Remarque 6.12. On peut établir le théorème 4.6 par la méthode de la démonstration du théorème 6.11. Mais l'argument dans $§ 4$ est plus simple.

Corollaire 6.13. Avec les hypothèses et notations du théorème 6.11 , soit $F \subset X$ sous-schéma fermé de codimension $\geq 2$. Alors, pour tout $v_{0} \in \Omega_{k}$, tout sous-groupe ouvert d'indice fini $G\left(k_{v_{0}}\right)^{0} \subset G\left(k_{v_{0}}\right)$ et tout ouvert $\tilde{W} \subset(X \backslash F)\left(\mathbf{A}_{k}\right)$ satisfaisant $\tilde{W}^{\operatorname{Br}(X) \cap\left(A+B+f^{*} \operatorname{Br}_{G}(Z)\right)} \neq \emptyset$, il existe un $t \in Z^{\text {tor }}(k)$ tel que

$$
\operatorname{codim}\left(F \cap U_{t}, U_{t}\right) \geq 2 \text { et }\left(G\left(k_{v_{0}}\right)^{0} \cdot \tilde{W}\right) \cap\left(U_{t} \backslash\left(F \cap U_{t}\right)\right)\left(\mathbf{A}_{k}\right)^{B+A+f_{t}^{*} \operatorname{Br}_{G_{0}}\left(Z_{t}\right)} \neq \emptyset .
$$

Démonstration. Avec les constructions et notations de la démonstration du théorème 6.11, d'après (6.11), on a

$$
(X \backslash F)\left(\mathbf{A}_{k}\right)^{\operatorname{Br}(X) \cap\left(B+f^{*} \operatorname{Br}_{G}(Z)\right)}=p\left(\left(Y \backslash p^{-1} F\right)\left(\mathbf{A}_{k}\right)^{\operatorname{Br}(Y) \cap\left(p^{*} B+B_{1}+f_{V}^{*} \operatorname{Br}_{1}\left(T_{0} \times Z_{1}^{t o r}\right)\right)}\right) .
$$

Une application du corollaire 6.10 au triple (6.12) donne le résultat.

\section{LE RÉSULTAT PRINCIPAL}

Dans toute cette section, $k$ est un corps de nombres. Sauf mention explicite du contraire, une variété est une $k$-variété. Dans cette section, on établit le résultat principal : le théorème 7.6 (ou le théorème 7.5 sur la version de la fibration).

Rappelons la notion de sous-groupe de Brauer invariant (cf. Définition 3.1).

Soit $G$ un groupe linéaire connexe. Soit $S \subset \Omega_{k}$ un sous-ensemble fini. On considère tout sous-groupe ouvert d'indice fini $G\left(k_{S}\right)^{0}$ de $G\left(k_{S}\right)$. Ainsi $G\left(k_{S}\right)^{0}$ est fermé dans $G\left(k_{S}\right)$ et on a directement :

Lemme 7.1. Si $S=\infty_{k}$, alors $G\left(k_{\infty}\right)^{+} \subset G\left(k_{\infty}\right)$ est un sous-groupe ouvert d'indice fini et tout tel sous-groupe $G\left(k_{S}\right)^{0}$ contient $G\left(k_{\infty}\right)^{+}$.

Lemme 7.2. Soit $G$ un groupe linéaire connexe et simplement connexe. Soit $v \in \Omega_{k}$ une place. Supposons que $G$ est unipotent ou que $G$ est semi-simple et simple avec $G\left(k_{v}\right)$ non compact. Alors $G\left(k_{v}\right)$ ne possède pas de sous-groupe ouvert d'indice fini non-trivial.

Démonstration. Si $G \cong \mathbb{G}_{a}$, ceci vaut car $G\left(k_{v}\right) \cong k_{v}$ est uniquement divisible. Dans le cas où $G$ est unipotent, ceci vaut car il existe une filtration de $G$ de facteurs $\mathbb{G}_{a}$ ([Bo, Cor. 15.5 (ii)]). Ceci vaut aussi pour tout tel $G$ défini sur $k_{v}$.

Dans le cas où $G$ est semi-simple, simplement connexe et simple avec $G\left(k_{v}\right)$ non compact, si $v \in \infty_{k}$, ceci vaut par E. Cartan (cf. [PR, Prop. 7.6]). Si $v \notin \infty_{k}$, ceci vaut car $G\left(k_{v}\right)$ est engendré par les $k_{v}$-points des sous-groupes unipotents de $G$ sur $k_{v}$ (la conjecture de Kneser-Tits établie par Platonov, cf. [PR, Thm. 7.6]).

Proposition 7.3. Soit $G$ un groupe linéaire connexe et simplement connexe. Soit $S \subset \Omega_{k}$ un sous-ensemble fini non vide tel que $G^{\prime}\left(k_{S}\right)$ soit non compact pour chaque facteur simple $G^{\prime} d u$ groupe $G^{\text {sc }}$. Alors, pour tout sous-groupe ouvert d'indice fini $G\left(k_{S}\right)^{0} \subset G\left(k_{S}\right)$ et tout $G$-torseur $P$ sur $k$, l'ensemble $G\left(k_{S}\right)^{0} \cdot P(k)$ est dense dans $P\left(\mathbf{A}_{k}\right)$.

Démonstration. On peut supposer que $P\left(\mathbf{A}_{k}\right) \neq \emptyset$. Puisque $\operatorname{Br}_{a}(G)=0$, par le principe de Hasse pour un $G$-torseur (Kneser, Harder et Chernousov, cf. [Sko01, Thm. 5.1.1 (e)]), on a $P(k) \neq \emptyset$. Alors on peut supposer que $G \cong P$. 
Si $G$ est soit unipotent soit semi-simple, simplement connexe et simple, par hypothèse il existe une place $v \in S$ tel que $G\left(k_{v}\right)$ soit non compact. D'après le lemme $7.2, G\left(k_{v}\right)^{0}:=G\left(k_{S}\right)^{0} \cap G\left(k_{v}\right)$ est exactement $G\left(k_{v}\right)$. Une application de l'approximation forte de $G$ (Kneser, Platonov, cf. [PR, Thm. 7.12]) donne l'énoncé.

En général, le groupe $G$ possède une filtration de facteurs soit unipotents soit semi-simples simplement connexes et simples. Une application de la méthode de fibration ([CTX13, Prop. 3.1]) donne l'énoncé.

Proposition 7.4. Soient $G$ un groupe linéaire connexe, et $Z$ un $G$-espace homogène à stabilisateur géométrique connexe. Soit $S \subset \Omega_{k}$ un sous-ensemble fini non vide tel que $G^{\prime}\left(k_{S}\right)$ soit non compact pour chaque facteur simple $G^{\prime}$ du groupe $G^{s c}$. Supposons que $S=\infty_{k}$ ou que $\bar{k}[Z]^{\times}=\bar{k}^{\times}$. Alors, pour tout sous-groupe ouvert d'indice fini $G\left(k_{S}\right)^{0} \subset G\left(k_{S}\right)$ et tout ouvert $W \subset Z\left(\mathbf{A}_{k}\right)$ satisfaisant $W^{\mathrm{Br}_{G}(Z)} \neq \emptyset$, on a $Z(k) \cap\left(G\left(k_{S}\right)^{0} \cdot W\right) \neq \emptyset$.

Démonstration. Le cas où $S=\infty_{k}$ a été établi par Borovoi et Demarche ([BD, Thm. 1.4]). Ici, on donne une démonstration unifiée des deux cas considérés.

Une application du principe de Hasse pour un espace homogène à stabilisateur géométrique connexe (Borovoi [B96], cf. [Sko01, Thm. 5.2.1 (a)]) montrer que $Z(k) \neq \emptyset$. Il induit un $G$ morphisme $\pi: G \rightarrow Z$ tel que $Z \cong G / G_{0}$ avec $G_{0} \subset G$ un sous-groupe fermé connexe.

Par la résolution flasque [CT08, Porp. 5.4], il existe un groupe linéaire connexe $H$ et un homomorphisme surjectif $H \stackrel{\psi}{\rightarrow} G$ tels que $\operatorname{Ker}(\psi)$ soit un tore et $H$ soit quasi-trivial, i.e. $H^{\text {tor }}$ soit quasi-trivial et $H^{s c}=H^{s s}$. Alors $Z$ est une $H$-espace homogène à stabilisateur géométrique connexe, $H^{s c} \cong G^{s c}$ et, d'après le corollaire 3.11 et la proposition 3.9, on a : $\operatorname{Br}_{G}(Z)=\operatorname{Br}_{H}(Z)$. Le sous-groupe $H\left(k_{S}\right)^{0}:=\psi^{-1}\left(G\left(k_{S}\right)^{0}\right) \subset H\left(k_{S}\right)$ est ouvert d'indice fini. Alors on peut remplacer $G$ par $H$ et supposer que $G$ est quasi-trivial.

Notons $G \stackrel{\phi}{\rightarrow} G^{\text {tor }}$ le quotient torique maximal. Alors $\phi$ est lisse à fibres géométriquement intègres et donc $G\left(\mathbf{A}_{k}\right) \rightarrow G^{\text {tor }}\left(\mathbf{A}_{k}\right)$ est ouvert ([Cod, Thm. 4.5]). D'après le corollaire 5.13, il existe un ouvert $W_{1} \subset G\left(\mathbf{A}_{k}\right)$ et un point $z \in Z(k)$ tels que $\pi\left(W_{1}\right) \cdot z \subset W$ et $W_{1}^{\operatorname{Br}_{1}(G)} \neq \emptyset$. Puisque $G^{\text {tor }}$ satisfait l'approximation forte par rapport à $\operatorname{Br}_{1}\left(G^{t o r}\right)$ hors de $\infty_{k}$ (Harari [Ha08, Thm. 2]), il existe

$$
t \in G^{\text {tor }}(k) \cap\left(G^{\text {tor }}\left(k_{\infty}\right)^{+} \cdot \phi\left(W_{1}\right)\right) .
$$

Notons $G_{t}$ la fibre de $\phi$ au-dessus de $t$ et $G^{s s u}\left(k_{S}\right)^{0}:=G\left(k_{S}\right)^{0} \cap G^{s s u}\left(k_{S}\right)$. Ainsi $G_{t}$ est un $G^{s s u}$-torseur. D'après la proposition 7.3, l'ensemble $G^{s s u}\left(k_{S}\right)^{0} \cdot G_{t}(k)$ est dense dans $G^{s s u}\left(\mathbf{A}_{k}\right)$.

Dans le cas où $S=\infty_{k}$, puisque l'homomorphisme $G\left(k_{\infty}\right)^{+} \rightarrow G^{\text {tor }}\left(k_{\infty}\right)^{+}$est surjectif, il existe

$$
a \in G_{t}\left(\mathbf{A}_{k}\right) \cap\left(G\left(k_{\infty}\right)^{+} \cdot W_{1}\right) \quad \text { et donc } \quad g \in G_{t}(k) \cap\left(G^{s s u}\left(k_{S}\right)^{0} \cdot G\left(k_{\infty}\right)^{+} \cdot W_{1}\right) .
$$

Par le lemme 7.1, on a $G^{s s u}\left(k_{S}\right)^{0} \cdot G\left(k_{\infty}\right)^{+} \subset G\left(k_{S}\right)^{0}$ et donc $g \cdot z \in Z(k) \cap\left(G\left(k_{S}\right)^{0} \cdot W\right)$.

Dans le cas où $\bar{k}[Z]^{\times}=\bar{k}^{\times}$, par la suite exacte de Sansuc [S, Prop. 6.10], G $G_{0}^{\text {tor }} \rightarrow G^{\text {tor }}$ est surjectif et donc $G_{0} \rightarrow G \rightarrow G^{\text {tor }}$ est surjectif. Ainsi $G_{0}\left(k_{\infty}\right)^{+} \rightarrow G^{\text {tor }}\left(k_{\infty}\right)^{+}$est surjectif. Alors il existe $a \in G_{t}\left(\mathbf{A}_{k}\right) \cap\left(G_{0}\left(k_{\infty}\right)^{+} \cdot W_{1}\right)$ et donc

$$
g \in G_{t}(k) \cap\left(G^{s s u}\left(k_{S}\right)^{0} \cdot G_{0}\left(k_{\infty}\right)^{+} \cdot W_{1}\right) .
$$

Ainsi $g \cdot z \in Z(k) \cap\left(G\left(k_{S}\right)^{0} \cdot W\right)$. 
Théorème 7.5. Soient $G$ un groupe linéaire connexe, $G_{0} \subset G$ un sous-groupe fermé connexe et $Z:=G / G_{0}$. Soient $X$ une $G$-variété lisse géométriquement intègre, $U \subset X$ un $G$-ouvert et $U \stackrel{f}{\rightarrow} Z$ un $G$-morphisme. Soient $A \subset \operatorname{Br}(X)$ et $B \subset \operatorname{Br}_{G}(U)$ (cf. (3.1)) deux sous-groupes finis. Soit $S \subset \Omega_{k}$ un sous-ensemble fini non vide tel que $G^{\prime}\left(k_{S}\right)$ soit non compact pour chaque facteur simple $G^{\prime}$ du groupe $G^{s c}$. Supposons que $S=\infty_{k}$ ou que $\bar{k}[X]^{\times} / \bar{k}^{\times}=0$. Alors, pour tout sous-groupe ouvert d'indice fini $G\left(k_{S}\right)^{0} \subset G\left(k_{S}\right)$ et tout ouvert $W \subset X\left(\mathbf{A}_{k}\right)$ satisfaisant $W^{\operatorname{Br}(X) \cap\left(A+B+f^{*} \operatorname{Br}_{G}(Z)\right)} \neq \emptyset$, il existe un $z \in Z(k)$ de fibre $U_{z}$, tel que

$$
\left(G\left(k_{S}\right)^{0} \cdot W\right) \cap U_{z}\left(\mathbf{A}_{k}\right)^{B+A} \neq \emptyset .
$$

Démonstration. Le cas où $S=\infty_{k}$ découle du théorème 4.6 (2) et de la proposition 7.4.

Soit $\pi: Z \stackrel{\pi}{\rightarrow} T$ le quotient torique maximal de $Z$ et $G_{1} \subset G$ le stabilisateur de $G$ sur $T$ (cf. Définition 3.23). Pour tout $t \in T(k)$, notons $Z_{t}$ la fibre de $\pi$ au-dessus de $t$. Alors $Z_{t}$ est un $G_{1}$-espace homogène à stabilisateur géométrique connexe. Par la proposition 3.13, on a $\bar{k}\left[Z_{t}\right]^{\times} / \bar{k}^{\times}=0$.

Le cas où $\bar{k}[X]^{\times} / \bar{k}^{\times}=0$ découle du théorème 6.11 (2) et de la proposition 7.4.

Théorème 7.6. Soient $G$ un groupe linéaire connexe, $G_{0} \subset G$ un sous-groupe fermé connexe et $Z:=G / G_{0}$. Soient $X$ une $G$-variété lisse géométriquement intègre, $U \subset X$ un G-ouvert

et $U \stackrel{f}{\rightarrow} Z$ un $G$-morphisme. Soient $A \subset \operatorname{Br}(X)$ et $B \subset \operatorname{Br}_{G}(U)$ (cf. (3.1)) deux sous-groupes finis. Soit $S \subset \Omega_{k}$ un sous-ensemble fini non vide tel que $G^{\prime}\left(k_{S}\right)$ soit non compact pour chaque facteur simple $G^{\prime}$ du groupe $G^{\text {sc }}$.

(1) Si $S=\infty_{k}$ et, pour tout $z \in Z(k)$ de fibre $U_{z}$, l'ensemble $U_{z}(k)$ est dense dans $U_{z}\left(\mathbf{A}_{k}\right)_{\bullet}^{A+B}$, alors $X(k)$ est dense dans $X\left(\mathbf{A}_{k}\right) \bullet \operatorname{Br}(X) \cap\left(A+B+f * \operatorname{Br}_{G}(Z)\right)$.

(2) $S i \bar{k}[X]^{\times}=\bar{k}^{\times}$et, pour tout $z \in Z(k)$, la fibre $U_{z}$ satisfait l'approximation forte de BrauerManin par rapport ̀̀ $A+B$ hors de $S$, alors $X$ satisfait l'approximation forte de Brauer-Manin par rapport à $\operatorname{Br}(X) \cap\left(A+B+f^{*} \operatorname{Br}_{G}(Z)\right)$ hors de $S$.

Démonstration. Ceci suit immédiatement du théorème 7.5.

Remerciements. Je remercie très chaleureusement Jean-Louis Colliot-Thélène et Fei Xu pour plusieurs discussions. Je remercie également Cyril Demarche, Qifeng Li et Giancarlo Lucchini Arteche pour leurs commentaires. Projet soutenu par l'attribution d'une allocation de recherche Région Ile-de-France.

\section{RÉFÉRENCES}

[Bo] A. Borel : Linear algebraic groups, Second edition, Graduate Texts in Mathematics 126, Springer-Verlag, New York, 1991.

[B96] M. Borovoi : The Brauer-Manin obstruction to the Hasse principle for homogeneous spaces with connected or abelian stabilizer, J. reine angew. Math. 473 (1996), 181-194.

[BD] M. Borovoi et C. Demarche : Manin obstruction to strong approximation for homogeneous spaces, Comment. Math. Hev. 88 (2013), 1-54.

[Cod] B. Conrad : Weil and Grothendieck approaches to adelic points, Enseign. Math. 58 (2012), 61-97.

[CX] Y. Cao, F. Xu : Strong Approximation with Brauer-Manin Obstruction for Toric Varieties, arXiv : 1311.7655 (2013). 
[CX1] Y. Cao, F. Xu : Strong Approximation with Brauer-Manin Obstruction for Groupic Varieties, arXiv : $1507.04340(2015)$.

[CDX] Y. Cao, C. Demarche, F. Xu : Comparing descent obstruction and Brauer-Manin obstruction for open varieties, arxiv : 1604.02709 (2016).

[CT-Beijing] J-L. Colliot-Thélène : Lectures on linear algebraic groups, notes, april 2007, voir : http ://www.math.u-psud.fr/ colliot/BeijingLectures2Juin07.pdf.

[CT08] J-L. Colliot-Thélène : Résolutions flasques des groupes linéaires connexes, J. reine angew. Math. 618 (2008), 77-133.

[CTH] J-L. Colliot-Thélène et D. Harari : Approximation forte en famille, J. reine angew. Math. 710 (2016), 173-198.

[CTSa] J-L. Colliot-Thélène et J.-J. Sansuc : Principal homogeneous spaces under flasque tori, applications, Journal of Algebra 106 (1987) 148-205.

[CTSb] J-L. Colliot-Thélène et J.-J. Sansuc : La descente sur les variétés rationnelles, II, Duke Math. J. 54 (1987) 375-492.

[CTX09] J-L. Colliot-Thélène et F. Xu : Brauer-Manin obstruction for integral points of homogeneous spaces and representations by integral quadratic forms, Compos. Math. 145(2009) 309-363.

[CTX13] J-L. Colliot-Thélène, F. Xu : Strong approximation for the total space of certain quadric fibrations, Acta Arithmetica 157 (2013), 169-199.

[D11] C. Demarche : Le défaut d'approximation forte dans les groupes linéaires connexes, Proc.London Math. Soc. 102 (2011), 563-597.

[E] Ekedahl, T : An effective version of Hilbert's irreducibility theorem, in : Séminaire de théorie des nombres de Paris, 1988-1989, Progress Math. 91, Birkhäuser (1990), 241-248.

[Gro] A. Grothendieck : Le groupe de Brauer, I, II, III, in : Dix exposés sur la cohomologie des schémas, North Holland \& Masson 1968.

[Ha94] D. Harari : Méthode des fibrations et obstruction de Manin, Duke Math. J. 75 (1994), 221-260.

[Ha08] D. Harari : Le défaut d'approximation forte pour les groupes algébriques commutatifs, Algebra \& Number Theory 2 (5)(2008) 595-611.

[HS05] D. Harari and T. Szamuely. Arithmetic duality theorems for 1-motives, J. reine angew. Math. 578 (2005) 93-128.

[Mi80] J. S. Milne : Étale Cohomology, Princeton Math. Ser 33, Princeton University Press, Princeton 1980.

[PR] V. P. Platonov et A. S. Rapinchuk: Algebraic groups and number theory, Academic Press, 1994.

[S] J.-J. Sansuc: Groupe de Brauer et arithmétique des groupes algébriques linéaires sur un corps de nombres, J. reine angew Math. 327 (1981), 12-80.

[Se65] J.-P. Serre : Cohomologie Galoisienne, Lecture Notes in Mathematics, vol 5, Springer, Berlin, 1965.

[Sko90] A. N. Skorobogatov: On the fibration method for proving the Hasse principle and weak approximation, in : Séminaire de théorie des nombres de Paris, 1988-1989, Progress Math. 91, Birkhäuser (1990), 205-219.

[Sko01] A. N. Skorobogatov: Torsors and Rational Points, Cambridge Tracts in Mathematics, vol. 144, Cambridge University Press, 2001.

[Wei14] D, Wei, Strong approximation for the variety containing a torus, arxiv : 1403.1035 (2014).

[Wei16] D. Wei, Open descent and strong approximation, arXiv : 1604.00610 (2016).

YANG CAO

Laboratoire de Mathématiques D'Orsay

Univ. Paris-Sud, CNRS, Univ. Paris-Saclay

91405 Orsay, France

E-mail address: yang.cao@math.u-psud.fr 